\title{
CSI 2264: Simultaneous optical and X-ray variability in pre-main sequence stars
}

\section{Time resolved X-ray spectral analysis during optical dips and accretion bursts in stars with disks}

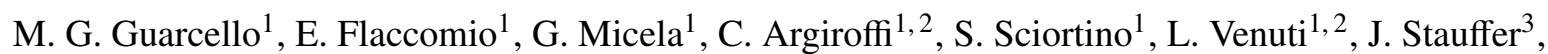 \\ L. Rebull ${ }^{3}$, and A. M. Cody ${ }^{4}$ \\ 1 INAF-Osservatorio Astronomico di Palermo, Piazza del Parlamento 1, 90134 Palermo, Italy \\ e-mail: mguarce@astropa.unipa.it \\ 2 Dip. di Fisica e Chimica, Università di Palermo, Piazza del Parlamento 1, 90134 Palermo, Italy \\ 3 Spitzer Science Center, California Institute of Technology, Pasadena, CA 91125, USA \\ 4 NASA Ames Research Center, Kepler Science Office, Mountain View, CA 94035, USA
}

Received 31 October 2016 / Accepted 13 January 2017

\begin{abstract}
Context. Pre-main sequence stars are variable sources. The main mechanisms responsible for their variability are variable extinction, unsteady accretion, and rotational modulation of both hot and dark photospheric spots and X-ray-active regions. In stars with disks, this variability is related to the morphology of the inner circumstellar region $(\leq 0.1 \mathrm{AU})$ and that of the photosphere and corona, all impossible to be spatially resolved with present-day techniques. This has been the main motivation for the Coordinated Synoptic Investigation of NGC 2264, a set of simultaneous observations of NGC 2264 with 15 different telescopes.

Aims. In this paper, we focus on the stars with disks. We analyze the X-ray spectral properties extracted during optical bursts and dips in order to unveil the nature of these phenomena. Stars without disks are studied in a companion paper.

Methods. We analyze simultaneous CoRoT and Chandra/ACIS-I observations to search for coherent optical and X-ray flux variability in stars with disks. Then, stars are analyzed in two different samples. In stars with variable extinction, we look for a simultaneous increase of optical extinction and X-ray absorption during the optical dips; in stars with accretion bursts, we search for soft X-ray emission and increasing X-ray absorption during the bursts.

Results. We find evidence for coherent optical and X-ray flux variability among the stars with variable extinction. In 9 of the 24 stars with optical dips, we observe a simultaneous increase of X-ray absorption and optical extinction. In seven dips, it is possible to calculate the $N_{\mathrm{H}} / A_{V}$ ratio in order to infer the composition of the obscuring material. In 5 of the 20 stars with optical accretion bursts, we observe increasing soft X-ray emission during the bursts that we associate to the emission of accreting gas. It is not surprising that these properties are not observed in all the stars with dips and bursts, since favorable geometric configurations are required.

Conclusions. The observed variable absorption during the dips is mainly due to dust-free material in accretion streams. In stars with accretion bursts, we observe, on average, a larger soft X-ray spectral component not observed in non-accreting stars.
\end{abstract}

Key words. circumstellar matter - stars: pre-main sequence - stars: rotation - stars: variables: T Tauri, Herbig Ae/Be

\section{Introduction}

Pre-main sequence (PMS) stars can be classified according to their spectral energy distribution (SED) in the infrared (Lada 1987); the youngest PMS stars still surrounded by both a contracting envelope and a circumstellar disk are classified as Class I objects. Class II objects are PMS stars surrounded by circumstellar disks, whose envelope is partially or completely dissipated. Class III sources are PMS stars whose disks have been dissipated or at least evolved into debris disks. The intermediate phases of stars with pre-transition disks (with an intermediate gap separating inner and outer disks, Espaillat et al. 2007) and transition disks (with cleared inner regions, Muzerolle et al. 2010) have been more recently added to this evolutionary scenario. The evolution and dissipation of circumstellar disks involve several physical processes, such as gas accretion onto the central star driven by viscosity and mediated by the magnetic field (e.g.,
Koenigl 1991), photoevaporation (e.g., Störzer \& Hollenbach 1999), dust aggregation and settling (e.g. Testi et al. 2014), and environmental feedback (e.g., Guarcello et al. 2016), with timescales which range from a few thousands to approximately $10^{7}$ yr (Haisch et al. 2001; Hernández et al. 2007; Mamajek 2009).

The disk inner region $(\leq 0.1 \mathrm{AU})$ is very important for the accretion process, the magnetic coupling between disk and central star, and the evolution of the entire disk and the star itself. This region is, however, very difficult to analyze, even in the systems close to our Sun and even with infrared and radio interferometry. The analysis of the SEDs of disk-bearing stars has achieved many successes in this direction, such as the discovery of the inner disk wall and pre-transitional gaps, however the physical parameters derived from SED fitting are model-dependent and likely affected by the strong intrinsic variability of these sources in the optical and infrared. A unique insight into the 
very innermost region of circumstellar disks could be provided by studying the simultaneous variability in optical, infrared and even X-rays, which are intimately connected with the morphology and properties of the circumstellar environment.

Optical variability of PMS stars has been the subject of several studies (e.g., Joy 1945; Alencar et al. 2010; Morales-Calderón et al. 2011; Wolk et al. 2013; Cody et al. 2014), and originally it was one of the criteria used by Joy (1945) to identify the newly discovered class of T Tauri stars. Herbst et al. (1994) presented the first classification of variability of young stars based on their light curves. Type $\mathrm{I}^{1}$ light curves are periodic and often sinusoidal, resulting from the rotational modulation of cold photospheric spots. Type II light curves are less periodic and interpreted as the result of variable veiling continuum and rotational modulation of an accretion hot spot. Type III light curves vary irregularly because of variable extinction. A more detailed classification of light curves of disk-bearing stars has recently been proposed by Cody et al. (2014), as part of the CSI 2264 project described below. They classify "burster" light curves as those characterized by rapid (0.1-1 day) and symmetric increments of flux (the symmetric shape of the burst means that its rising part is not impulsive and it is similar to the decaying phase, which distinguishes them from flares); "dipper" light curves showing transient fading events (dips); "periodic" and "quasi-periodic" light curves resulting from rotational modulation; "stochastic" light curves which, even if not dominated by bursts or dips, are nevertheless characterized by brightness changes over a variety of timescales; and "long-time variables" with monotonic light variations (either brightening of fading) over timescales of days and weeks.

The different types of optical and infrared light curves of stars with disks reflect the different morphology of the inner disks and probe the geometry of the accretion process. Following the classification of Cody et al. (2014), "dipper" stars are AA Tau analogs. AA Tau is a well-studied variable star with a disk characterized by recurrent occultation of the central star by warps in the circumstellar disk (Bouvier et al. 1999, 2003, 2007; Ménard et al. 2003; Grosso et al. 2007) located close to the co-rotation radius (Rucinski et al. 2008; Alencar et al. 2010; Cody \& Hillenbrand 2010). These warps in the inner disks are, in general, due to misalignment between the rotation and magnetic axes, and they are located at the base of steady accretion streams, which are stable over several stellar rotation periods (Alencar et al. 2010). Alencar et al. (2010) have also shown that AA Tau like variability is common in stars with inner disks; in their study of the optical and infrared variability of the stars in NGC 2264, they have found that nearly $40 \%$ of the stars with inner disks are characterized by AA Tau-like variability.

Also, accretion contributes to variable optical and infrared extinction (e.g., McGinnis et al. 2015). The accretion streams are dust free, but small amounts of dust can be trapped at the base of the streams and survive until the temperature is higher than the sublimation temperature. As suggested by Stauffer et al. (2015), the dust particles trapped in the accretion streams can be responsible for small dips in the optical light curves. Another way accretion may contribute to optical variability is by the emergence of the optical emission from accretion hot spots on stellar surface (Stauffer et al. 2014). In fact, the accreting material funneled by the magnetic field falls onto the stellar surface with a velocity of several hundreds of km per second. This energy is released at the accretion shock as soft X-ray, UV, and optical radiation.

\footnotetext{
1 The type of the light curves must not be confused with the class used to classify YSOs.
}

Whether or not it is possible to observe variability in X-rays due to the accretion process and variable circumstellar extinction remains unknown. PMS stars are very bright X-ray sources (Feigelson \& Decampli 1981), with their X-ray emission exceeding that of main-sequence (MS) stars with the same mass by three or four orders of magnitude (e.g., Montmerle 1996). The main component of this X-ray emission is the quiescent emission, or at least apparently quiescent since the "quiescent" coronal emission can be actually the result of a superposition of small flares (e.g., Caramazza et al. 2007), from a scaled-up version of the solar corona with plasma at $10-30 \mathrm{MK}$, powered and confined by a dynamo-generated stellar magnetic field (e.g., Feigelson \& Kriss 1981). However, the lack of an evident MS-like rotation-activity relation in PMS stars, together with the large and so far unexplained scatter of X-ray brightness, indicate that the emission mechanism might be more complicated than this. Intense flaring activity produced by magnetic reconnection is observed in PMS stars (e.g., Flaccomio et al. 2003), and they can be so powerful as to require non-solar geometry for the stellar magnetic field (Jardine et al. 2006). Sometimes, flares in PMS stars are modeled with very large loops that may even reach the surface of the inner disks (Favata et al. 2005). Accretion also contributes to the emission of soft X-rays (e.g., Kastner et al. 2002), produced in the accretion shocks and observed in a few Class II stars, such as TW Hya and BP Tau, using detailed spectroscopic analysis (Kastner et al. 2002; Stelzer \& Schmitt 2004; Schmitt et al. 2005; Argiroffi et al. 2011; Curran et al. 2011). However, this X-ray emission has only been unambiguously identified in the nearest stars with disks, primarily because it is difficult to distinguish from the coronal soft X-ray emission. Additionally, a significant part of this emission is likely absorbed by accreting and circumstellar material itself (Argiroffi et al. 2011; Bonito et al. 2014).

$\mathrm{X}$-ray emission from PMS stars is strongly variable over a large range of timescales and amplitudes. The most evident source of X-ray variability is undoubtedly flares. The rise phase is much shorter than the decline phase (which can last several hours), and the peak flux can be approximately 100 times the quiescent flux (Favata et al. 2005). There are, however, other sources of X-ray variability. Stellar coronae are not homogeneous, and their X-ray emission can be modulated by stellar rotation (Flaccomio et al. 2005). This has been observed in the Chandra Orion Ultradeep Project (COUP, Getman et al. 2005): an approximately 13-day continuous Chandra/ACIS-I observation of the Orion Nebula Cluster. Also accretion spots are not uniformly distributed over the stellar surface, resulting in a rotational modulation of soft X-ray emission. This, however, has only been observed in the T Tauri star V4046 Sgr (Argiroffi et al. 2012). Variable absorption of the coronal emission by circumstellar and accreting material can be another source of X-ray variability (e.g., Flaccomio et al. 2010).

Simultaneous optical, infrared, and X-ray variability in stars with disks can be the consequence of unsteady accretion, variable extinction, and rotational modulation (Flaccomio et al. 2012). Stassun et al. (2007) find no convincing evidence for coherent optical and X-ray flux variability in the PMS stars in Orion. No evidence of coherent X-ray and infrared variability in PMS stars is found by Flaherty et al. (2014) in their study of the PMS stars in IC 348, concluding that X-rays are not an important source of heating for the circumstellar material. A different result has been obtained by Flaccomio et al. (2010) in their study of the PMS stars in NGC 2264. They find a significant correlation between optical and X-ray flux variability using two $30 \mathrm{ks}$ Chandra/ACIS-I observations (separated by 16 days) and 
simultaneous CoRoT data. This correlation is only observed in Class II sources, and is not observed in the hard X-ray band. This is interpreted as a consequence of variable absorption of both photospheric and coronal emission.

In this paper, we analyze new simultaneous X-ray and optical observations of NGC 2264, obtained as a part of the CSI 2264 project, to search for connections between optical and X-ray variability in PMS stars with disks observed in the quiescent emission. We show the effectiveness of time-resolved X-ray spectral analysis in stars with disks using the optical light curves as template to isolate interesting features such as accretion bursts and optical dips. NGC 2264, the object of this study, and the CSI 2264 project are described in Sect. 2. In Sect. 3, we describe the CoRoT and Chandra data sets analyzed and the selection of the targets; in Sect. 4, we present evidence for coherent optical and X-ray flux variability. In Sect. 5, we present a detailed analysis of the variability of the X-ray properties during dips and bursts observed in the CoRoT light curves. Results are summarized and discussed in Sect. 6.

\section{NGC 2264 and the CSI 2264 project}

The study of the variability of young stars provides an opportunity to probe the very inner circumstellar region $(\leq 0.1 \mathrm{AU})$ and the morphology of stellar coronae and photospheres. This is one of the main motivations of the Coordinated Synoptic Investigation of NGC 2264 (CSI 2264, Cody et al. 2014; Stauffer et al. 2014). This project is a unique and unprecedented cooperative project involving simultaneous observations of NGC 2264 with 15 ground and space telescopes, covering the electromagnetic spectrum from X-rays to mid-infrared. The entire list of the observations that are part of the CSI 2264 project can be found in Cody et al. (2014). The main optical photometric dataset is obtained from observations with the Convection, Rotation and Planetary Transits satellite (CoRoT, Baglin et al. 2006) from December 1st 2011 to January 3rd 2012, using the second CCD designed for exoplanet studies. CoRoT observed an area of $1.3 \times 1.3$ square degrees centered on NGC 2264 with a cadence of $512 \mathrm{~s}$, or $32 \mathrm{~s}$ for the brighter sources.

NGC 2264 is the only young cluster (1-5 Myr, Rebull et al. 2002; Dahm 2008) falling in one of the CoRoT eyes (i.e., the two regions with a $10^{\circ}$ diameter close to the galactic center and anticenter observed with CoRoT), making it a unique target for monitoring the variability of young stars using this telescope. This cluster is relatively nearby ( $760 \mathrm{pc}$, Park et al. 2000), and part of the local spiral arm. It is characterized by non-uniform extinction across the field, with both richly populated low-extinction regions containing approximately two thousand sources in total, and highly embedded regions where the star-formation process is still ongoing, as evidenced by the presence of stars with thick disks, molecular outflows, and Herbig-Haro objects (Teixeira et al. 2012). The median extinction of known cluster members is relatively low $\left(A_{V} \sim 0.45^{m}\right.$, Rebull et al. 2002). The cluster population is well defined, and it includes a few early type stars, such as the O7V star S Monocerotis (Schwartz et al. 1985 ) and approximately a dozen B-type stars. NGC 2264 is the only cluster within one kpc of the Sun besides the Orion Nebula Cluster with such a large mass spectrum. Figure 1 shows a DSS2 image of the central region of NGC 2264. The field of view of the Chandra observations analyzed here is indicated. The actual CoRoT field, $1.3^{\circ} \times 1.3^{\circ}$ wide, is larger than the field shown in Fig. 1.

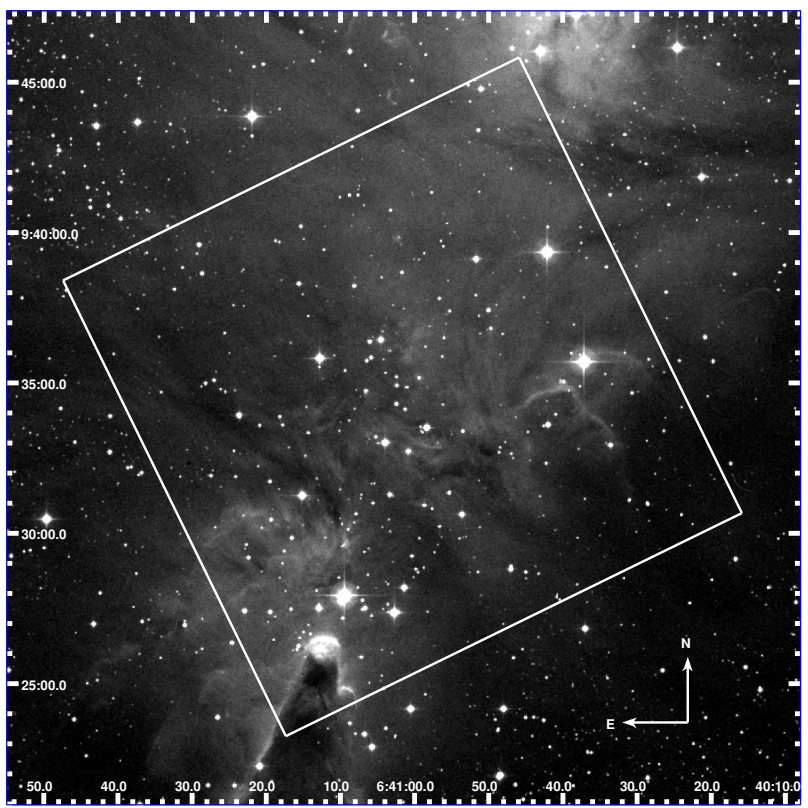

Fig. 1. DSS-2 image of the central region of NGC 2264, with the field observed with Chandra/ACIS-I marked.

\section{Data analysis and targets selection}

\subsection{CoRot light curves}

CoRoT photometry is only available for stars falling in preselected pixel masks. For this reason, the list of targets was carefully pre-compiled before the observations, and it includes 1617 known candidate members of NGC 2264 and 2129 field stars falling in the area of the cluster (Cody et al. 2014). The membership criteria used for target selection are described in Cody et al. (2014) and they are based on: 1) optical photometry compatible with the cluster in color-magnitude diagrams as defined by Flaccomio et al. (2006); 2) strong $\mathrm{H} \alpha$ emission (Rebull et al. 2002; Lamm et al. 2004; Sung et al. 2008); 3) X-ray detection (Ramírez et al. 2004; Flaccomio et al. 2006); 4) radial velocity compatible with NGC 2264 (Fúrész et al. 2006); and 5) presence of a circumstellar disk (Sung et al. 2009; Cody et al. 2014). Only targets with $11^{m}>R>17^{m}$ were observed.

Light curves were produced after correction from gain and zero offset, jitter, electromagnetic interference, and background subtraction, following the standard CoRoT data reduction pipeline (Samadi et al. 2006). Data from hot pixels and outliers were flagged and removed from the light curves. While most of the light curves refer to the full CoRoT band, for a subset of stars three light curves are provided, referring to "red", "green", and "blue" bands. However, since these bands are not calibrated, in this work we only use the sum of the three bands, that is, the white light curves. CoRoT light curves are not in an absolute magnitude scale since the photometric zero point of the CoRoT data varies between runs. A photometric zero point of $26.74^{m}$ specific for the CSI 2264 CoRoT observations has been obtained by Cody et al. (2014) after comparing the mean CoRoT flux of selected stars with their available $R$-band photometry (from Rebull et al. 2002; Lamm et al. 2004; Sung et al. 2008). Flaccomio et al. (in prep.) obtained a slightly smaller zero point $\left(26.6^{m}\right)$, which is the value adopted in this paper.

A number of systematic errors are not corrected by the standard pipeline and affect CoRoT light curves. The most important 
Table 1. Chandra/ACIS-I observations log.

\begin{tabular}{ccc}
\hline \hline Obs.ID & Exposure (ks) & Date \\
\hline 14368 & 74.44 & Dec. 3rd 2011 \\
13610 & 92.54 & Dec. 5th 2011 \\
13611 & 60.23 & Dec. 7th 2011 \\
14369 & 66.16 & Dec. 11th 2011 \\
\hline
\end{tabular}

systematic error, affecting approximately $10 \%$ of the observed light curves, consists of abrupt jumps in flux that are due to rapid changes in detector temperature. In this paper, we do not attempt any correction for this effect and we reject, when necessary, CoRoT data that are affected by such jumps. We use only CoRoT data which are not flagged as suspicious data points.

\subsection{X-ray data}

In this paper, we use the data obtained from four Chandra/ACISI observations taken from December 3rd to December 11th 2011 (P.I. G. Micela) during the CoRoT run. Table 1 shows the log of these observations. The total nominal exposure is $300 \mathrm{ks}$, and all the observations are pointed at $\alpha_{\mathrm{J} 2000}=06: 40: 58.70$, $\delta_{\mathrm{J} 2000}=+09: 34: 14$, with almost coincident roll angles. In order to make astrometry compatible with 2MASS, each image has been reprojected by matching bright 2MASS sources detected in X-rays and correcting for the observed offsets both in RA and Dec.

A detailed analysis of the Chandra/ACIS-I (Weisskopf et al. 2002; Garmire et al. 2003) observations available for NGC 2264, including source detection, photon extraction and spectral fitting, is presented in Flaccomio et al. (in prep.). Briefly, all events were fully reprocessed using the CIAO task chandrarepro. Sources were detected using the wavelet-based algorithm PWDetect (Damiani et al. 1997), adopting a significance threshold of 4.4, roughly resulting in approximately ten expected spurious detections. Event extraction, source repositioning, and validation were performed with the IDL software ACIS Extract (AE, Broos et al. 2010). AE is capable of: i) defining the extraction region around each source, accounting for crowding and the shape of the PSF at different off-axis angles; ii) extracting both source and background events, the latter in a suitable region around the source; and iii) compiling photometry, calculating light curves, and providing source statistics. After excluding candidate spurious detections, a total of 694 X-ray sources were validated. X-ray spectra were fitted using Xspec v.12.8.1 (Arnaud 1996). Observed spectra were rebinned in photon energy in order to have a signal-to-noise ratio larger than one in each bin.

\subsection{Targets selection}

The main objective of this paper is to analyze the simultaneous optical and X-rays variability of stars with disks in NGC 2264.

We adopt the selection of stars with disks presented in Sung et al. (2009) and Cody et al. (2014), which is based on the slope of the SEDs in the IRAC and MIPS $24.0 \mu \mathrm{m}$ bands and suitable color-color diagrams where the typical loci populated by disk-bearing stars can be defined. Among the 95 candidate stars with disks observed with CoRoT and falling in the ACISI field, 86 are detected in X-rays. We also define a subsample of 79 stars with disks (75 detected in X-rays) which are actively accreting. These stars are selected using two criteria: $\mathrm{H} \alpha$ equivalent width (EW) larger than $10 \AA$ (Rebull et al. 2002) or using the $r^{\prime}-i^{\prime}$ versus $r^{\prime}-\mathrm{H} \alpha$ color-color diagram from the INT (Isaac Newton Telescope, $2.5 \mathrm{~m}$ ) Photometric $\mathrm{H} \alpha$ Survey (IPHAS; Drew et al. 2008). In this color-color diagram, in fact, it is possible to select candidate accreting stars as those with red $r^{\prime}-\mathrm{H} \alpha$ color, and derive an estimate of the $\mathrm{H} \alpha \mathrm{EW}$ from this color (Barentsen et al. 2011). Disk-bearing sources without signatures of accretion are identified as stars with passive disks (15 stars, 10 detected in X-rays). We also selected 10 candidate stars with transition disks (all detected in X-rays) as those showing excesses only at $8.0 \mu \mathrm{m}$ and $24 \mu \mathrm{m}$. The infrared excesses in each infrared band are calculated using the $Q_{V I J A}$ color indices similar to those defined in Guarcello et al. $(2009,2013)$. These color indices compare the $V-I$ and $J-A$ colors, with $A$ being $K$ or one of the Spitzer bands. Since these indices increase as $J-A$ becomes more red, and they are independent from extinction, they can be used to separate the extinguished stellar population from that with intrinsic red colors, and to calculate the excess in each infrared band. We refer to Damiani et al. (2006) and Guarcello et al. (2009) for a detailed description of these color indices and their use.

We also adopt the classification of the CoRoT light curves provided by Cody et al. (2014): "Bursters" (13\% of the stars with disks in NGC 2264 observed with CoRoT); sources with variable extinction ("dippers", $21.5 \%$, approximately half of which are periodic); stars with light curves showing stochastic behavior (13\%, named "stochastic"); non-variable stars (19\%); stars with periodic or quasi-periodic variability $(21 \%)$; long-term variables (1\%); eclipsing binaries (1\%); and sources with unclassified variability $(11 \%)$. Since the classification of the "stochastic" stars may vary according to different time windows, we reviewed the behavior of these sources in the period simultaneous with the four ACIS-I observations. Sometimes we consider stars as "dippers" or "bursters" depending on the dominant phenomenon occurring during the Chandra observations.

\section{Coherent optical and X-ray flux variability}

In this section we analyze the simultaneous flux variability in optical and X-rays. Coherent flux variability is expected to occur when: i) the star is affected by variable extinction due to circumstellar material simultaneously obscuring both stellar photosphere and coronal active regions; or ii) photospheric spots, accretion hot spots, and coronal active regions simultaneously emerging during stellar rotation.

\subsection{Existing studies}

A search for coherent optical and X-ray flux variability in T Tauri stars has only been attempted in a small number of cases, given the paucity of existing simultaneous optical and X-ray observations of young clusters. Stassun et al. (2006) studied BVRI data of the Orion Nebula taken with the WIYN 0.9 m telescope at the Kitt Peak National Observatory (KPNO) and the $1.5 \mathrm{~m}$ Cassini telescope in Loiano, Italy, simultaneous with the COUP observations. These optical data have been taken with a cadence of one data point per hour, not comparable to the excellent time resolution of the CoRoT data. These authors find evidence of coherent optical and X-ray flux variability in approximately $5 \%$ of the analyzed T Tauri stars. This result has been interpreted in terms of rotational modulated emission from accretion spots, and the lack of correlation in most of the observed stars is taken as evidence that X-ray emission arises mainly from the stellar corona rather than from the accretion spots distributed over the stellar surface. 
M. G. Guarcello et al.: CSI 2264: Simultaneous optical and X-ray variability in pre-main sequence stars
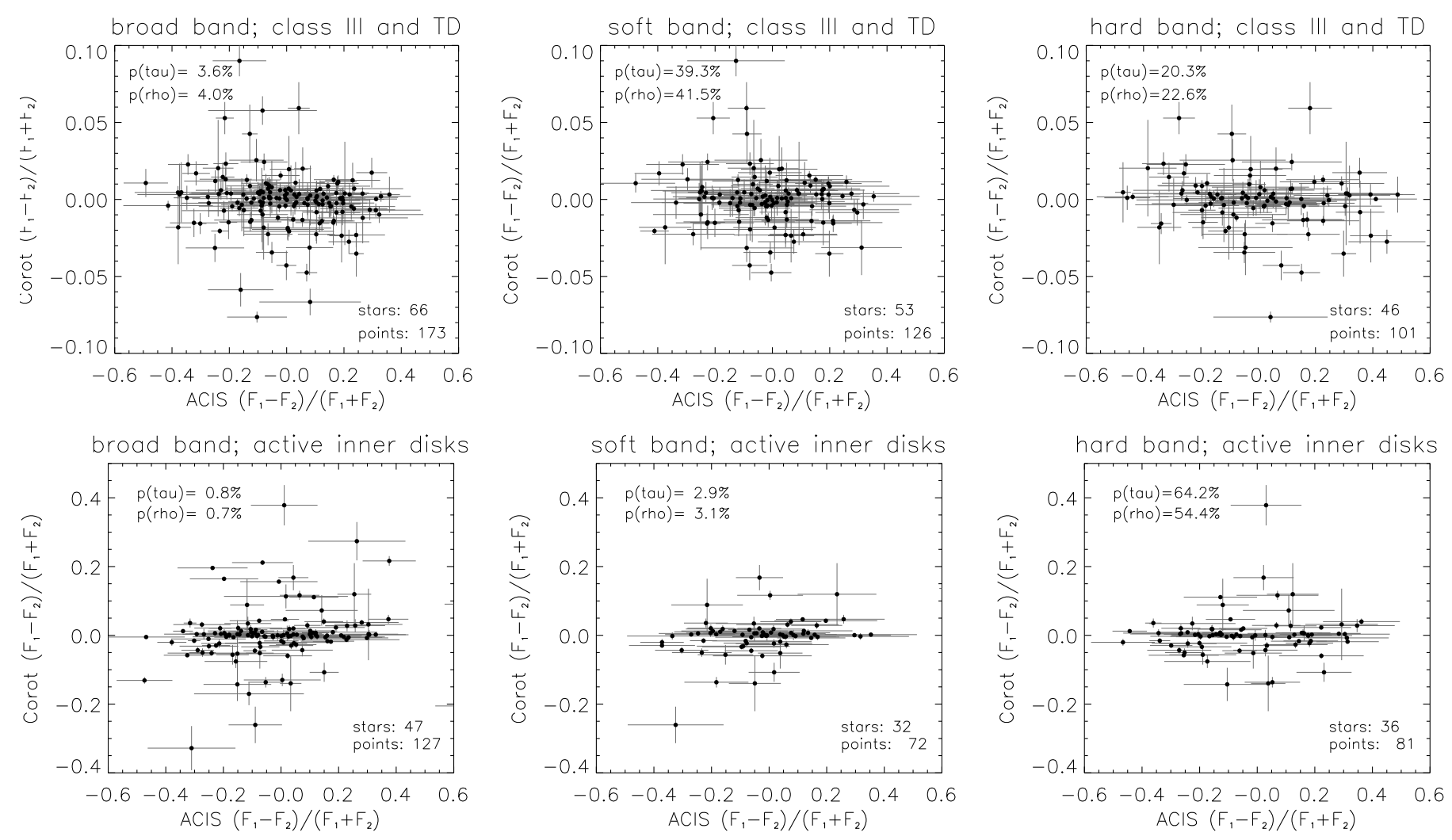

Fig. 2. Comparison between the optical and X-rays flux variability, calculated using Eq. (1), shown separately for the stars with transition disks and Class III objects (upper panels) and stars with inner disks, both passive and accreting (lower panels). Note the different $y$-axis scaling for the top and bottom rows. X-ray photons are selected in the broad-band $(0.5-8 \mathrm{keV}$; left panels $)$, soft band $(0.5-1.5 \mathrm{keV}$; central panels), and hard band $(1.5-8 \mathrm{keV}$; right panels). In each panel, the null-hypothesis probabilities of no correlation resulting from the Spearman $(\rho)$ and Kendall $(\tau)$ rank correlation test are shown. Each point corresponds to the variability observed between two consecutive given Chandra frames for each source. The total number of stars and points used in each plot is also indicated.

Flaccomio et al. (2010) search for correlations between optical and X-ray flux variability in the young stars in NGC 2264 by comparing the variation of the X-ray fluxes observed in two 30 ks Chandra/ACIS-I observations (Obs. IDs: 9768 and 9769; P.I. G. Micela) with that of the CoRoT "white" fluxes from simultaneous observations. They find evidence of correlated flux variability in a sample of 24 low-mass T Tauri stars with disks, and only in the soft $0.5-1.5 \mathrm{keV} \mathrm{X}$-ray band. This is interpreted as evidence that the correlation is a result of time-variable absorption by the surrounding circumstellar material. These authors also suggest that the obscuring material is dust-depleted, as expected from accretion streams covering part of the stellar corona and photosphere.

\subsection{Coherent optical and $X$-ray flux variability in stars with disks of NGC 2264}

The starting point for our search of coherent optical and X-ray flux variability in the NGC $2264 \mathrm{~T}$ Tauri stars was the result obtained by Flaccomio et al. (2010). We first replicated their approach to verify whether our new data confirm their finding. To this aim, we selected all the X-ray sources observed with CoRoT with more than ten counts detected in at least two Chandra observations, discarding known massive and intermediate-mass stars (i.e., rejecting stars more massive than $2 M_{\odot}$ ). Hereafter, we will refer to the time intervals corresponding to the Chandra observations listed in Table 1,that is where both CoRoT and X-ray data are available, as Chandra frames. For each selected source, we calculated the mean X-ray photon flux and CoRoT whiteband flux observed in each Chandra frame where no flares are detected. Flares are automatically detected using the approach defined in Caramazza et al. (2007), i.e., dividing the X-ray light curve in blocks of almost constant count rate (Maximum Likelihood Blocks) and classifying these intervals according to the measured count-rates and its time derivative (a detailed analysis of the flares observed in NGC 2264 will be presented in Flaccomio et al., in prep.). We then calculate the flux variability among two Chandra frames $n$ and $m$ as:

$\Delta_{\text {flux }}=\frac{F_{n}-F_{m}}{F_{n}+F_{m}}$

where $F_{n}$ is the optical or the X-ray photon flux detected during the $n$th Chandra frame.

The result of this approach is shown in Fig. 2, where each point compares the value of $\Delta_{\text {flux }}$ calculated for a given source in two consecutive Chandra frames. Error bars are propagated from the uncertainties in X-ray and optical fluxes, the former computed with Poissonian statistics, the latter from the RMS of the optical light curve observed during the given Chandra frames. We use X-ray data in different energy bands (broad in the left panel, soft in the central, and hard in the right). In Fig. 2, we consider the sample of stars with an inner disk, both passive or accreting, and that of stars with a transition disk and Class III objects (whose variability is expected to be similar, Cody et al. 2014) separately. The classification of Class III objects and the analysis of their variability is the subject of a companion paper. 
The amplitude of the observed variability is different in these two samples of stars. We observe a smaller amplitude of optical variability in stars without close circumstellar material than in those with inner disks, with the difference of a factor of between two and four. Conversely, the range of variability in X-rays is similar in the two cases. This is due to the fact that rotational modulation of photospheric spots and active regions, typical of the inner-disk-free sample, results in smaller amplitude modulation than, for instance, variable extinction (Venuti et al. 2015). In each panel, we also show the results of correlation tests, which, in general, indicate that there is no obvious correlation between broad-band X-ray and optical measurements for stars with disks, though some of the extreme points (obtained from 17 stars) drive a statistically significant correlation in the X-ray broad energy band in the stars with inner disks. This is not observed in the stars without close circumstellar material.

Using the classification of optical light curves provided by Cody et al. (2014), we obtain deeper insight into the different mechanisms responsible for coherent optical and X-ray flux variability. In Fig. 3, we investigate correlations between optical and $\mathrm{X}$-ray flux variability for those stars with circumstellar disks whose light curves are classified by Cody et al. (2014). The Xrays count-rates are indicated separately for the broad-band (upper panel), soft band (central panel), and hard band (bottom panel). It is evident that the stars with large amplitude optical variability, dominating any possible correlation, are "dipper" or "stochastic" stars. The results of the correlation tests are shown for the entire sample and for the "stochastic"+"dippers" sample (values inside the brackets), and they indicate that a correlation between optical and X-ray flux variability is possible only in stars with disks and variable extinction. This is reinforced by the fact that the "stochastic" stars with large variability in optical in Fig. 3 have optical dips during the Chandra frames.

\section{Variability of the X-ray properties during optical dips and bursts}

\subsection{Time-resolved analysis of stellar X-ray properties}

The unique data set analyzed in this work allows us to study in detail how the X-ray properties of disk-bearing stars vary during events observed in optical, specifically dips due to variable extinction and bursts due to accretion. Among the 86 stars with disks observed both with CoRoT and Chandra, 51 are bright enough in X-rays to allow a reliable analysis of their variability. Among them, 24 show well-defined flux dips in the CoRoT data, and 20 show accretion burst signatures, with some stars showing both properties.

To this aim, we divided each Chandra time frame into smaller time intervals, defined in order to isolate interesting features in the CoRoT light curves, such as dips and bursts. For each time interval, we then calculated the mean CoRoT flux and extract the X-ray photons detected during the time interval to calculate the corresponding X-ray photon flux $F_{\mathrm{X}}$ (in units of photons $\mathrm{cm}^{-2} \mathrm{~s}^{-1}$ ), the hydrogen column density $N_{\mathrm{H}}$ (in units of $10^{22} \mathrm{~cm}^{-2}$ ), the temperature of the emitting plasma $k T$ (in $\mathrm{keV}$ ), and the $10 \%, 25 \%$, and $50 \%$ photon energy quantiles $\left(E_{10 \%}\right.$, $E_{25 \%}, E_{50 \%}$, respectively, in $\mathrm{keV}$ ). Flaccomio et al. (in prep.) demonstrate that $E_{10 \%}$ and $E_{25 \%}$ are well correlated with the hydrogen column density obtained by fitting the observed X-ray spectra of NGC 2264 low-mass members with 1T or 2T thermal plasma models, thus being useful probes for the X-ray absorption affecting the stars. Some of the time intervals are narrow with few photons observed, but despite the small signal-to-noise
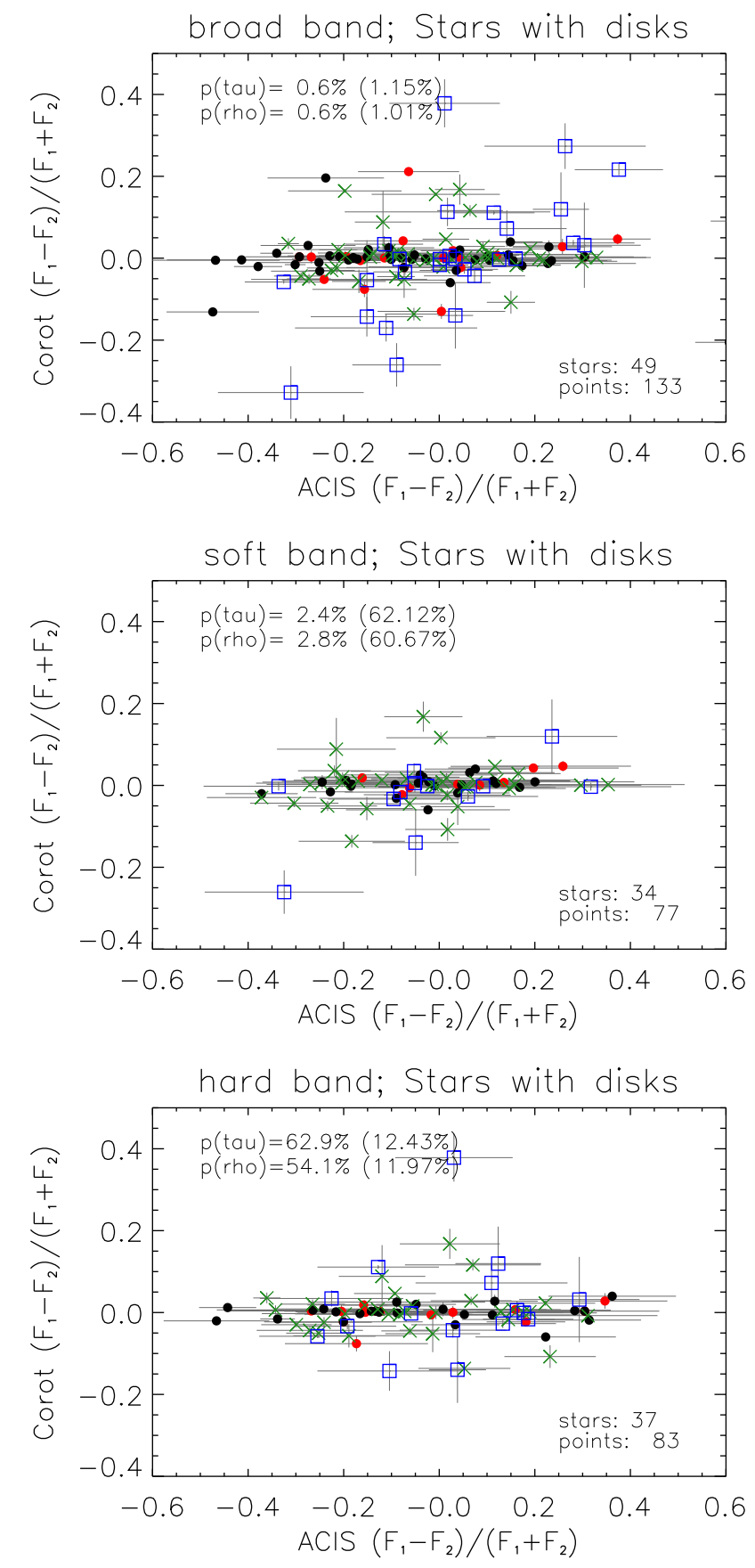

Fig. 3. Comparison between the flux variability in optical and X-rays as in Fig. 2, for disk-bearing stars whose optical variability has been classified by Cody et al. (2014). "Burster", "unclassified", "not variable", "quasi-periodic", "long term variable", and "multi-periodic" stars are marked with filled dots (red for the "bursters" and black for the remainder), while "stochastic" stars are marked with green $\times$ symbols and "dipper" stars with blue squares. In the upper left corner of each panel we show the probabilities derived from the correlation tests using both the entire sample and the dippers+stochastic stars (the latter inside brackets).

ratio, such small time intervals are necessary to isolate those features that we want to analyze.

The X-ray properties in each time interval were calculated fitting the observed X-ray spectra with $1 \mathrm{~T}$ and 2T APEC ionization-equilibrium plasma isothermal model (Smith et al. 2001), assuming the sub-solar elements abundance defined by 
Table 2. $\mathrm{H} \alpha$ EW from Dahm \& Simon (2005) and $\dot{M}_{\text {acc }}$ from Venuti et al. (2014) of the stars discussed in Sects. 5.3 and 5.4.

\begin{tabular}{cccc}
\hline \hline Mon-ID & H $\alpha E W$ & H $\alpha$ shape & $\dot{M}_{\mathrm{acc}}$ \\
\hline & $\AA$ & & $M_{\odot} / \mathrm{yr}$ \\
\hline 456 & 13.1 & asymmetric & \\
1167 & 23.4 & & $4.6 \times 10^{-9}$ \\
412 & 30.7 & asymmetric & $\sim 10^{-7}$ \\
717 & 24.5 & & $2.8 \times 10^{-8}$ \\
619 & 94.3 & & $4 \times 10^{-8}$ \\
491 & 67.2 & & $3 \times 10^{-8}$ \\
774 & 14.3 & & \\
1076 & 2.6 & & $1.7 \times 10^{-8}$ \\
808 & 50.2 & asymmetric & \\
370 & 113.2 & & $1.7 \times 10^{-9}$ \\
326 & 27.9 & & \\
474 & 104.7 & & \\
357 & 8 & & \\
945 & 66.3 & asymmetric & \\
771 & 28.9 & & \\
765 & 18.2 & & \\
103 & 6.4 & & \\
378 & 8.5 & & \\
\hline
\end{tabular}

Maggio et al. (2007), and affected by photoelectric absorption from both interstellar and circumstellar material treated using the TBABS model (Wilms et al. 2000). Best-fit models were chosen with the C-statistic and the quality of the fit is tested using the Xspec tool goodness. The limit for acceptable fits in this paper was set to null-hypothesis probability of a good fit $\left(P_{\%}\right)$ equal to $5 \%$. The significance of the parameters obtained with the spectral fit was tested by the analysis of the confidence contours in the C-stat space with the Xspec tool steppar.

\subsection{Disks properties}

We analyzed the SEDs of some stars using the online SED fitting tool presented by Robitaille et al. $(2007)^{2}$. With this tool it is possible to compare the observed SEDs with a set of YSO models covering an extensive parameter space, 20000 models, each at ten different inclination angles, for a total of 200000 distinct SEDs. For the fit, we constrain the source distances to that of NGC $2264(760 \mathrm{pc})$ and explore a wide range of possible extinctions (from $A_{V}=0.1^{m}$ to $A_{V}=100^{m}$ ). The best-fit models are those that satisfy the condition: $\chi^{2}-\chi_{\text {best }}^{2} \leq 3$, where $\chi_{\text {best }}^{2}$ is the reduced chi-square of the best-fit model (as suggested by Robitaille et al. 2007). Accretion rates and $\mathrm{H} \alpha \mathrm{EW}$ of the stars discussed in this section are taken from Dahm \& Simon (2005) and Venuti et al. (2014), and shown in Table 2.

\subsection{Optical dips with increasing $X$-ray absorption}

In this section, we analyze the variability of those stars with disks with increasing X-ray absorption during the optical dips, that is where optical extinction and X-ray absorption may increase simultaneously. For each of these stars, we show the CoRoT light curve, with the Chandra frames and the time intervals that we defined, together with the variability of some of the following X-ray quantities: $N_{\mathrm{H}}$ in units of $10^{22} \mathrm{~cm}^{-2}, \mathrm{kT}$, and $E_{10 \%}$ and

\footnotetext{
2 Now available at https://sedfitter.readthedocs.org/en/ stable/
}

$E_{25 \%}$ in keV. The X-ray spectra observed during the time intervals together with the best fit models are shown in Appendix A; Appendix D contains the entire CoRoT light curves of all the stars analyzed in this paper. Each star is labeled with both the CoRoT ID and the Mon- ID.

Mon-456: Mon-456 (Fig. 4) is an interesting case of simultaneous optical and X-ray variability. This Class II K4 star, with a rotation period of 5.05 days, is moderately accreting and it is classified as a "quasi periodic dipper" by Cody et al. (2014) and as a "AA Tau like" star by McGinnis et al. (2015) according to both their 2008 and 2011 data. The latter authors also observe increasing reddening of the CoRoT light curve during the dips, typical of AA Tau (Bouvier et al. 2003), and that several flux dips appear in the light curve during each rotation period, suggesting the presence of various secondary accretion streams. Large optical dips are observed during three Chandra frames (left panel in Fig. 4):

- A deep optical dip is observed in part during the time interval \#1.

- The intervals \#2 and \#4 are dominated by X-ray flares.

- The time intervals \#5 and \#6 are characterized by two optical dips with similar X-ray properties (right panels in Fig. 4).

- Time interval \#3 has a rising optical light curve.

The time variability of $N_{\mathrm{H}}$ (shown in Fig. 4) and the timeresolved X-ray spectra (Fig. A.1) suggest a larger $N_{\mathrm{H}}$ during the dips in \#5 and \#6 than in the other time intervals, but in each dip the X-ray absorption is not well constrained. In order to better constrain the X-ray absorption during the dips, we fitted the average X-ray spectrum over the summed time interval \#5+\#6 and compare the results with those obtained from time intervals $\# 2+\# 3+\# 4$. From the latter spectrum, we obtain $N_{\mathrm{H}}=0_{0}^{+0.11} \times 10^{22} \mathrm{~cm}^{-2}\left(P_{\%}=0.68\right)$, while for the former $N_{\mathrm{H}}=0.46_{-0.27}^{+0.32} \times 10^{22} \mathrm{~cm}^{-2}\left(P_{\%}=0.85\right)$, suggesting a larger hydrogen column density during the optical dips. The $>2 \sigma$ significance of this difference is confirmed by the contours in the C-stat space shown in Fig. 4.

There is no evidence for larger $N_{\mathrm{H}}$ during the dip in \#1, and the X-ray spectral fit of the spectrum observed in \#1+\#5+\#6 results in a poorly constrained fit $\left(P_{\%}=0.002\right)$. However, some evidence for the X-ray spectrum getting harder during this dip (i.e. increasing $E_{10 \%}$ ) is presented in Appendix B.

Mon-1167: Fig. 5 shows the optical and X-ray variability of the M3 disk-bearing star Mon-1167, classified as a "quasiperiodic stochastic" by Cody et al. (2014) and as an aperiodic extinction dominated star during 2008 and an "AA Tau analog" during 2011 by McGinnis et al. (2015). This star is moderately accreting and its light curve is characterized by:

- Small optical dips in the time intervals \#5, \#8, and \#11, together with a large optical dip observed only partially during the time interval \#3.

- Optical emission rising during \#1 and \#2, then steeply falling in \#10.

- No peculiar features are observed in \#4, \#6, \#7, \#9, \#12.

During the dips, the CoRoT flux decreases by $5.5 \%$ (\#3), $1.9 \%$ (\#5), 2.1\% (\#8), and 1.3\% (\#11). The obtained $N_{\mathrm{H}}$ is only significantly different from zero only during \#3 and \#8, although in \#8, the error bar is large and marginally compatible with the value observed in \#7 (right bottom panels in Fig. 5).

The C-stat contours from the spectral fits in the time intervals \#3 and \#8 support the evidence of a larger $N_{\mathrm{H}}$ during \#3, while a possible solution with $N_{\mathrm{H}}=0$ within $68 \%$ confidence is possible during the time interval \#8. 

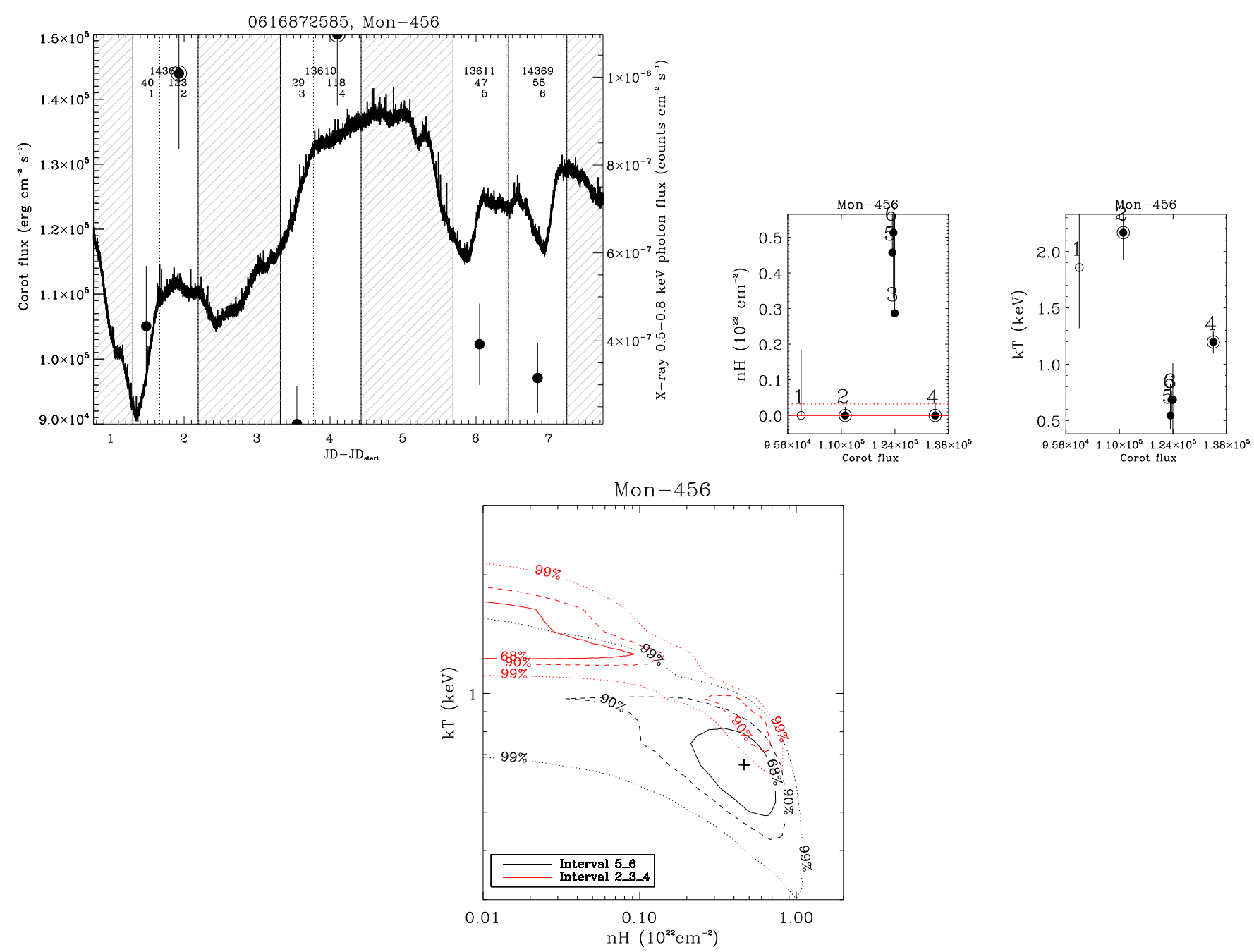

Fig. 4. Optical and X-ray variability of Mon-456. The upper left panel shows part of the CoRoT light curve. The Chandra frames are the unshaded intervals (the shaded intervals are those not observed with Chandra). The dotted vertical lines delimit the time intervals we defined; the black dots mark the observed X-ray photon fluxes in the broad-band (circled if, during the given time interval, the source flared in X-rays). The numbers above the light curve indicate the Chandra Obs.ID of the given Chandra frame (top number), the number of detected X-ray photons (middle number), and the label (bottom number) of the given time interval. The upper right panels show the variability of selected X-ray properties (in this case, the hydrogen column density $N_{\mathrm{H}}$ in units of $10^{22} \mathrm{~cm}^{-2}$; and the plasma temperature $k T$, in keV) versus the median CoRoT fluxes observed in the time intervals indicated by the labels. Empty circles mark intervals with unacceptable X-ray spectral fit. The bottom panel shows contour levels in the C-stat space enclosing solutions within 68\% (solid contour), 90\% (dashed contour), and 99\% (dotted contour) statistical confidence from the X-ray spectral fit of Mon-456 during the intervals \#2+\#3+\#4 (red contours) and \#5+\#6 (black contours). The crosses indicate the values obtained from the best fit in the latter case.

Mon-412: Fig. 6 shows the optical and X-ray variability of the accreting M1 Class II object Mon-412, classified as a "burster" by Cody et al. (2014) and Stauffer et al. (2014). The CoRoT light curve of Mon-412 may have been contaminated by a nearby disk-free cluster member more than one magnitude fainter in $I$ band and falling in the CoRoT mask. CoRoT light curve (upper left panel) is characterized by accretion bursts and dips:

- Small optical burst-like features observed in \#1, \#2, \#3, \#8.

- Prominent optical burst-like features observed in part during \#4 and \#9.

- In \#5, \#6, and \#7 the optical light curve shows a sequence of dip-like and/or burst-like features, with the CoRoT flux being $3.5 \%$ lower in \#6 than in \#5 and \#7.

It is not clear from the CoRoT light curve alone whether the variability observed in the third Chandra frame is due to two optical bursts in \#5 and \#7 or to an optical dip in \#6. Some hint is provided by the variability of the X-ray properties (shown in the right panels). During the time interval \#6, in fact, $N_{\mathrm{H}}$ varies from 0 to $1.2_{0.24}^{0.38} \times 10^{22} \mathrm{~cm}^{-2}$ together with $E_{10 \%}, E_{25 \%}$, and $E_{50 \%}$ (the latter not shown in Fig. 6) reaching the highest values observed in this star. However, as shown in Fig. 6, the 68\% statistical confidence region in the C-stat space for the interval \#6 also allows solutions at low $N_{\mathrm{H}}$. We conclude then that there are hints of a larger X-ray absorption during \#6, but this is not significantly supported by the time-resolved X-ray spectral analysis.

Mon-717: Mon-717 shows a large optical dip (see Fig. 7), longer than the third Chandra frame and with the CoRoT flux decaying by $25.3 \%$ (corresponding to an increase (ignoring the size of the obscuring feature with respect to the stellar disk) of $A_{V}$ by $0.38^{m}$ ) and a full width at half medium (FWHM) of 0.6 days. This star is a Class II M0.5 star that is moderately accreting. The source is faint in X-rays, with only 10 photons detected during 

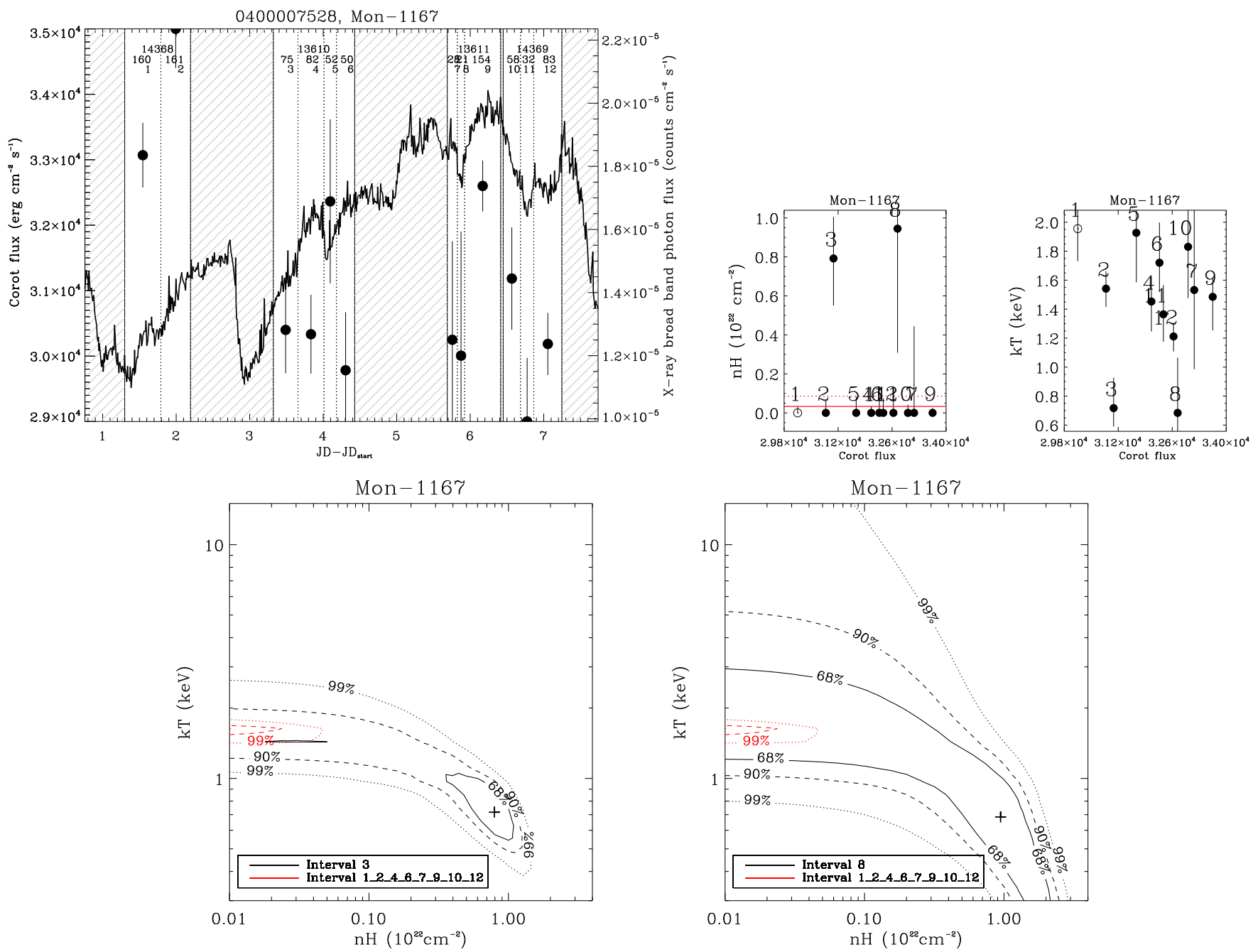

Fig. 5. Optical and X-ray variability of Mon-1167. Panel format and content are as in Fig. 4. The contours are shown for the X-ray spectral fit of the spectrum observed during the intervals \#3 (black contours, bottom left panel) and \#8 (black contours, bottom right panel). In both bottom

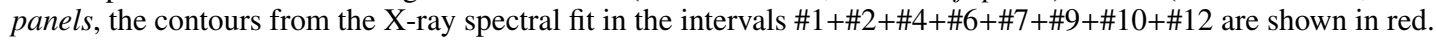

the dip and 20 in the previous Chandra frame. The X-ray emission is, however, harder during the dip than in the other frames, as suggested by the variability of the photon energy quantiles (Fig. 7), suggesting that not only the optical emission but also the X-ray emission is more absorbed during the third Chandra frame.

Mon-119: the optical and X-ray variability of Mon-119 (spectral type K6, Dahm \& Simon 2005), classified as a "stochastic" star by Cody et al. (2014) and as a star whose variability is mostly driven by variable accretion by Stauffer et al. (2016), is shown in Fig. 8. The CoRoT light curve shows several features:

- The optical emission rises by a factor 1.12 during \#1, followed by a more quiescent phase (\#2).

- An intense optical burst occurring between the first and second Chandra frames, whose final part is observed in the time interval \#3 (with only 19 X-ray photons detected).

- Two optical dips are observed in the time intervals \#4 and \#6 separated by a quiescent phase (\#5).

- The optical emission is higher during the third Chandra frame with at least two evident bursts (intervals \#7 and \#9), separated by a more quiescent phase (\#8).
- The fourth Chandra frame (\#10) is dominated by an intense $\mathrm{X}$-ray and optical flare.

In the two dips (\#4 and \#6), the CoRoT flux decreases by $8.9 \%$ and $15.7 \%$ with respect to the optical emission observed during the time interval \#5. As shown in the top right panels in Fig. 8, excluding the flare, $N_{\mathrm{H}}$ is always compatible with zero in all the time intervals except in the second Chandra frame (\#4, and \#6). In order to verify whether a significantly larger $N_{\mathrm{H}}$ is observed during these two optical dips, we fit the $1 \mathrm{~T}$ thermal plasma model to the average X-ray spectrum summing the time intervals \#4+\#6. The average value of $N_{\mathrm{H}}$ suggested by the best fit model is $N_{\mathrm{H}} \# 4+\# 6=0.21_{-0.16}^{+0.23} \times 10^{22} \mathrm{~cm}^{-2}$, which is significantly larger than zero (and larger than the absorption observed in the remaining intervals) within a $68 \%$ confidence range. This is confirmed by the contours in the C-stat space (Fig. 8).

Mon-619: Mon-619 is a K8.5V star actively accreting from its disk, and both Cody et al. (2014) and McGinnis et al. (2015) classified this star as an aperiodic extinction-dominated star. The CoRoT light curve of Mon-619 in Fig. 9, shows:

- A large optical dip that dominates the second Chandra frame (\#2).

- Two smaller optical dips or an accretion burst occurring during a large dip during \#1. 

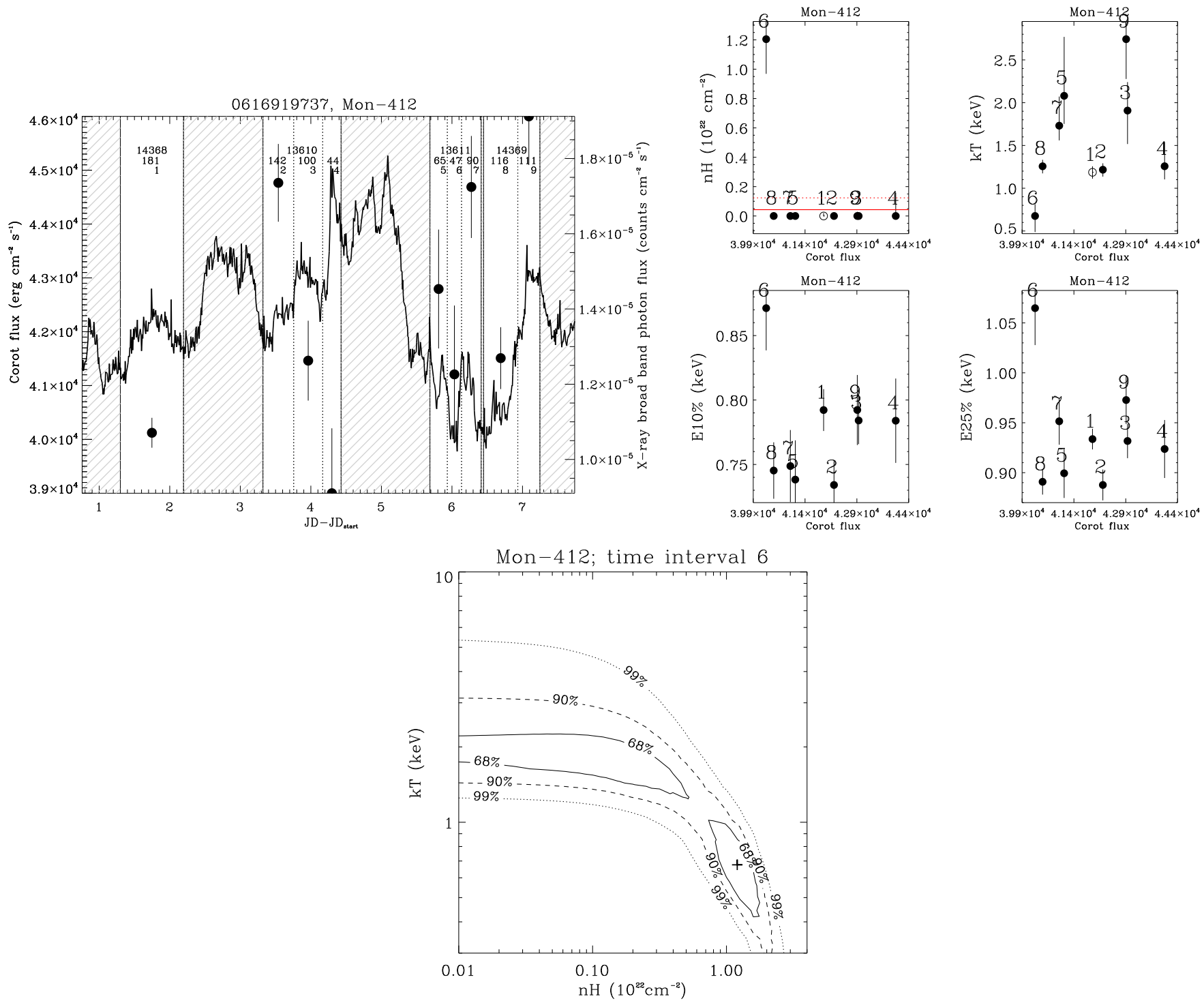

Fig. 6. Optical and X-ray variability of Mon-412. Panel format and content generally follows Fig. 4. In this case, we show the hydrogen column density $N_{\mathrm{H}}$ in units of $10^{22} \mathrm{~cm}^{-2}$, the plasma temperature $k T$ and the $10 \%$ and $25 \%$ photon energy quantiles in keV, and contour levels in the C-stat space. The contours are from the X-ray spectral fit of the spectrum observed during the interval \#6.
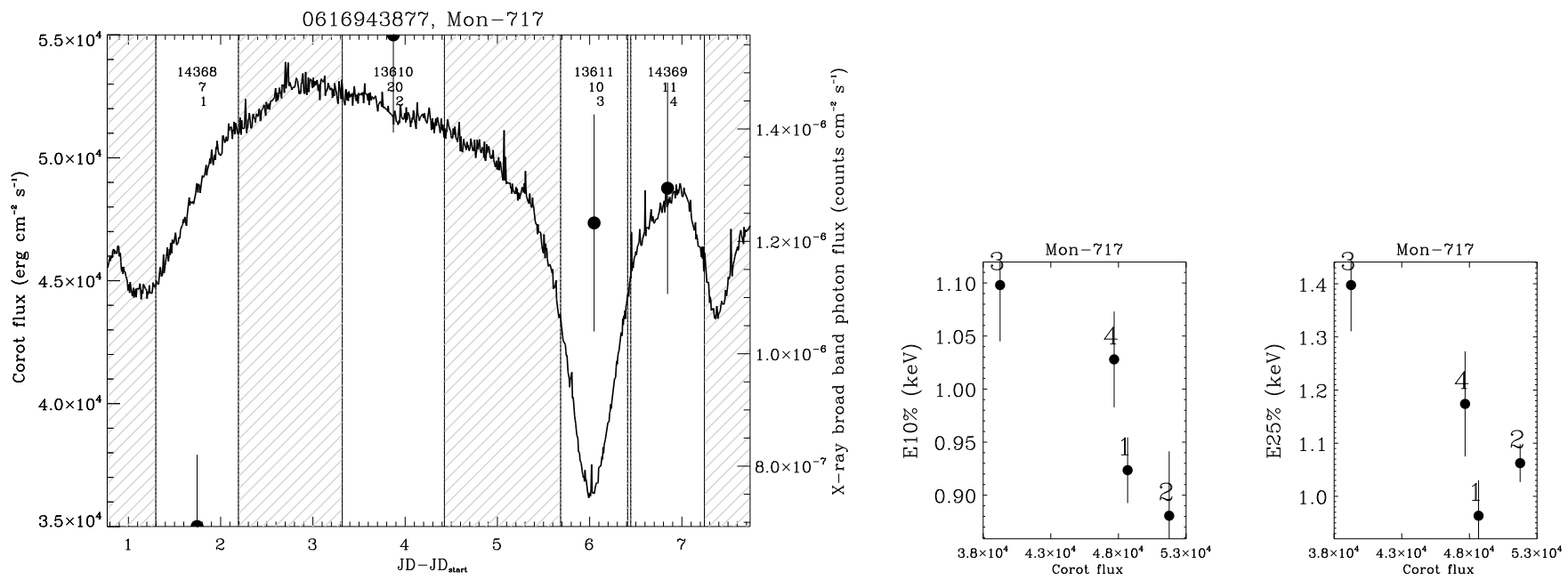

Fig. 7. Optical and X-ray variability of Mon-717. Panel format and content generally follows Fig. 4. In this case, the $10 \%$ and $25 \%$ photon energy quantiles in $\mathrm{keV}$ are shown. 
M. G. Guarcello et al.: CSI 2264: Simultaneous optical and X-ray variability in pre-main sequence stars
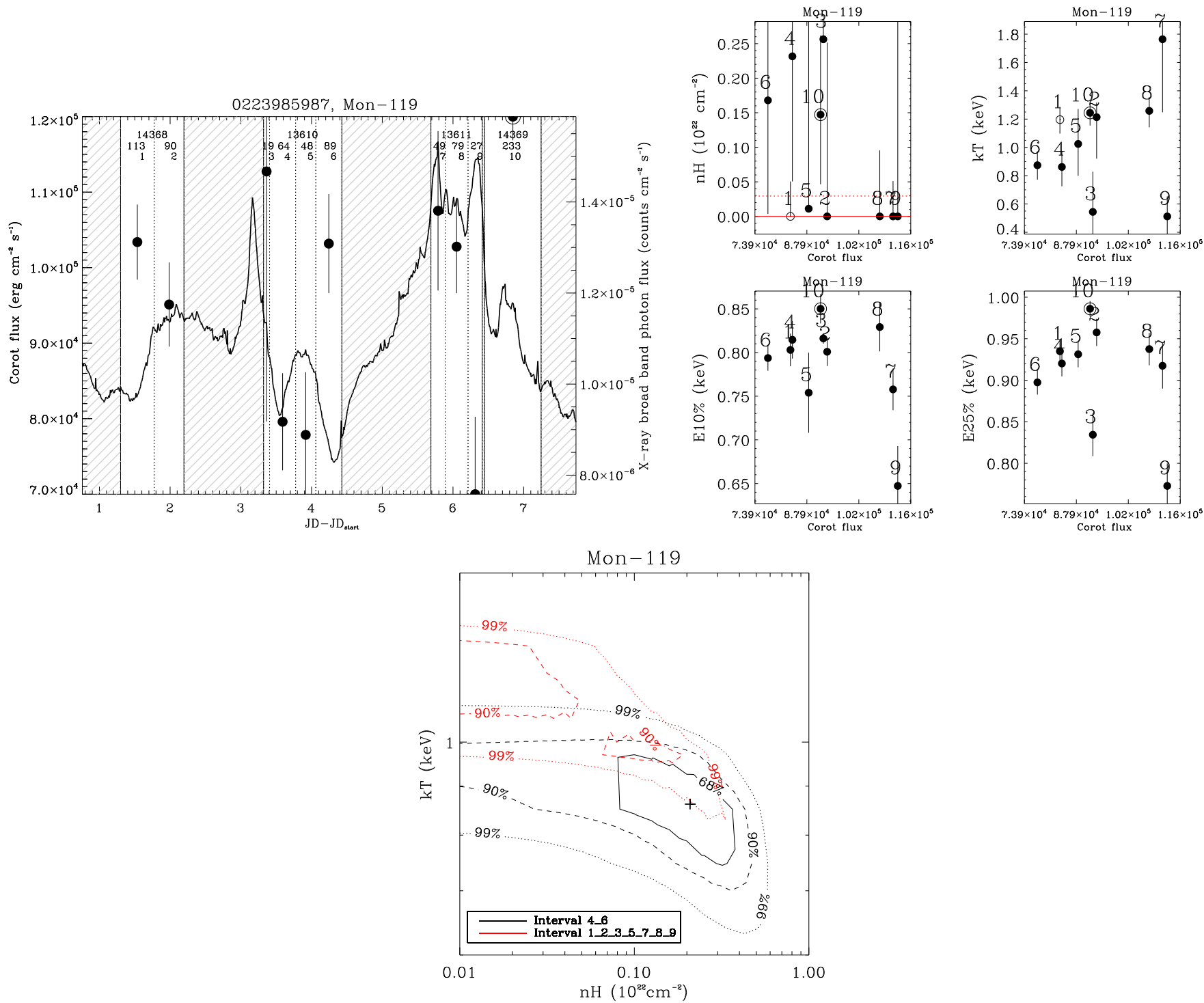

Fig. 8. Optical and X-ray variability of Mon-119, with panel format and content generally following Fig. 4. The contours are from the X-ray

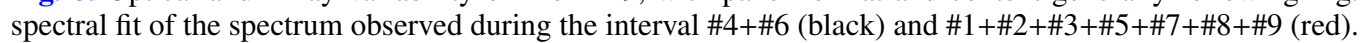
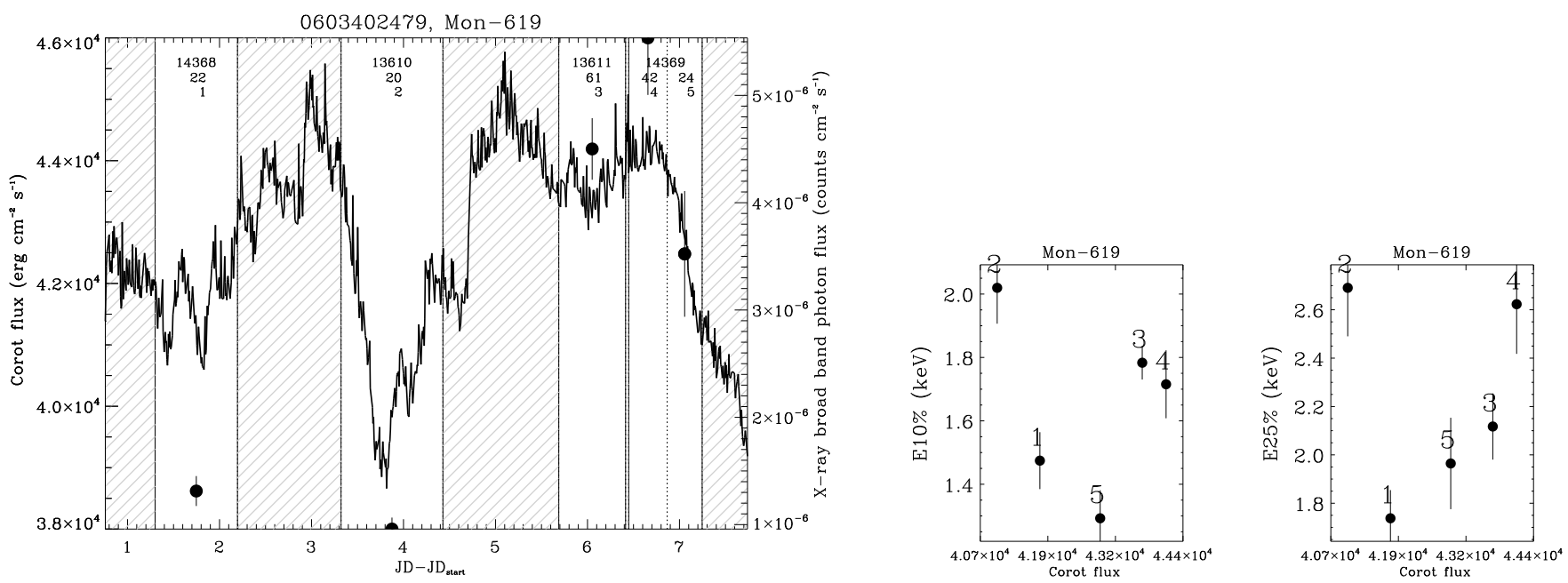

Fig. 9. Optical and X-ray variability of Mon-619, with panel format and content generally following Fig. 4. 

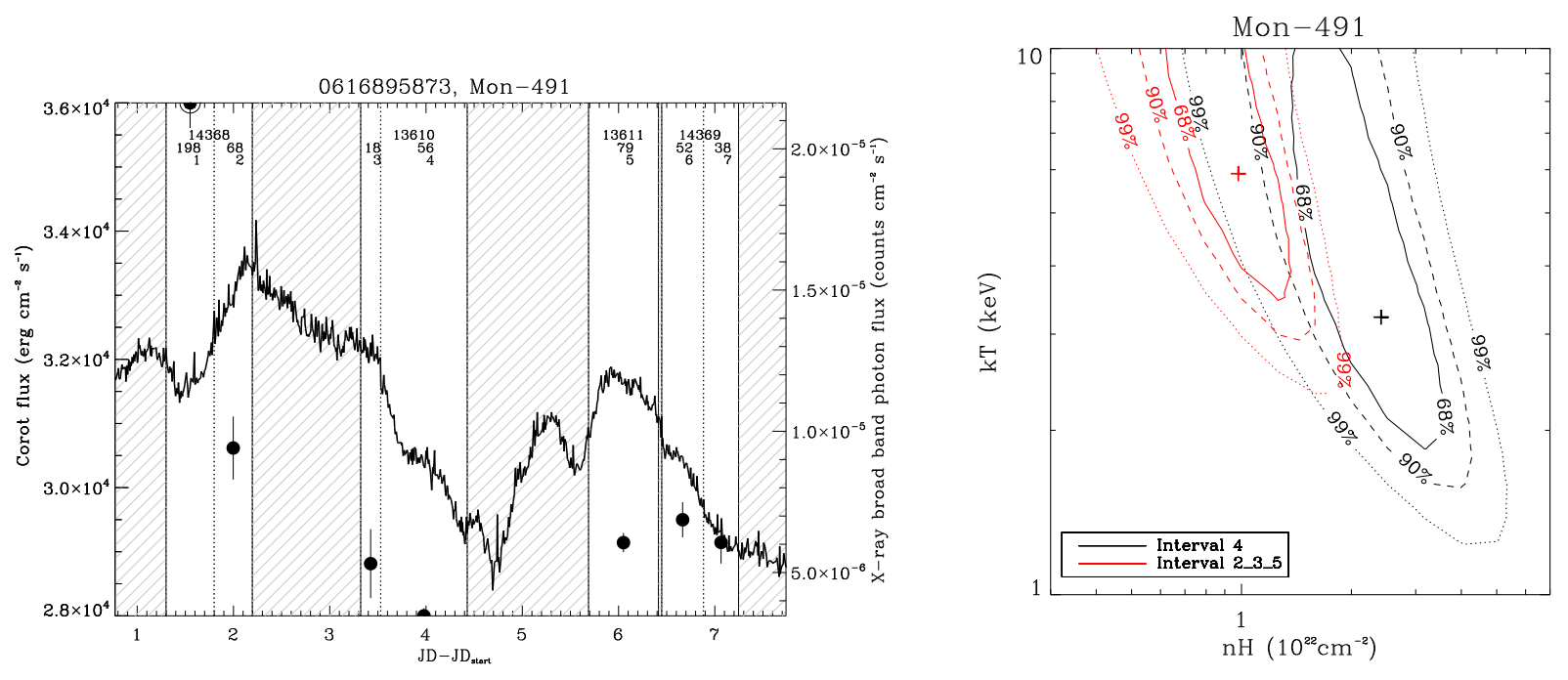

Fig. 10. Optical and X-ray variability of Mon-491, with panel format and content generally following Fig. 4. The contours are from the X-ray spectral fit of the spectrum observed during the interval \#4 (black) and \#2+\#3+\#5 (red).

- No evident features are observed in \#3 and \#4, even if there are several spikes that may be the consequence of unsteady accretion.

- A steep decline of the optical emission during \#5.

In the dip observed in the second Chandra frame the optical flux decreases by $10.4 \%$, corresponding to the extinction increasing by $0.14^{m}$. We observe the largest values of $E_{10 \%}$ and $E_{25 \%}$ during the large dip in the second Chandra frame, suggesting a larger X-ray absorption during the optical dip. Figure 9 also shows that the time variability of both the CoRoT and X-ray flux is coherent and correlated, as expected in AA Tau-like stars. The X-ray spectrum of Mon-619 is one of the hardest observed in our sample (Fig. A.5), with a median photon energy of $3.26 \mathrm{keV}$, (the typical value in our sample is $1.3 \mathrm{keV}$ ). Given the low X-ray counts, the spectral fits are not well constrained and thus not discussed.

Mon-491: Mon-491 is a K3V star actively accreting from its disk, and it has been classified by Cody et al. (2014) as a longterm variable and by Stauffer et al. (2016) as a star with variable accretion. The CoRoT light curve of Mon-491 (Fig. 10) shows:

- A rising phase in optical during \#1 and \#2.

- A very steep optical decline with the CoRoT flux decreasing by approximately $20 \%$ during the second Chandra frame (\#4), after a phase with a more constant optical flux (\#3), which may be due to variable extinction.

- An almost constant optical emission during \#5 and \#6 and then declining during \#7.

Even if the light curve of Mon-491 does not show dips during the Chandra frames, we want to verify whether or not during the decline of optical emission observed in \#4, there is evidence for increasing X-ray absorption. We compare the X-ray properties of Mon-491 during the intervals where the optical light curve does not decline (i.e., \#2+\#3+\#5) with those observed during $\# 4$. $N_{\mathrm{H}}$ is smaller during the former time intervals than during $\# 4$ at $\sim 96 \%$ confidence: $N_{\mathrm{H} \# 4}=2.14_{-0.45}^{+1.07} \times 10^{22} \mathrm{~cm}^{-2}$ while

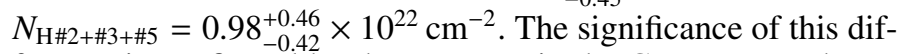
ference is confirmed by the contours in the C-stat space shown in Fig. 10.

Mon-774: the CoRoT light curve of Mon-774 (spectral type $\mathrm{K} 2.5$, classified as "stochastic" by Cody et al. 2014 with an
AA Tau phase observed during 2008 and analyzed in detail by McGinnis et al. 2015), shown in Fig. 11, is characterized by:

- A sequence of optical high and low phases in \#1, \#2, and \#3

- Two large optical dips, one dominating the third Chandra frame (time intervals \#7 and \#8), and one between the first and second Chandra frames.

- An optical dip observed during \#10 after a higher phase in \#9.

- A small optical dip observed in \#5 during the decline phase from \#4 to \#6.

The CoRoT flux variation during the dip in \#7 is approximately $16.8 \%$, corresponding to an increase of optical extinction $\Delta A_{V}=0.2^{m}$, while the dip observed between the first and second Chandra frames is less deep, with a $6.4 \%$ CoRoT flux variation $\left(\Delta A_{V}=0.09^{m}\right)$. The variability of the $\mathrm{X}$-ray properties is shown in the right panels of Fig. 11. $N_{\mathrm{H}}$ is larger than zero in three time intervals. In the interval \#10, the best fit predicts a $N_{\mathrm{H}}$ larger than zero but the C-stat contours, not shown here, admit solutions with $\mathrm{N}_{H}=0$ within a $68 \%$ confidence level; this is, therefore, not discussed further here.

In the interval \#8, $N_{\mathrm{H}}$ is only slightly larger than zero at $68 \%$ confidence. Interval \#8 is actually part of the large dip that dominates \#7, so the two intervals must be considered together. However, the X-ray spectral fit of the spectrum observed during $\# 7+\# 8$ does not result in a well-constrained estimate of $N_{\mathrm{H}}$, with solutions ranging from 0 to approximately $0.3 \times 10^{22} \mathrm{~cm}^{-2}$ within $68 \%$ confidence.

The small dip isolated in the time interval \#5 is more interesting in this respect. The $N_{\mathrm{H}}$ obtained using $2 \mathrm{~T}$ thermal plasma model (using a 1T model, the fit is not statistically acceptable) $\left(1.07_{-0.19}^{+0.19} \times 10^{22} \mathrm{~cm}^{-2}\right)$ is significantly different from zero and larger than the value obtained from the average spectrum $\left(0.54_{-0.24}^{+0.25} \times 10^{22} \mathrm{~cm}^{-2}\right)$ at a $68 \%$ confidence level, as proved by the $\mathrm{C}$-stat contours shown in Fig. 11 .

Mon-1076: the CoRoT light curve of the non-accreting star Mon-1076 (spectral type M1), listed as a star with periodic flux dips in Stauffer et al. (2015), is shown in Fig. 12 and is characterized thus:

- During the second Chandra frame, the CoRoT light curve is dominated by a large dip (time intervals \#2, \#3, and \#4), with 

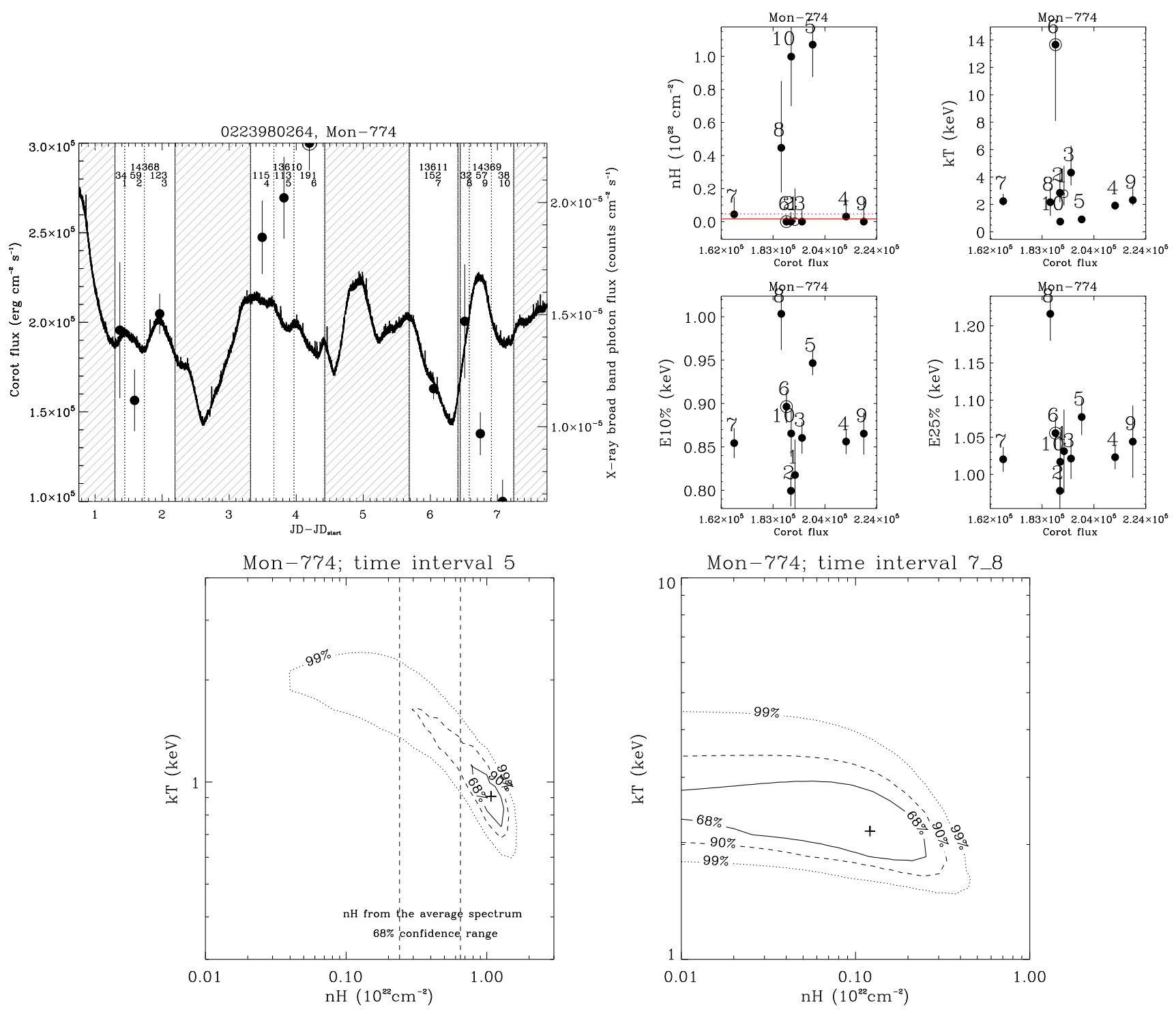

Fig. 11. Optical and X-ray variability of Mon-774, with panel format and content generally following Fig. 4. The contours are from the X-ray spectral fit of the spectrum observed during the interval \#5 (left bottom panel) and \#7+\#8 (right bottom panel). The vertical dashed lines in the top panel mark the $68 \%$ confidence range for the $N_{\mathrm{H}}$ obtained fitting the average spectrum.

a $10.6 \%$ CoRoT flux variation (corresponding to an increase of optical extinction by $\Delta A_{V}=0.15^{\mathrm{m}}$, see Table 6).

- An optical and X-ray flare is isolated in the interval \#3.

- Almost constant optical emission is observed during \#1, \#5, and \#6 (in the latter case probably with some variability).

The variability of the X-ray properties, shown in the right panels in Fig. 12, suggests a larger $N_{\mathrm{H}}$ observed at the beginning of the optical dip, compared with the remaining intervals. The significance of this difference is confirmed in the bottom panel of Fig. 12, which compares the confidence regions in the C-stat space obtained from the fit of the X-ray spectrum observed in the intervals \#2+\#4 (during the dip) and \#5+\#6 (after the dip). The X-ray absorption in the former interval is larger than that observed in the latter within a $68 \%$ confidence.

\subsection{Variability of the X-ray properties during the optical bursts}

In this section, we analyze the time-resolved X-ray properties during the optical bursts. We look for evidence of: i) a soft component in the X-ray spectrum (below $1 \mathrm{keV}$ ) that may result from the emission of accreting gas in the accretion shock; and ii) increasing X-ray absorption due to the gas in the accretion streams. Hereafter, we classify the X-ray spectra whose observed $\mathrm{X}$-ray emission below $1 \mathrm{keV}$ is significantly larger than the flux predicted by the best fit 1T thermal plasma model fitting the observed time-resolved X-ray spectra as explained in Sect. 5.1 as having "an intense soft X-ray spectral component". With few exceptions, we restrict this definition to cases where the spectral fit using the $1 \mathrm{~T}$ thermal plasma model is statistically unacceptable, accounting for the fact that stellar coronae are not isothermal and $2 \mathrm{~T}$ models may always be required when there are enough X-ray photons detected.

To analyze the simultaneous optical and X-ray variability of stars with optical bursts, we used an approach similar to that adopted for the stars with dips. For each star, we show a set of panels including the CoRoT light curve observed during the Chandra frames, superimposed with the variability of the soft X-ray flux, the observed time-resolved X-ray spectra, and the variability of the following $\mathrm{X}$-ray properties: the plasma 

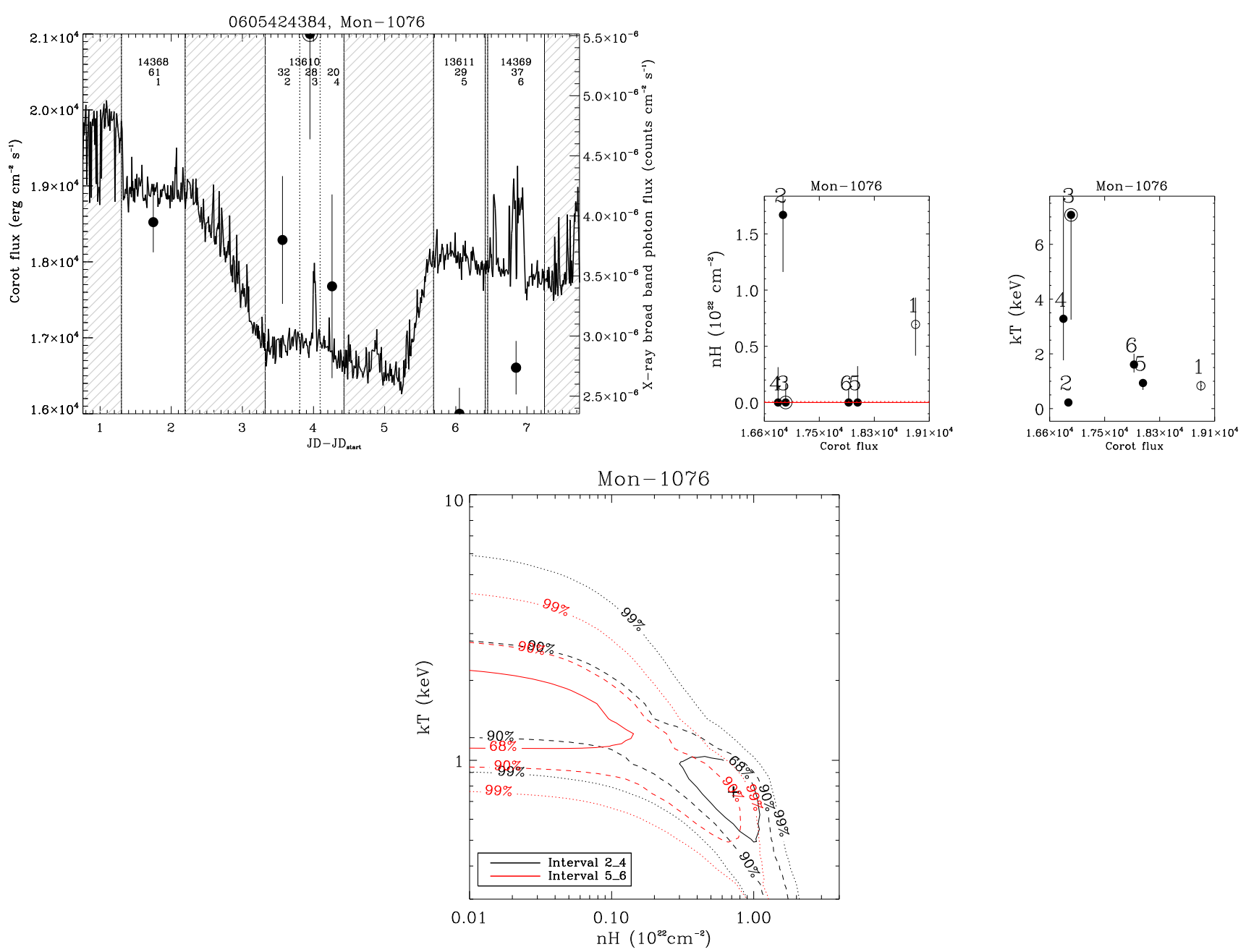

Fig. 12. Optical and X-ray variability of Mon-1076, with panel format and content generally following Fig. 4. The contours are from the X-ray spectral fit of the spectrum observed during the interval \#2+\#4 (black contours) and \#5+\#6 (red contours).

temperature, the $E_{10 \%}$ and $E_{25 \%}$ photon energy quantiles, and the median photon energy $E_{50 \%}$ for stars with soft X-ray emission during the optical bursts, or the hydrogen column density $N_{\mathrm{H}}$ for stars with increasing X-ray absorption during the optical bursts. Together with the time-resolved X-ray spectra, we show the best fit thermal plasma model, $1 \mathrm{~T}$, or $2 \mathrm{~T}$ if the fit using $1 \mathrm{~T}$ thermal models is statistically unacceptable.

The criterion adopted to search for a soft component (i.e., the acceptance level for 1T models) depends on plasma properties and on the number of photons collected. Since we aim to monitor the presence of a soft component irrespective of the photon statistics, for each star, we also analyzed the variability of the normalization of the soft component over the defined time intervals. To attempt highlighting only the variation of the normalization of the soft component and thus the variability of the cold plasma emission measure, for this test we fitted the timeresolved X-ray spectra with $2 \mathrm{~T}$ thermal plasma models with fixed $N_{\mathrm{H}}$ (set equal to the value derived from the known optical extinction), $k T_{\text {soft }}=0.3 \mathrm{keV}$ (typical of the soft emission from accretion spots), $k T_{\text {hard }}=1.6 \mathrm{keV}$ (the typical coronal temperature of NGC 2264 members). We do not set the plasma temperatures and/or the absorption in our search of intense soft X-ray spectral components during the optical bursts to avoid underestimating of the X-ray absorption that would affect our results.

Mon-808: Mon-808 is an accreting disk bearing star with spectral type K4 (Dahm \& Simon 2005) classified as a "burster" by Cody et al. (2014) and as a "burst-dominated light curve" by Stauffer et al. (2014). Its CoRoT light curve during the Chandra frames (Fig. 13) shows:

- Large optical bursts in the time intervals \#1 and \#2.

- Small optical bursts in the time intervals \#3 and \#8.

- A bright optical and X-ray flare dominating the time intervals \#4 and \#5 (the latter dominated by the decaying phase of the $\mathrm{X}$-ray flare).

- Possible micro bursts in \#6 and \#7, with a decline of the optical emission by approximately $3 \%$ observed in the latter.

In some of the time-resolved X-ray spectra shown in Fig. 13, the soft $\mathrm{X}$-ray emission below $0.8 \mathrm{keV}$ is larger than the prediction of the 1 T fitting model, specifically in \#1, \#2, and \#3. The bottom panel shows how the normalization of the soft component of the best fit $2 \mathrm{~T}$ thermal plasma model (with fixed $N_{\mathrm{H}}$ and $k T$ ) varies in the time intervals. The associated errors are too large to allow us any meaningful comparison.

Table 3 shows the results of the time-resolved spectral analysis, specifically the predicted plasma temperatures, and the nullhypothesis probabilities resulting from the best-fit $1 \mathrm{~T}$ and $2 \mathrm{~T}$ APEC models. In particular, in the time intervals \#1 and \#2, the use of 1T plasma models results in unacceptable fits, while the quality of the fit improves significantly using $2 \mathrm{~T}$ plasma models. In the time intervals \#3 and \#8, the $1 \mathrm{~T}$ fit is acceptable (likely 

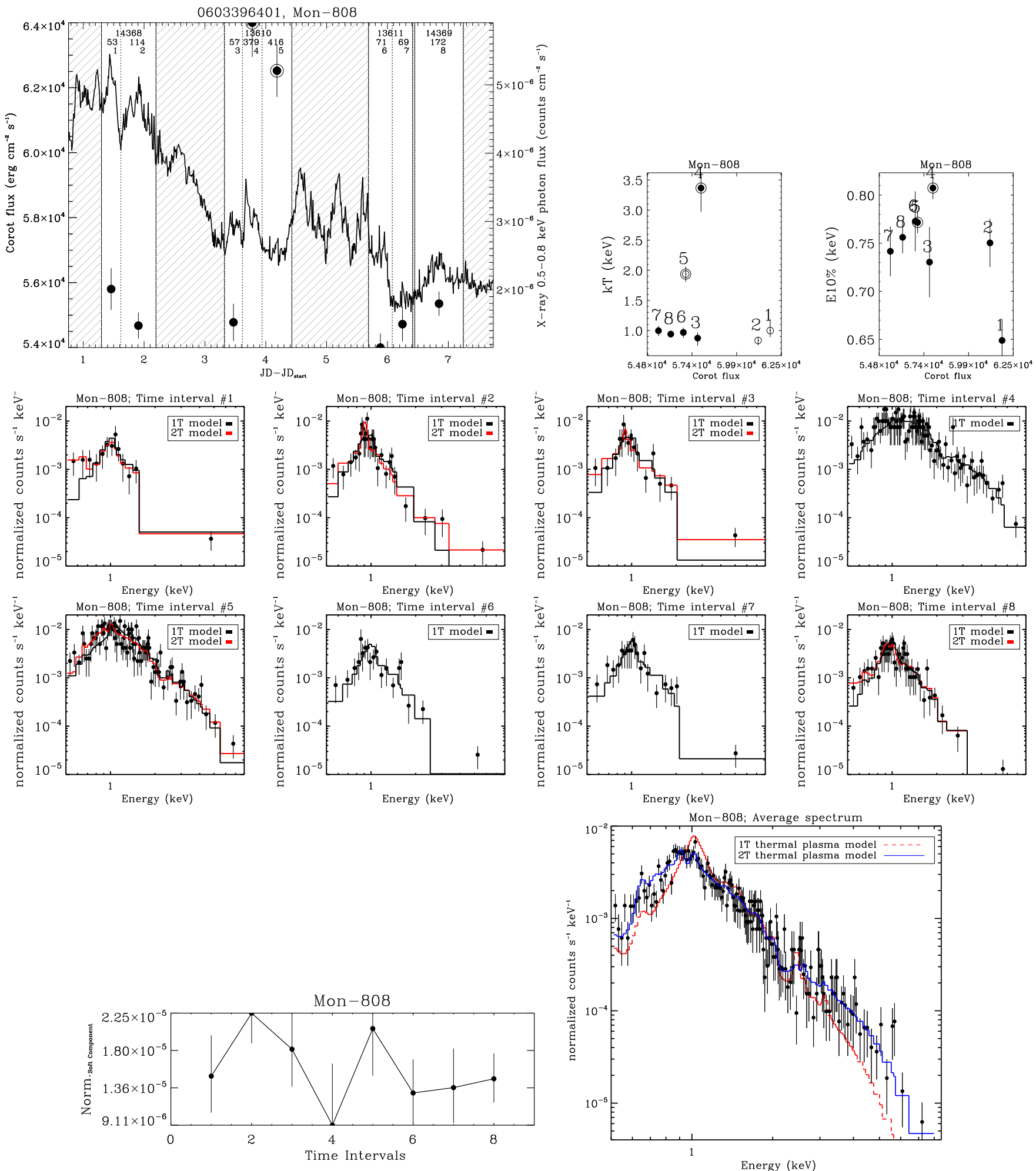

Fig. 13. Optical and X-ray variability of Mon-808, with panel format and content generally following Fig. 4. There are two differences with respect to prior figures: i) the upper right panels show the variability of $k T$ and $E_{10 \%}$ in units of $\mathrm{keV}$; and ii) the dots in the upper left panel show the $\mathrm{X}$-ray photon flux in the $0.5-0.8 \mathrm{keV}$ energy band. The central panels show the X-ray spectra observed in the time intervals (dots) with the best fit 1T thermal plasma model (black line), together with the $2 \mathrm{~T}$ model (red line) when needed. The bottom left panel shows the normalization of the soft component obtained by fitting the X-ray spectrum observed in the time intervals with the $2 \mathrm{~T}$ thermal plasma model, with plasma temperatures fixed at $k T_{\text {soft }}=0.3 \mathrm{keV}$ and $k T_{\text {hard }}=1.6 \mathrm{keV}$ and $N_{\mathrm{H}}$ fixed at the value obtained from the optical extinction $\left(0.009 \times 10^{22} \mathrm{~cm}^{-2}\right)$. The bottom right panel shows the average X-ray spectrum (dots). The dashed red line shows the best fit 1T thermal plasma model, while the blue line is the 2T model.

due in the former to the low X-ray counts), but the fitting thermal plasma model does not reproduce the observed soft X-ray emission (below $0.7 \mathrm{keV}$ in the former and below $0.9 \mathrm{keV}$ in the latter), which are instead well-fitted with the $2 \mathrm{~T}$ thermal plasma 
Table 3. Predicted plasma temperatures for Mon-808 from the timeresolved X-ray spectral fits.

\begin{tabular}{cccrc}
\hline \hline $\begin{array}{c}\text { Interval } \\
\#\end{array}$ & $k \mathrm{~T} 1$ & $k \mathrm{~T} 2$ & $P(1 \mathrm{~T})$ & $P(2 \mathrm{~T})$ \\
$\mathrm{keV}$ & $\mathrm{keV}$ & \multicolumn{1}{c}{$\%$} & $\%$ \\
\hline 1 & $0.16_{-0.12}^{+0.13}$ & $1.2_{-0.03}^{+0.4}$ & 2.1 & 98.8 \\
2 & $0.12_{-0.03}^{+0.08}$ & $4.6_{-3.50}$ & 0.3 & 50.0 \\
3 & $0.87_{-0.07}^{+0.10}$ & & 23.3 & \\
4 & $3.37_{-0.40}^{+0.54}$ & & 54.8 & \\
5 & $0.42_{-0.11}^{+0.11}$ & $2.6_{-0.51}^{+1.39}$ & 0.7 & 26.8 \\
6 & $0.97_{-0.09}^{+0.07}$ & & 22.2 & \\
7 & $1.00_{-0.09}^{+0.08}$ & & 81.1 & \\
8 & $0.94_{-0.16}^{+0.06}$ & & 55.4 & \\
\hline
\end{tabular}

Notes. The results from the $2 \mathrm{~T}$ model are shown when the best fit with the $1 \mathrm{~T}$ model is poorly constrained.

model. These are the time intervals where we search for soft $\mathrm{X}$-ray emission due to accretion.

Since we want to constrain the soft temperature as well as possible, we fit the average X-ray spectrum over the time intervals where an intense soft X-ray spectral component is observed using the $2 \mathrm{~T}$ thermal plasma model. An acceptable fit is obtained from the average spectrum observed in \#1+\#2+\#8, with a soft temperature $k T_{1}=0.15_{-0.04}^{+0.06} \mathrm{keV}\left(P_{\%}=91 \%\right)$, which corresponds to $1.7_{-0.04}^{+0.07} \mathrm{MK}$. We obtain the same soft component temperature $\left(k T_{1}=0.14_{-0.05}^{+0.04} \mathrm{keV}\right)$ from the average \#1+\#2 $\mathrm{X}$-ray spectrum, but with a less constrained X-ray spectral fit $\left(P_{\%}=8 \%\right)$. In the approximation of strong shock scenario, the pre-shock velocity can be calculated from the post-shock plasma temperature as:

$v_{\text {pre }}^{2}=\frac{16 k T_{\text {post }}}{3 \mu m_{\mathrm{H}}}$

where $v_{\text {pre }}$ is the pre-shock gas velocity, $T_{\text {post }}$ the post-shock temperature, and $\mu$ the mean molecular weight ( 0.61 in our case). Adopting the computed soft temperature as $T_{\text {post }}$, this calculation results in a pre-shock velocity of the accreting gas of $352_{-48}^{+68} \mathrm{~km} \mathrm{~s}^{-1}$. Adopting a stellar mass of $1.2 M_{\odot}$ and radius of $1.7 R_{\odot}$ obtained by interpolating the Siess et al. (2000) PMS isochrones with the values of $L_{\mathrm{bol}}$ and $T_{\mathrm{eff}}$ for Mon-808 (the former from a bolometric correction on the dereddened $I$ magnitude and the latter from the known spectral type), an age of 4.5 Myr, and the hypothesis of negligible energy loss during the accretion, this pre-shock velocity corresponds to a free-fall from a distance of $3.2_{-0.6}^{+1.7} R_{\odot}$, corresponding to $1.9_{-0.4}^{+1.0}$ stellar radii, calculated from the center of the system. The fact that the pre-shock velocity we obtain is significantly smaller than the free-fall velocity from infinity $\left(519 \mathrm{~km} \mathrm{~s}^{-1}\right)$ suggests that the free-fall radius $\left(R_{\mathrm{ff}}\right)$ is sufficiently well constrained. Its lower limit is comparable with the disk inner radius (1.4 stellar radii) which can be predicted by SED analysis (see Sect. 5.1). Since the light curve of Mon-808 is not periodic, its rotation period is unknown, and thus we cannot calculate the co-rotation radius as $\left(G M_{\text {star }}\right)^{1 / 3} \times\left(P_{\text {star }} / 2 \pi\right)^{2 / 3}$. However, bearing in mind that this calculation is strongly uncertain, we note that the free-fall radius is smaller than the typical co-rotation radii of disk-bearing stars (5-10 $R_{\text {star }}$, Hartmann et al. 1998; Shu et al. 2000). The presence of a significant soft X-ray spectral component below $0.9 \mathrm{keV}$ in Mon-808 is also evident in the average spectrum, shown in
Fig. 13. Together with the observed spectrum (with flares removed), we show the best fit $1 \mathrm{~T}$ thermal plasma model, which results in a poor fit $\left(P_{\%} \sim 0\right)$, and the best fit $2 \mathrm{~T}$ model, which results in an acceptable fit $\left(P_{\%}=55 \%\right)$ with the two plasma temperatures $k T_{1}=0.37_{-0.04}^{+0.03} \mathrm{keV}$ and $k T_{2}=2.6_{-0.2}^{+0.3} \mathrm{keV}$.

Mon-370: the K5 star Mon-370 is among the strongest accretors in NGC 2264, with a $\mathrm{H} \alpha \mathrm{EW}$ approximately corresponding to the $71 \%$ quantile of the $\mathrm{H} \alpha \mathrm{EW}$ distribution of all the accretors in our sample. The CoRoT light curve of this star (Fig. 14) shows:

- A superposition of intense optical bursts in \#4.

- That in \#5 we isolate the beginning of the rising phase of a burst occurring between the second and third Chandra frame.

- An X-ray and optical flare during \#6.

- A decline of optical emission by $9.1 \%$ between \#9 and \#10.

- Myriad small peaks during all the other intervals.

We can speculate that the light curve of this star is affected by myriad small bursts caused by an intense and chaotic accretion process, with several short-lived accretion streams hitting the stellar surface at various positions. The X-ray spectra observed in most of the time intervals (shown in Fig. 14) confirm this scenario, showing an intense soft X-ray spectral component (typically for energies $\leq 0.8 \mathrm{keV}$, sometimes $\leq 1 \mathrm{keV}$ ). The most evident soft X-ray spectral component is observed in the time interval \#4, characterized by the most intense optical burst and the lowest $E_{10 \%}$ observed in this star. A smaller but evident soft Xray spectral component is also present in the time intervals \#7 and \#10, all characterized by several small bursts. The X-ray spectral fit with $1 \mathrm{~T}$ thermal plasma model is not well-constrained in most of the cases (fourth column in Table 4): in the time intervals \#1, \#2, \#4, \#7 and \#10 $P_{\%}$ is below the 5\% threshold. In some cases, this is likely due to the fact that we have enough $\mathrm{X}$-ray photons to resolve different thermal components in the coronal emission (e.g., \#2); in other cases by the presence of an evident soft X-ray spectral component (e.g. \#4). An intense soft $\mathrm{X}$-ray spectral component is also evident in the average spectrum, as shown in the bottom right panel of Fig. 14.

The bottom left panel in Fig. 14 shows the time variability of the normalization of the soft component using the $2 \mathrm{~T}$ model with fixed $N_{\mathrm{H}}$ and kT. The time interval with the largest normalization of the soft component is \#5, where we isolate the rising part of a burst, followed by \#6 dominated by a X-ray flare. The normalization observed in \#4 and \#7 is instead compatible with those observed in the remaining intervals.

As for Mon-808, we fit the X-ray spectra of these time intervals with two temperatures plasma models, which better reproduce the observed spectra, primarily in the time intervals \#4 and \#7, but also in \#2 and \#10 (see Fig. 14 and Table 4). The temperatures predicted by the best-fit models are shown in Table 4, together with the pre-shock velocity calculated using Eq. (2) and the corresponding free-fall launching distance from the central star, in units of stellar radii. In the time intervals \#2, \#4, $\# 7$, and \#10, the pre-shock velocity ranges from $242 \mathrm{~km} \mathrm{~s}^{-1}$ to $557 \mathrm{~km} \mathrm{~s}^{-1}$, sometimes being compatible with the free-fall velocity from infinity $\left(512 \mathrm{~km} \mathrm{~s}^{-1}\right)$. In order to better constrain these parameters, we fit the average X-ray spectrum observed summing these four time intervals, obtaining a good fit with a $2 \mathrm{~T}$ thermal plasma model $\left(P_{\%} \sim 100 \%\right)$ with $k T_{1}=0.16_{-0.04}^{+0.08} \mathrm{keV}$ and $k T_{2}=1.86_{-0.29}^{+0.55} \mathrm{keV}$. The soft temperature corresponds to a pre-shock velocity of $366_{-49}^{+83} \mathrm{~km} \mathrm{~s}^{-1}$ from a free-fall radius of $2.0_{-0.4}^{+2.3} R_{\text {star }}$. The X-ray spectral fit of the average spectrum 
M. G. Guarcello et al.: CSI 2264: Simultaneous optical and X-ray variability in pre-main sequence stars
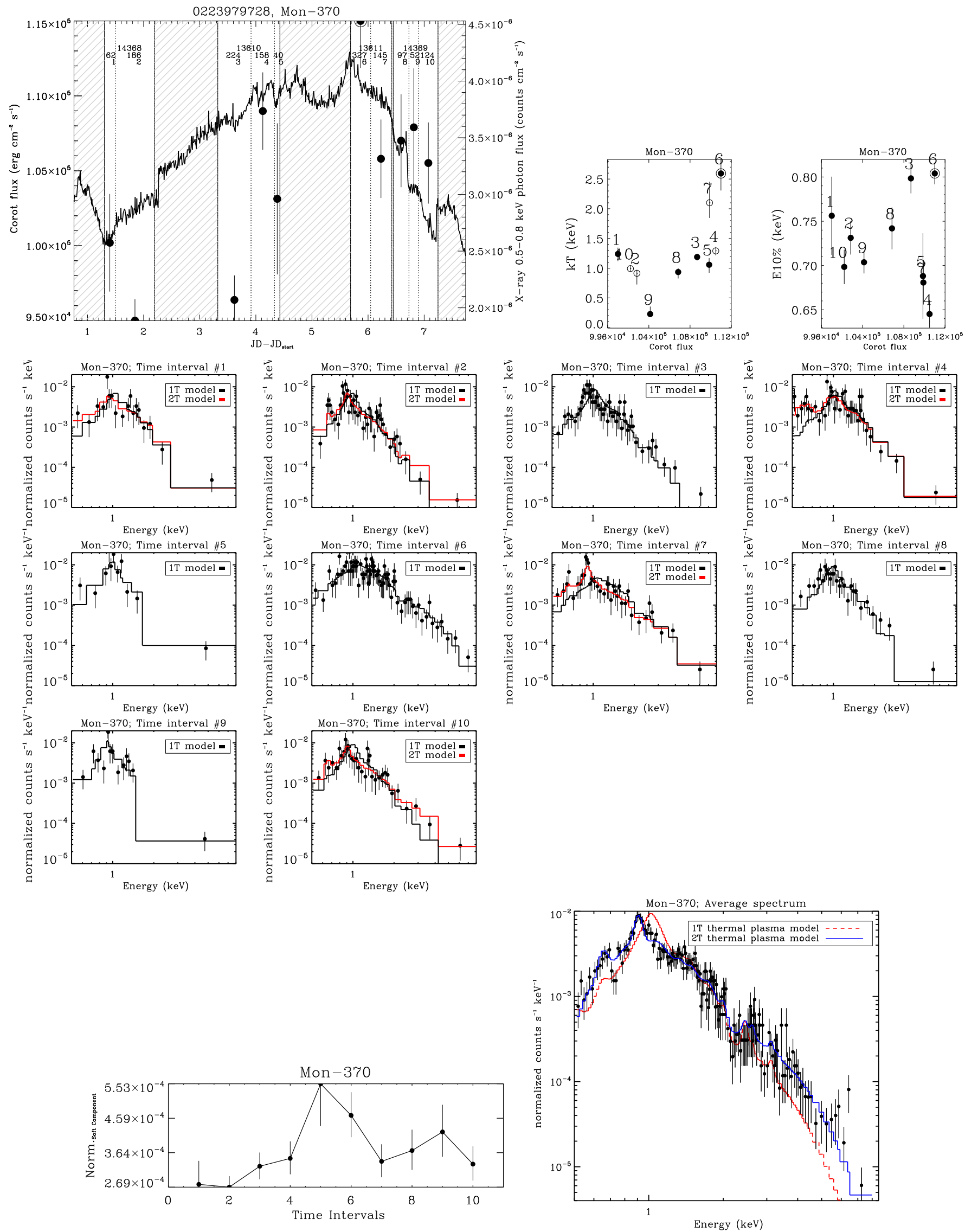

Fig. 14. Time variability of the optical flux and X-ray properties of Mon-370 with the panel format and content as in Fig. 13. 

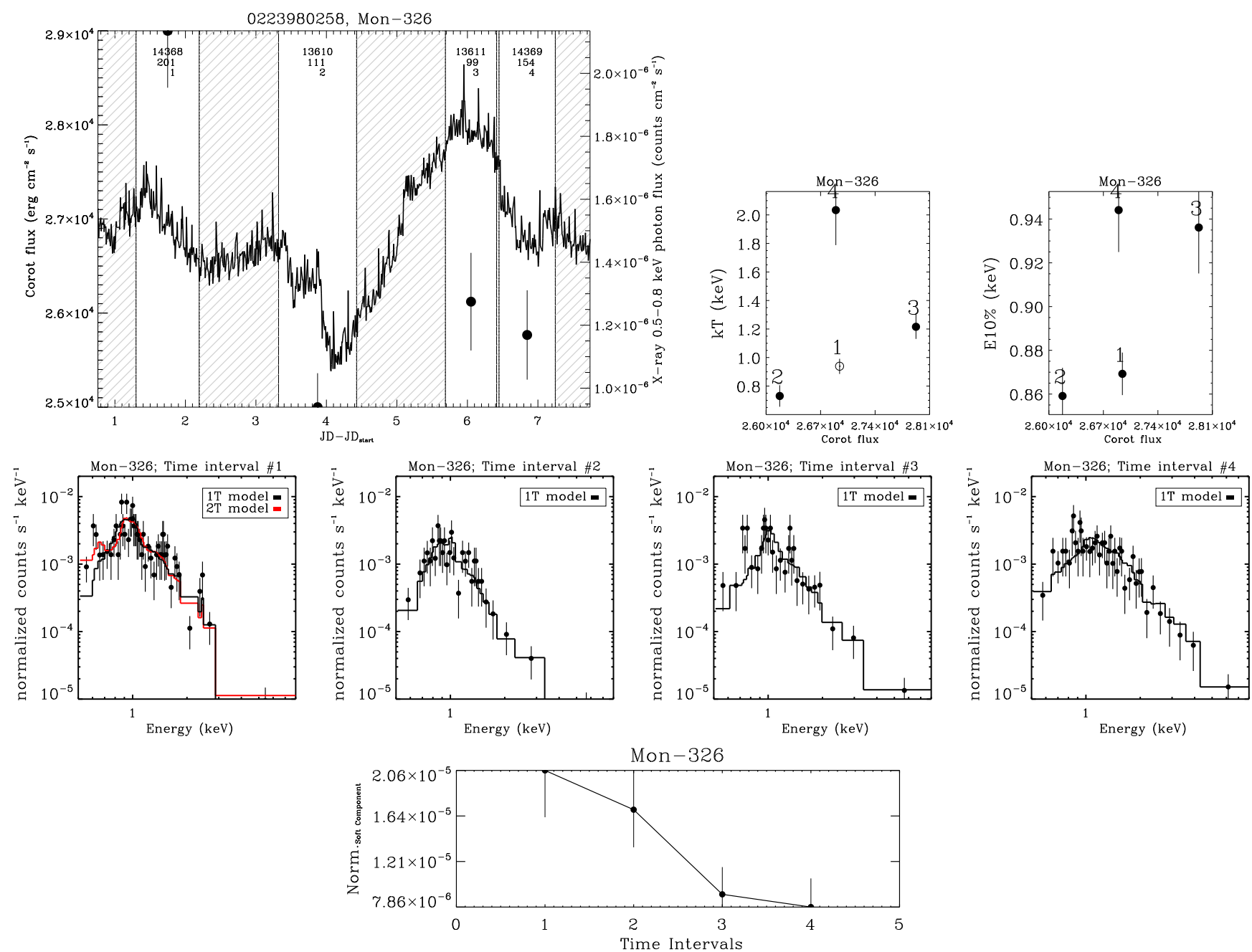

Fig. 15. Time variability of the optical flux and X-ray properties of Mon-326 with the panel format and content as in Fig. 13.

Table 4. Predicted plasma temperatures for Mon-370 from the timeresolved X-ray spectral fits.

\begin{tabular}{rccrccc}
\hline \hline$\#$ & $k \mathrm{~T} 1$ & $k \mathrm{~T} 2$ & $P(1 \mathrm{~T})$ & $P(2 \mathrm{~T})$ & $v_{\text {pre }}$ & $R_{\mathrm{ff}}^{*}$ \\
& $\mathrm{keV}$ & $\mathrm{keV}$ & \multicolumn{1}{c}{$\%$} & \multicolumn{1}{c}{$\%$} & $\mathrm{~km} \mathrm{~s}^{-1}$ & \multicolumn{1}{c}{$R_{\text {star }}$} \\
\hline 1 & $0.07_{-0.05}^{+0.17}$ & $0.90_{-0.22}^{+1.65}$ & 4.6 & 48.4 & $242_{-113}^{+207}$ & $4.5_{-3.2}^{+11.2}$ \\
2 & $0.22_{-0.11}^{+0.11}$ & $1.93_{-0.60}^{+1.81}$ & 0.0 & 7.8 & $429_{-187}^{+97}$ & $1.4_{-0.5}^{+3.1}$ \\
3 & $1.19_{-0.06}^{+0.05}$ & & 8.7 & & & \\
4 & $0.15_{-0.06}^{+0.06}$ & $1.42_{-0.44}^{+0.45}$ & 0.0 & 25.4 & $355_{-80}^{+65}$ & $2.1_{-0.6}^{+1.4}$ \\
5 & $1.06_{-0.11}^{+0.11}$ & & 12.7 & & & \\
6 & $2.59_{-0.28}^{+0.41}$ & & 7.8 & & & \\
7 & $0.14_{-0.06}^{+0.08}$ & $2.45_{-0.89}^{+2.29}$ & 0.0 & 92.4 & $342_{-83}^{+87}$ & $2.2_{-0.8}^{+1.7}$ \\
8 & $0.94_{-0.11}^{+0.06}$ & & 76.8 & & & \\
9 & $0.23_{-0.05}^{+0.22}$ & & 16.2 & & & \\
10 & $0.20_{-0.11}^{+0.17}$ & $2.61_{-1.08}^{+3.74}$ & 0.13 & 97.8 & $409_{-134}^{+148}$ & $1.6_{-0.8}^{+1.9}$ \\
\hline
\end{tabular}

Notes. The results from the $2 \mathrm{~T}$ model are shown when the best fit with the $1 \mathrm{~T}$ model is poorly constrained. ${ }^{*} R_{\mathrm{ff}}$ is calculated assuming $M_{\text {star }}=$ $1.13 M_{\odot}, R_{\mathrm{star}}=1.64 R_{\odot}$, and age $=4 \mathrm{Myr}$.

observed in the entire Chandra observation results in similar temperatures $\left(k T_{1}=0.19_{-0.03}^{+0.02} \mathrm{keV}\right.$ and $\left.k T_{2}=1.95_{-0.18}^{+0.18} \mathrm{keV}\right)$. Bearing in mind that this calculation is approximate, the free-fall velocity is smaller than the value from infinity, the upper limit of $R_{\mathrm{ff}}$ is well below both the inner radius of the dusty disk predicted by the SED analysis (8.9 stellar radii), and the co-rotation radius calculated adopting the rotation period of 11.84 days (Venuti et al., in prep.), is equal to $13.8 R_{\text {star }}$.

Mon-119: marginal evidence for an intense soft X-ray spectral component during the optical bursts is also observed in Mon119 (see Fig. 8). This star has been discussed in Sect. 5.3. We focus here on the optical bursts during the time intervals \#3, \#7, and \#9. In particular, as shown in the right panels of Fig. 8, during \#3 and \#9, both the plasma temperature and the $10 \%$ and $25 \%$ energy quantiles suggest that the corresponding X-ray spectra are dominated by soft photons (see the spectra shown in Fig. A.4 and also in the variability of $E_{10 \%}$ shown in Appendix B). However, given the few X-ray photons detected, the two spectra are well fitted by 1T thermal plasma models $\left(P_{\%}=84 \%\right.$ and $93 \%$, respectively), as well as the average spectrum summing these two time intervals $\left(P_{\%}=58 \%\right)$.

Mon-326: the M0 star Mon-326, whose variability is shown in Fig. 15, is described as a star whose light curve is dominated by accretion hot spots in Stauffer et al. (2014). It is not a strong accretor and in fact its CoRoT light curve does not show many prominent bursts, except a 0.3 day-long burst observed at the beginning of the first Chandra frame (time interval \#1). In the first time interval, an intense soft X-ray spectral component is 

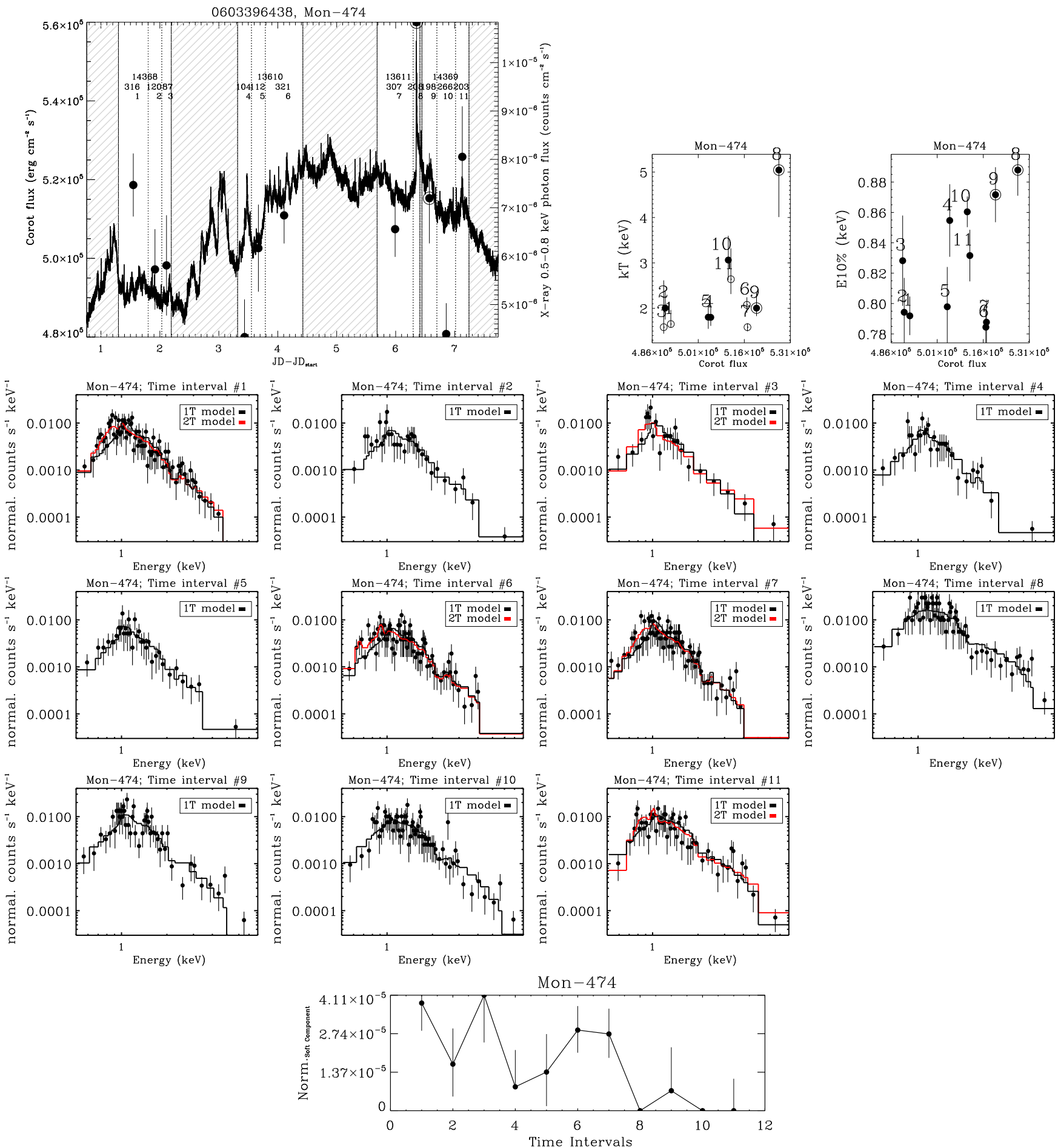

Fig. 16. Time variability of the optical flux and X-ray properties of Mon-474 with the panel format and content as in Fig. 13.

evident (for $k T \leq 0.7 \mathrm{keV}$ ), and the normalization of the soft component of the best fit $2 \mathrm{~T}$ model is larger than in the other intervals (bottom panel).

The fit with a $2 \mathrm{~T}$ plasma model of the X-ray spectrum observed in the first time interval (shown in Fig. 15) better reproduces the observed soft X-ray emission below $0.7 \mathrm{keV}$, with the null-hypothesis probability increasing from almost zero to $34 \%$. In \#1, the soft temperature obtained from the best fit $2 \mathrm{~T}$ model is $k T_{\text {soft }}=0.15_{-0.08}^{+0.10} \mathrm{keV}$. Using Eq. (2), this temperature corresponds to a pre-shock velocity of $355_{-113}^{+102} \mathrm{~km} \mathrm{~s}^{-1}$, compatible with the free-fall velocity from infinity $\left(395 \mathrm{~km} \mathrm{~s}^{-1}\right)$.
Mon-474: Fig. 16 shows the variability of Mon-474, the only G-type star (Dahm \& Simon 2005) analyzed in this section, which is actively accreting from its disk. This star was listed as a star with accretion bursts in Stauffer et al. (2014). The CoRoT light curve during the Chandra observations (but also in the remainder) is dominated by several small bursts:

- An intense optical burst dominates \#4 with the optical emission increasing by $3.8 \%$.

- In \#5, we isolate a transition from the large optical burst observed in \#4 and a sequence of small bursts. 
Table 5. Predicted plasma temperatures for Mon-474 from the timeresolved X-ray spectral fits.

\begin{tabular}{rccrc}
\hline \hline Interval & $\mathrm{T} 1$ & $\mathrm{~T} 2$ & $P(1 \mathrm{~T})$ & $P(2 \mathrm{~T})$ \\
\hline & $\mathrm{keV}$ & $\mathrm{keV}$ & \multicolumn{1}{c}{$\%$} & $\%$ \\
\hline 1 & $0.66_{-0.35}^{+0.16}$ & $2.65_{-0.65}^{+1.54}$ & 1.9 & 88.0 \\
2 & $2.01_{-0.26}^{+0.27}$ & & 63.7 & \\
3 & $0.87_{-0.19}^{+0.24}$ & $23.1_{-19.98}$ & 1.7 & 79.4 \\
4 & $1.80_{-0.19}^{+0.28}$ & & 76.5 & \\
5 & $1.80_{-0.25}^{+0.26}$ & & 98.7 & \\
6 & $0.25_{-0.09}^{+0.10}$ & $2.16_{-0.45}^{+0.69}$ & 0.5 & 26.9 \\
7 & $0.78_{-0.38}^{+0.21}$ & $2.27_{-0.45}^{+1.15}$ & 3.5 & 64.2 \\
8 & $5.05_{-1.04}^{+1.68}$ & & 83.6 & \\
9 & $2.01_{-0.18}^{+0.25}$ & & 11.0 & \\
10 & $3.06_{-0.44}^{+0.54}$ & & 13.2 & \\
11 & $0.54_{-0.15}^{+0.09}$ & $6.73_{-3.76}$ & 0.0 & 8.4 \\
\hline
\end{tabular}

Notes. The results from the $2 \mathrm{~T}$ model are shown when the best fit with the $1 \mathrm{~T}$ model is poorly constrained.

- The intervals \#1, \#2, \#3, \#6, \#7, \#10,\#11 are dominated by several small optical bursts.

- A bright optical and X-ray flare dominate \#8 and \#9.

The time-resolved X-ray spectra (central panels of Fig. 16) show an intense soft X-ray spectral component below $1 \mathrm{keV}$ in the time intervals \#1 and \#6. This may also be present even if less evident during the interval \#7. No evident soft X-ray spectral component is observed during \#3 and \#11. The X-ray spectra observed in some of these intervals such as \#6 and \#7 are not well-fit with $1 \mathrm{~T}$ thermal plasma models, and they also show soft X-ray photon energy quantiles. Table 5 shows the plasma temperatures predicted by the best fit $1 \mathrm{~T}$ or $2 \mathrm{~T}$ (when the former is poorly constrained) plasma model and the associated null-hypothesis probabilities.

Repeating the calculation made for Mon-808 for Mon-474 and adopting a stellar mass of $1.9 M_{\odot}$ and a radius of $4.04 R_{\odot}$, we obtain a pre-shock velocity of the accreting material of $744_{-334}^{+85} \mathrm{~km} \mathrm{~s}^{-1}$ in time interval \#1 and $458_{-92}^{+84} \mathrm{~km} \mathrm{~s}^{-1}$ in time interval \#6, both compatible with the free-fall velocity from infinite distance from the star $\left(423 \mathrm{~km} \mathrm{~s}^{-1}\right)$.

Mon-357: the variability of the K5 star Mon-357 is shown in Fig. 17. Even though it has a small $\mathrm{H} \alpha \mathrm{EW}$, this star has a CoRoT light curve dominated by several burst-like features of various intensities:

- A bright optical and X-ray flare is observed during the time interval \#1.

- Large optical bursts are observed during \#5, \#6, \#7, and \#9.

- Small optical bursts are observed during \#2 and \#10.

- No interesting features are observed during \#3, \#4, and \#8.

The variability of the X-ray properties (Fig. 17) suggests that the $\mathrm{X}$-ray spectrum becomes softer and the X-ray emitting plasma colder with increasing CoRoT flux (ignoring the flare). The Spearman's rank correlation test is significant for $k T$ (correlation coefficient $\rho=-0.72$ and the two-side significance of its deviation from zero $P(\rho)=0.03), E_{25 \%}(\rho=-0.78, P(\rho)=0.01)$, and $E_{50 \%}(\rho=-0.70, P(\rho)=0.04)$, while it is not significant for $E_{10 \%}$, suggesting that the observed variability is dominated by accretion. Intense soft X-ray spectral components in the timeresolved X-ray spectra are observed in some intervals. In \#7, when the CoRoT light curve is dominated by a superposition of small bursts, it is necessary to adopt a $2 \mathrm{~T}$ thermal plasma model to obtain a good fit $\left(P_{\%}=3.3 \%\right.$ with the $1 \mathrm{~T}$ model, $93.2 \%$ with the $2 \mathrm{~T}$ model). No useful information is provided by the time variability of the normalization of the soft component of the best fit 2T model (not shown) because of the large associated errors.

The X-ray spectrum during \#7 shows a peak of soft X-ray emission at approximately $0.7 \mathrm{keV}$. The soft temperature predicted by the best fit $2 \mathrm{~T}$ thermal plasma model is well constrained, equal to $0.06_{-0.02}^{+0.01} \mathrm{keV}$. Since Chandra/ACIS-I is not sensitive to such soft emission, we do not calculate the preshock velocity from this plasma temperature. However, it must be noted that a similar value for the temperature of the soft component is also obtained from the average spectrum among those intervals with large bursts: \#5+\#7+\#9+\#10 ( $\left.P_{\%}=0.10\right)$, suggesting that the soft part of the X-ray spectrum of Mon-357 is dominated by emission from the accretion spots.

In Fig. 17 we show the average X-ray spectrum of Mon-357 with the best fit $1 \mathrm{~T}\left(P_{\%}=0 \%\right)$ and $2 \mathrm{~T}\left(P_{\%}=88 \%\right)$ thermal models. The presence of an intense soft X-ray spectral component is evident.

The search for increasing X-ray absorption during the optical bursts has been less prolific. This is, however, not surprising given that this effect can be observed only if the accretion streams obscure the coronal active regions when the accretion hot spots are clearly visible, which is strongly dependent on the geometry of the accretion and the distribution of the active regions in the stellar corona. In only two cases (Mon-945 and Mon771), described below, is there evidence for such a correlation.

Mon-945: Mon-945 is a K4 accreting star, with evidence for a larger veiling when the star is optically brighter, which supports the classification as "burster" by Cody et al. (2014) and Stauffer et al. (2014). The CoRoT light curve during the Chandra observations is dominated by a large number of bursts (Fig. 18):

- Optical bursts are observed in \#2, \#5, \#8, \#9, and \#10.

- \#1 and \#7 show dip-like optical features, more prominent in the former interval.

- \#3, \#4, and \#6 are dominated by optical and X-ray flares.

The bottom panels of Fig. 18 show the X-ray spectra observed during the time intervals, where there is no evidence of intense soft X-ray spectral components. However, of particular interest is the burst/flare observed during time interval \#3. The identification of this event as an X-ray flare by our automatic routines is uncertain given that the rising part is not observed by Chandra, as it lies between the first and second Chandra frames, and we observe only the decaying phase at the beginning of the second Chandra frame (the time interval \#3). Additionally, in \#3 we observe the highest value of X-ray absorption $\left(N_{\mathrm{H}}=0.91_{-0.36}^{+0.14} \times 10^{22} \mathrm{~cm}^{-2}\right)$ and a low plasma temperature $\left(k T=0.93_{-0.16}^{+0.09} \mathrm{keV}\right)$, similar to that observed in other intervals. These properties are more compatible with an accretion burst rather than an X-ray flare, with increasing X-ray absorption due to the accreting gas falling into the line of sight. This is the only time interval where the $N_{\mathrm{H}}$ obtained from spectral fit is significantly larger than zero, together with that observed in \#5 $\left(N_{\mathrm{H}}=0.47_{-0.14}^{+0.30} \times 10^{22} \mathrm{~cm}^{-2}\right)$ during which the CoRoT light curve shows several bursts. Figure. 18 also shows the contours in the C-stat space obtained for Mon-945 during the time intervals \#3 and \#5, supporting the evidence for larger $N_{\mathrm{H}}$.

Mon-771: the moderately accreting K4 star Mon-771 was not classified as a "burster" by Cody et al. (2014). However, its 
M. G. Guarcello et al.: CSI 2264: Simultaneous optical and X-ray variability in pre-main sequence stars
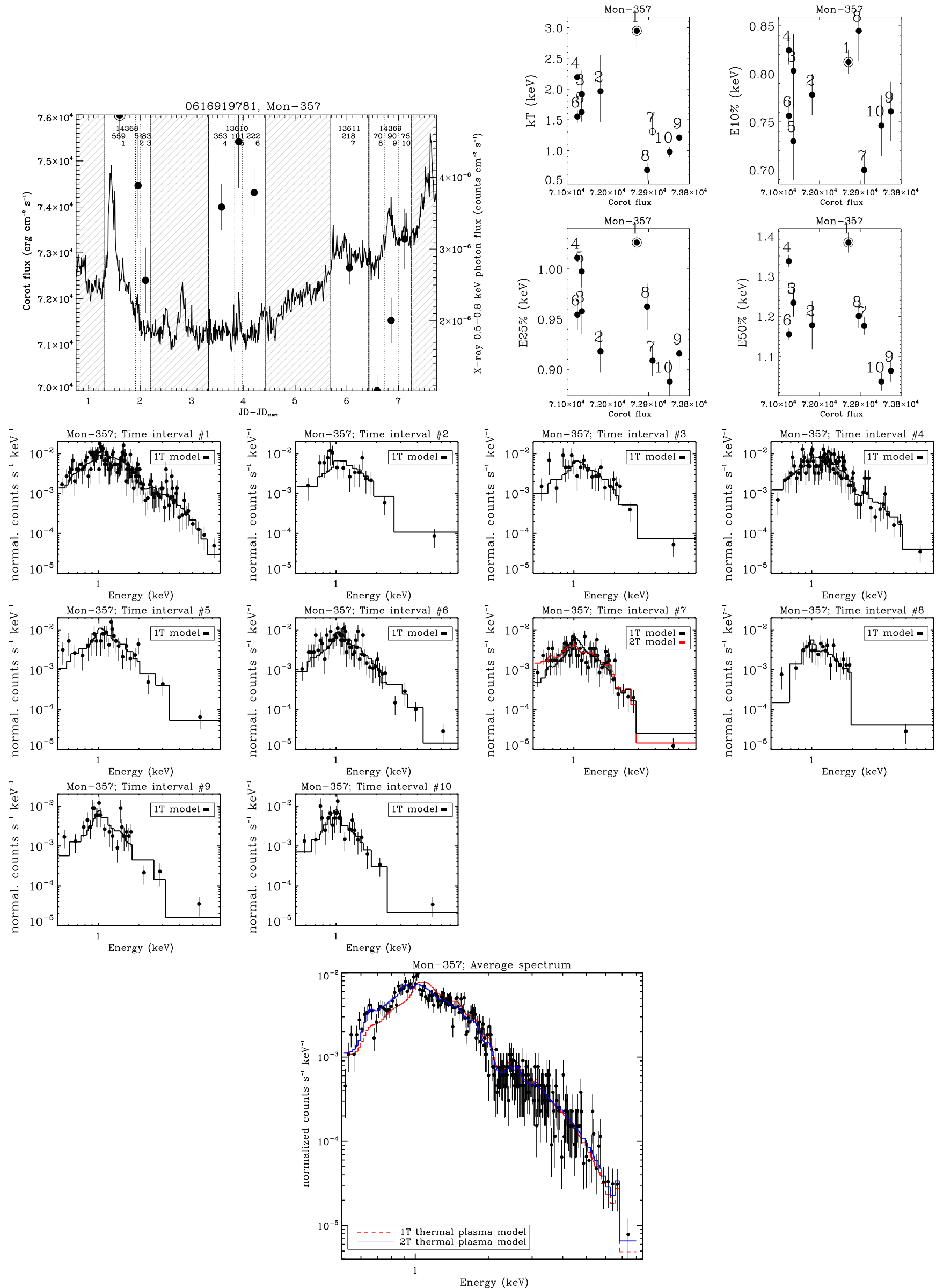

Fig. 17. Time variability of the optical flux and X-ray properties of Mon-357 with panel layout and content as in Fig. 13. 

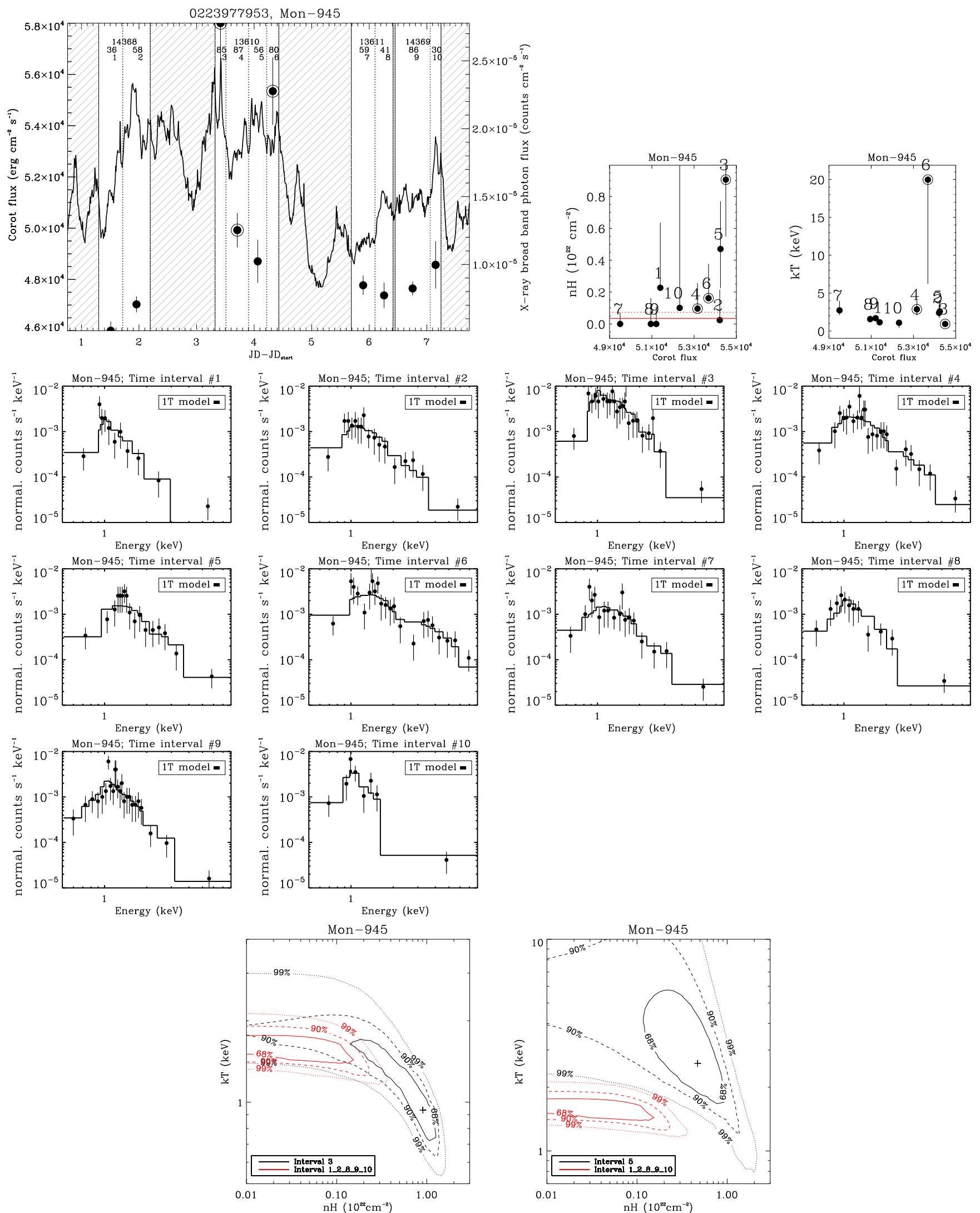

Fig. 18. Time variability of the optical flux and X-ray properties of Mon-945 with panel layout and content as in Fig. 4. Contour levels in the C-stat space corresponding to the $68 \%, 90 \%$, and $99 \%$ statistical confidence regions from the fit of the X-ray spectrum of Mon- 945 observed during the intervals \#3 (bottom left panel, black contours) and \#5 (bottom right panel, black contours) are also shown. In both bottom panels the red contours are obtained from the average spectrum observed in $\# 1+\# 2+\# 8+\# 9+\# 10$. The cross marks the values obtained from the best fit. 

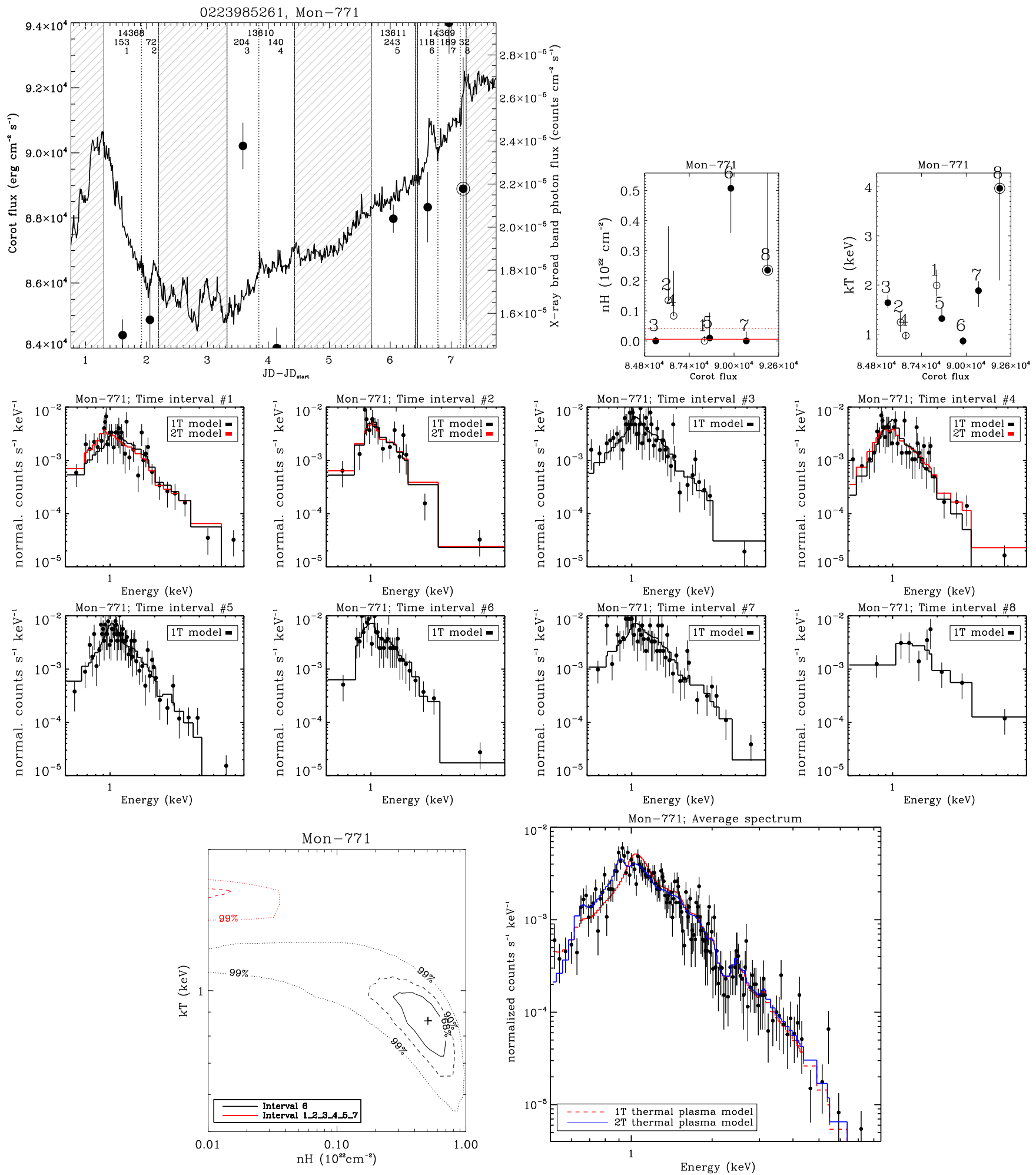

Fig. 19. Time variability of the optical flux and X-ray properties of Mon-771 with the panel format and content as in Fig. 4. Contour levels in the C-stat space corresponding to the $68 \%, 90 \%$, and $99 \%$ statistical confidence regions from the fit of the X-ray spectrum of Mon-771 observed during the time interval \#6 (black contours) are compared to that obtained from the average spectrum observed during the remaining intervals (excluding the flare, red contours). The cross marks the values obtained from the best fit.

CoRoT light curve during the Chandra observations (Fig. 19) is characterized by several small bursts:

- During \#1, the optical emission declines by $5.5 \%$, with a subsequent slow-rising phase longer than the whole Chandra observation.

- An intense optical burst is observed during \#6.
- Small optical bursts are observed during \#2 and \#4.

- The small interval \#8 is dominated by the rising part of an optical and X-ray flare.

- No interesting features are observed during \#3, \#5 and \#7.

The time-resolved X-ray spectra are well fitted by $1 \mathrm{~T}$ thermal plasma models except in \#1, \#2, and \#4. In \#1, an intense 
soft X-ray spectral component between $0.7 \mathrm{keV}$ and $1 \mathrm{keV}$ may be present. The fit with a $2 \mathrm{~T}$ thermal plasma model predicting $N_{\mathrm{H}}=0.21_{-0.21}^{+0.59} \times 10^{22} \mathrm{~cm}^{-2}, k T_{1}=0.31_{-0.16}^{+0.67} \mathrm{keV}$, and $k T_{2}=2.59_{-0.89}^{+3.05} \mathrm{keV}$ is acceptable $\left(P_{\%}=31.5 \%\right)$. Repeating the calculation for the pre-shock velocity, we obtain a value compatible with the free-fall velocity from infinity. Similar results are obtained for \#4, where the spectral fit using the $2 \mathrm{~T}$ thermal plasma model is good $\left(P_{\%}=66.9 \%\right)$; it predicts $N_{\mathrm{H}}=0.0_{-0.0}^{+0.22} \times$ $10^{22} \mathrm{~cm}^{-2}, k T_{1}=0.68_{-0.33}^{+0.20} \mathrm{keV}$, and $k T_{2}=2.69_{-1.34}^{+4.63} \mathrm{keV}$. The spectral fit for the time interval \#2 is poorly constrained even using $2 \mathrm{~T}$ thermal plasma models $\left(P_{\%}=0.3 \%\right)$.

Some evidence of an intense soft X-ray spectral component is shown in the average spectrum (Fig. 19). The fit of the $\mathrm{X}$-ray spectrum with the 1T thermal plasma model is poorly constrained $\left(P_{\%}=0.0\right)$, while an acceptable fit is obtained with the $2 \mathrm{~T}$ thermal model $\left(P_{\%}=13 \%\right)$. This predicts a soft temperature of $k T_{2}=0.86_{-0.02}^{+0.09} \mathrm{keV}$, which is more likely a cool coronal component.

The variability of the X-ray properties during the defined time intervals is shown in the upper right panels of Fig. 19. They suggest that the X-ray absorption is higher in the time interval \#6, dominated by an intense burst. The C-stat contour levels shown in Fig. 19 confirm this conclusion. The $N_{\mathrm{H}}$ obtained during the time interval \#6 is the only one different from zero at $>90 \%$ confidence level, and it is significantly larger than the $\mathrm{X}$-ray absorption obtained from the average spectrum observed in the remaining intervals $\left(N_{\mathrm{H}}=0.0_{-0.0}^{+0.02} \times 10^{22} \mathrm{~cm}^{-2}\right.$, excluding \#8 dominated by a flare).

\subsection{Stars with disks with periodic and quasi-periodic variability}

Periodic optical and X-ray variability can be observed in stars with disks. The emission from accretion hot spots, stellar occultation by circumstellar material, optical darkening due to photospheric dark spots, and enhanced X-ray emission due to coronal active regions can be modulated by stellar rotation. In the sample of NGC 2264 stars with disks observed with CoRoT and Chan$d r a$, six stars show periodic behavior in the CoRoT light curve, and three among them (discussed below) have coherent optical versus X-ray flux variability (Fig. 20).

Mon-765: Mon-765 is a moderately accreting K1 star. The CoRoT light curve (top left panel in Fig. 20) shows periodic variability with a period of $\sim 2.7$ days. The variability of the X-ray properties is shown in the upper right panels. The plasma temperature does not show significant variability with the exception of the interval \#2. The variability of the X-ray and CoRoT fluxes is anticorrelated, as confirmed by a Spearman rank correlation test $(\rho=-0.82, \mathrm{P}(\rho)=0.02)$. Rather than rotational modulation of accretion hot spots or stellar occultation, this behavior is more likely due to rotational modulation of spatially coincident photospheric dark spots and coronal active regions.

Mon-103: the hypothesis made for Mon-765 also holds for Mon-103, a non-accreting K6 star whose variability is assumed by Stauffer et al. (2016) to be due to cold spots. This star has an evacuated inner disk as suggested by its near-infrared colors. The CoRoT light curve of Mon-103 is very regular (central left panel in Fig. 20) and a statistically significant anticorrelation between the optical flux variability and both the X-ray flux and median photon energy variability (respectively, $\rho=-0.89, P(\rho)=0.006$ and $\rho=-0.78, P(\rho)=0.04$, in both cases removing the first three time intervals dominated by flares).
Mon-378: Mon-378 is a K5.5 star with a different behavior from Mon-765 and Mon-103. As reported by Cody et al. (2014), the light curve of this star is likely periodic with superimposed fading episodes probably due to increasing circumstellar extinction. This star has been listed as a star with periodic flux dips in Stauffer et al. (2015). The CoRoT light curve during the four Chandra frames (bottom left panel of Fig. 20) is observed in a decaying phase, which is part of its "periodic" behavior. We do not observe evidence of increasing X-ray absorption during the decline of optical emission, since during all the defined time intervals the X-ray spectral fit admits solutions at low $N_{\mathrm{H}}$ within $68 \%$ confidence. However the lower right panels show that the optical flux variability is correlated with the X-ray flux variability $(\rho=0.94, P(\rho)=0.004)$. This behavior is compatible with the scenario of recurrent occultation of the central star by circumstellar material.

\subsection{Summary of observed simultaneous events}

The total number of stars with disks analyzed in this paper with good detection using CoRoT and Chandra (i.e. Sects. 5.3-5.5 and Appendix C) is 51:

- A total of 24 stars are analyzed as "dippers", that is, they show dips in their CoRoT light curves occurring during the Chandra observations. Among them, in the 7 stars discussed in Sect. 5.3 X-ray absorption increases during the optical dips.

- 20 stars are analyzed as "bursters", that is, their CoRoT light curves show rapid bursts occurring during the Chandra observations. Among them, 5 stars show an intense soft X-ray spectral component typically below $1 \mathrm{keV}$ during the optical bursts, while 2 stars show increasing X-ray absorption during the bursts (Sect. 5.4). It must be noted that 9 stars are analyzed both as "bursters" and "dippers".

- 6 stars have periodic CoRoT light curves.

- 8 stars are not analyzed because: i) they do not show any dominant phenomenon; or ii) X-ray detected photons are too few; or iii) their CoRoT mask is contaminated by nearby bright sources.

It is important to understand whether or not the observed variations of $N_{\mathrm{H}}$ in the time intervals are due to statistical fluctuations rather that real variability. To this aim, we compare the occurrence of time intervals, including flares but excluding intervals with poor X-ray spectral fits, where $N_{\mathrm{H}}$ is larger (within at least $1 \sigma$ significance) than the average absorption estimated by spectral fitting to the average spectrum of each star. For the seven stars discussed in Sect. 5.3, we define 60 time intervals in total, and the X-ray absorption is significantly larger than the individual average value in 13 of those intervals (21.6\% of the cases), and only in two cases (3.3\% of the cases) do the CoRoT light curves do not decline or show dips during the intervals. In the other stars analyzed as "dippers", $N_{\mathrm{H}}$ is larger than the individual average value in 4 time intervals of the 103 defined (3.9\%); in 2 of which, the optical emission decreases. Considering, collectively the stars analyzed as "bursters", those with periodic variability, and those not analyzed, $N_{\mathrm{H}}$ is larger than the individual average value in 17 time intervals over 145 defined (11.7\%), but only in 5 cases $(3.4 \%)$ do the CoRoT light curves clearly not decline or show dips. In these stars, $N_{\mathrm{H}}$ is observed to be variable compared to the individual average value in half of the time intervals with respect the stars analyzed in Sect. 5.3. Considering that stars with bursts and those not analyzed due to there been too few detected X-ray photons can show intrinsically variable 
M. G. Guarcello et al.: CSI 2264: Simultaneous optical and X-ray variability in pre-main sequence stars
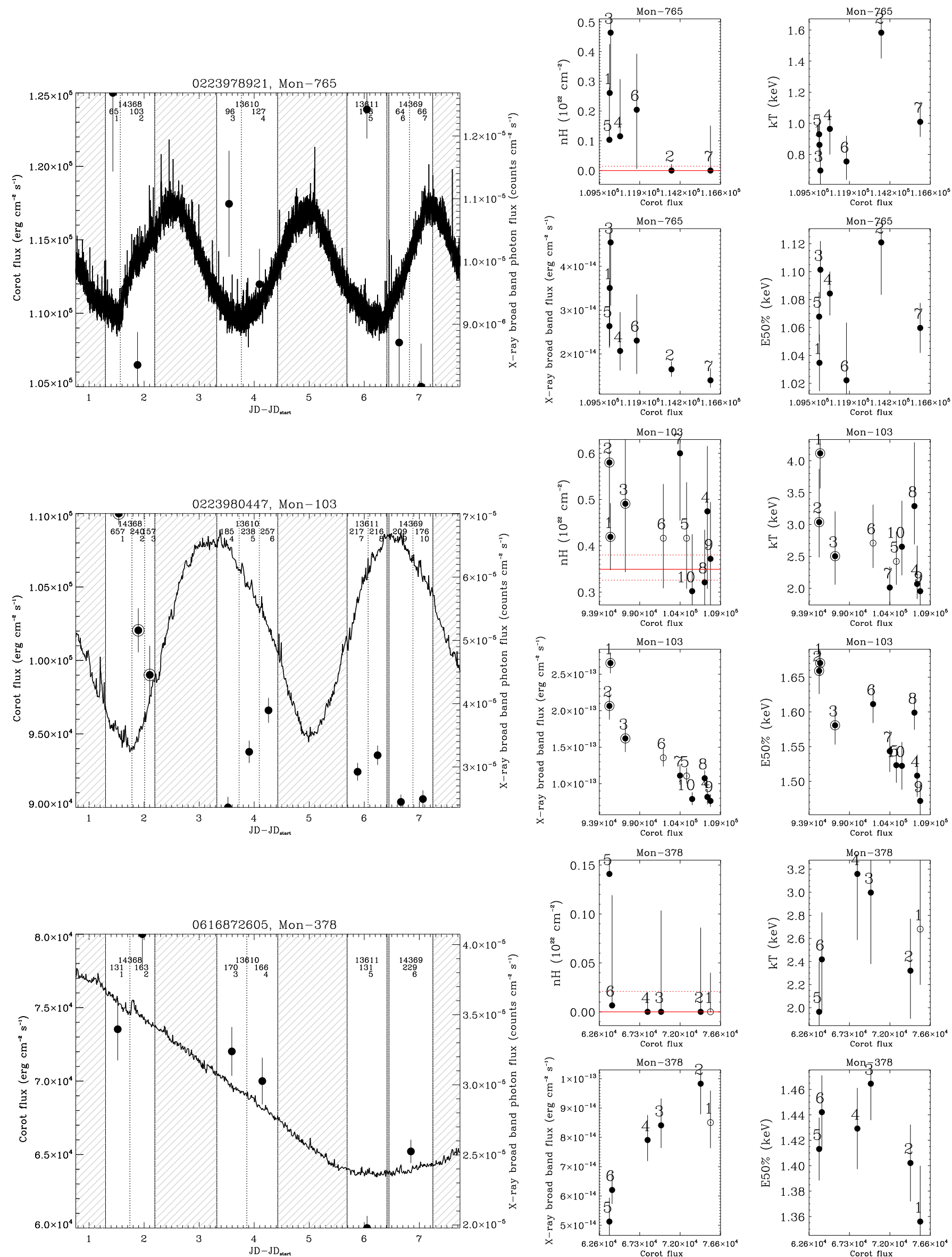

Fig. 20. Time variability of the optical flux and X-ray properties of stars with disks with periodic variability as shown in Fig. 6, with the difference that the X-ray quantities shown in the left panels are: $N_{\mathrm{H}}$ (in units of $10^{22} \mathrm{~cm}^{-2}$ ), $k T$ (in keV), $F_{\mathrm{X}}$ (in erg cm${ }^{-2} \mathrm{~s}^{-1}$ ), and $E_{50 \%}$ in $\mathrm{keV}$. 
X-ray absorption; this strongly suggests that observed variability of X-ray absorption in these stars is not dominated by statistical fluctuations.

It is easier to verify that the observed soft X-ray spectral components below $1 \mathrm{keV}$ in the five stars analyzed in Sect. 5.4 are not due to statistical fluctuations. Such spectral component is in fact observed in 13 time intervals over 43 defined for these stars (occurrence of $30.2 \%$ ). Considering all the other stars analyzed in this paper, we observe 5 possible soft X-ray spectral components below $1 \mathrm{keV}$ (in three cases in stars analyzed as bursters) over 283 time intervals (1.7\% of the cases).

\section{Discussion and conclusions}

In this paper, we analyze the simultaneous variability in optical (from CoRoT) and X-rays (from Chandra/ACIS-I) of stars with disks in NGC 2264, focusing on two samples of stars, those with dips in their CoRoT light curve due to variable extinction, and those with optical bursts due to accretion.

\subsection{The $N_{H} / A_{V}$ ratio during the optical dips}

The hypothesis that stars with disks can be affected by variable extinction due to circumstellar material was first introduced by Joy (1945) and Herbst et al. (1994). If circumstellar material is part of large warps at or inside the co-rotation radius in the disk, then the occultation occurs recurrently over several periods, and the dips are deep. These systems are named "AA Tau like" after star that was the precursor of this class (Bouvier et al. 1999). The CSI 2264 project has also revealed that much shorter and irregular optical dips can be observed in disk-bearing stars. Stauffer et al. (2015) demonstrate that the most likely explanation for narrow and aperiodic dips is the occultation of the central star by dust trapped in unsteady accretion streams, while larger dips are more likely due to disk warps.

However, in those cases where simultaneous increase of optical extinction and X-ray absorption is observed, as discussed in Sect. 5.3, we can attempt to understand the nature of the obscuring material discriminating between dust-free (likely associated with accretion columns) and dust-rich (likely associated with disk warps) material. Two approaches can be adopted.

One method developed by Stauffer et al. (2015) consists of calculating the ratio between the FWHM of the observed dips and the stellar rotation period. The distribution of this ratio is, in fact, observed to be bimodal. Short dips $\left(F W H M_{\text {dip }} / P_{\text {star }} \leq\right.$ 0.25 , where $F W H M_{\text {dip }}$ is the FWHM of the observed dip and $P_{\text {star }}$ the stellar rotation period) are due to occultation by accreting material. Long dips $\left(F W H M_{\text {dip }} / P_{\text {star }}>0.25\right)$ are instead due to occultation by disk warps. We can adopt the rotation periods of NGC 2264 members calculated by Venuti et al. (in prep.) from CoRoT light curves, and calculate the FWHM of the observed dips fitting the dip profile with a Gaussian function.

It is also possible to infer the nature of the obscuring material by calculating the $N_{\mathrm{H}} / A_{V}$ ratio during the dip. Warps are, in fact, located in the inner part of the circumstellar disk, near the co-rotation radius. If the co-rotation radius is larger than the dust sublimation radius, the $N_{\mathrm{H}} / A_{V}$ ratio should be that typical of dust-rich circumstellar disks, assumed to be $1.8-1.9 \times$ $10^{21} \mathrm{~cm}^{-2} \mathrm{mag}^{-1}$ (Burstein \& Heiles 1978; Bohlin et al. 1978). The $N_{\mathrm{H}} / A_{V}$ ratio is expected to be larger in dust-depleted accreting material, since only the small $\sim \mu$ m dust particles can be dragged into the gas streams, sublimating as soon as the temperature reaches $1000-1500 \mathrm{~K}$.
We can infer the $A_{V}$ necessary to reproduce the observed decline of CoRoT flux from the optical flux absorbed during the dip, calculated as the difference between the CoRoT flux observed at the top and at the bottom of the dips, adopting a photometric zero point of $26.6^{m}$ to convert the CoRoT fluxes into $R$ magnitudes, valid for all members brighter than $R \leq 14^{m}$ (Flaccomio et al., in prep.), and the extinction law in $R$ band from Munari \& Carraro (1996). We then compare this with the difference $\Delta N_{\mathrm{H} \text { dip }}$ between the average X-ray spectrum $\left(N_{\mathrm{Haver}}\right)$ and $N_{\mathrm{H}}$ observed during the dip, obtaining $N_{\mathrm{H}} / A_{V}$.

In this calculation, we make a number of assumptions; we assume that both the photosphere and the coronal active regions are obscured by the same structure, or structures with the same composition; we ignore the size of the obscuring structure with respect to the star and we assume that the disk's composition and column density is homogeneous. We also ignore in fact that the inner disk is irradiated by energetic UV and X-ray radiation from the central star and that it may photoevaporate (e.g. Clarke et al. 2001; Pascucci et al. 2011). This process reduces the amount of gas and likely also the amount of small dust particles in the disk, affecting the $N_{\mathrm{H}} / A_{V}$ ratio of the warps.

This calculation is performed over the seven optical dips discussed in Sect. 5.3 where optical extinction and X-ray absorption are observed to increase simultaneously. The physical properties of these dips are summarized in Table 6. In this table, each row corresponds to an observed dip, each star is identified using the "Mon-" name defined by Cody et al. (2014), and we also indicate the time interval containing the observed dip. The CoRoT flux variability during the dip is calculated as $\left(1-\left(F_{\text {low }} / F_{\text {up }}\right)\right) \times 100$, where $F_{\text {low }}$ and $F_{\text {up }}$ are the CoRoT fluxes observed at the bottom and the top of the dip, respectively. Similarly, the variation of the soft X-ray photon flux $\Delta F_{\mathrm{X} \text {,soft }}$ is calculated as $\left(1-\left(F_{\mathrm{X} \text {,pre }} / F_{\mathrm{X} \text {,dip }}\right)\right) \times 100$, where $F_{\mathrm{X} \text {,pre }}$ is the $\mathrm{X}$-ray soft photon flux observed during the time interval before the dip and $F_{\mathrm{X} \text {,dip }}$ during the dip. $P_{\text {star }}, A_{V}, \Delta N_{\mathrm{H} \text { dip }}, N_{\mathrm{H} \text { aver }}$, and the $F W H M_{\text {dip }}$ are obtained as previously explained. In Table 6 , the X-ray properties of the dips observed in Mon-119 are the same since $N_{\mathrm{H}}$ is obtained by combining the X-ray spectra observed in the two dips.

An approximate distinction between dips caused by dustrich and gas-rich material can be made selecting the dips with $N_{\mathrm{H}} / A_{V} \leq 10^{21} \mathrm{~cm}^{-2} \mathrm{mag}^{-1}$ and $N_{\mathrm{H}} / A_{V} \geq 10^{23} \mathrm{~cm}^{-2} \mathrm{mag}^{-1}$, respectively. This classification is shown in Fig. 21, where dips due to material with different gas-to-dust ratios are marked with different symbols. The two points corresponding to Mon-119 show the values measured during the time intervals \#4 and \#6, containing two dips.

Only in one dip (Mon-119, interval \#6) is it the case that the absorbing material may be dust-rich compared to the other dips (but gas-rich compared with the typical interstellar material). In three out of seven dips the absorbing material is likely dustdepleted, as expected in accreting material, while the remaining four have intermediate composition. This is confirmed by the distribution of $N_{\mathrm{H}} / A_{V}$ versus $\mathrm{FWHM}_{\mathrm{dip}} / P_{\text {star }}$, shown in Fig. 22. Symbols mark dips with different gas-to-dust ratios as in Fig. 21. All the dips studied in this paper have $\mathrm{FWHM}_{\text {dip }} / P_{\text {star }} \leq 0.2$, as expected from occultation by narrow accreting columns. In Fig. 22, the anticorrelation between $N_{\mathrm{H}} / A_{V}$ and $\mathrm{FWHM}_{\text {dip }} / P_{\text {star }}$ is not statistically significant, according to a Spearman rank correlation test. However, it is interesting to note that the dips due to the most and least dusty obscuring material (i.e., the highest and lowest $N_{\mathrm{H}} / A_{V}$ ratio), correspond to the largest and the smallest $F W H M_{\text {dip }} / P_{\text {star }}$ ratio, respectively, in agreement with the 
M. G. Guarcello et al.: CSI 2264: Simultaneous optical and X-ray variability in pre-main sequence stars

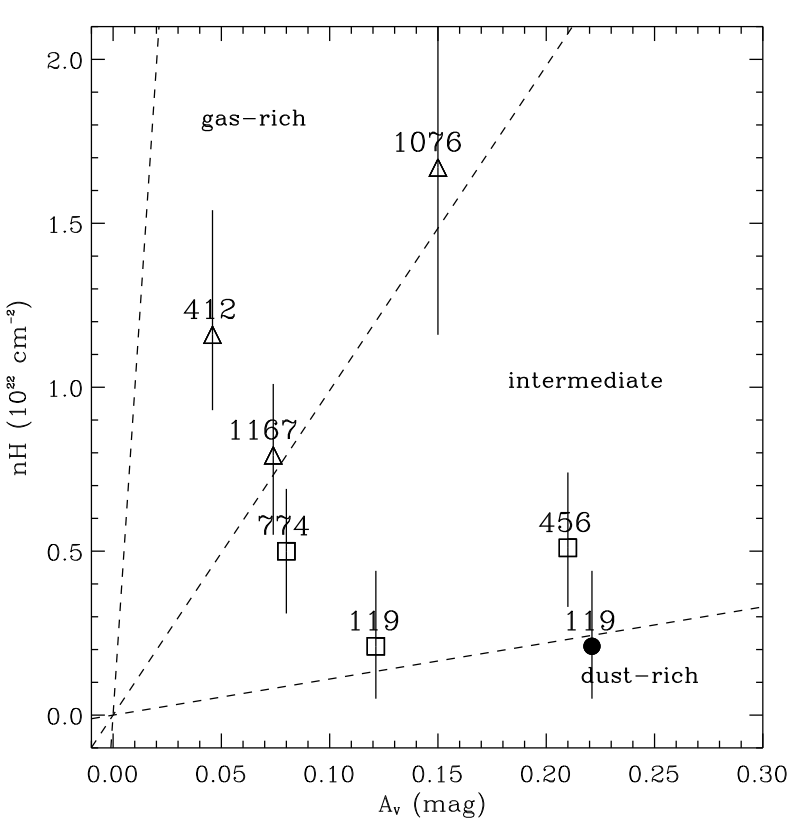

Fig. 21. $N_{\mathrm{H}}$ vs. $A_{V}$ observed in the optical dips listed in Table 6. The lines delimit the three loci populated by dips caused by dust-rich and gas-rich material and with an intermediate gas to dust ratio. The labels indicate the stellar Mon-ID.

hypothesis that dips with larger $F W H M_{\text {dip }} / P_{\text {star }}$ are expected to be produced by dust-rich obscuring material.

The lack of dips due to dust-rich material is very likely a selection effect, since we are studying dips occurring in timescales comparable with the Chandra frames, missing those with larger FWHM $_{\text {dip }}$ which are expected to be produced by disk warps. Additionally, stars occulted by large disk warps are typically faint both in optical and X-rays, so it is not possible to calculate $N_{\mathrm{H}}$ (as in Mon-619 and Mon-717). We are also not particularly sensitive to small variations of $N_{\mathrm{H}}$. Taking all these effects together, we conclude that our results are clearly biased toward narrow dips due to the accretion stream.

\subsection{Global properties of the observed dips}

In the seven dips analyzed in Sect. 6.1, the fraction of absorbed CoRoT flux ranges from $3.5 \%$ (corresponding to an increase of optical extinction by $\left.0.05^{m}\right)$ to $15.7 \%\left(A_{V}\right.$ increasing by $\left.0.22^{m}\right)$, with a mean value of $9.3 \%$ (the largest CoRoT flux variation observed in the 33 stars with variable extinction is $58.1 \%$, corresponding to $A_{V}$ increasing by $1.13^{m}$ ). The FWHM of these seven dips ranges from 0.2 to 2.7 days; six dips are very narrow, with a FWHM ranging from 0.2 to 0.5 days.

More details on the connections between the optical and $\mathrm{X}$-ray variability during these seven dips are shown in Fig. 23. As shown in the left panel, $\Delta N_{\mathrm{Hdip}}$ varies by over a factor of approximately 6 while the $F W H M_{\text {dip }}$ does not change significantly. The only exception is the dip observed in Mon-1076, which has both the largest $F W H M_{\text {dip }}$ and $\Delta N_{\mathrm{H} \text { dip }}$ of the sample. This may suggest that the $F W H M_{\text {dip }}$ is related to the extent of the obscuring material with respect to the stellar disk, and a larger extension does not necessarily imply a larger hydrogen column density.

As expected, there is a correlation between $\Delta N_{\mathrm{H}}$ and $10 \%$ photon energy quantiles observed during the dip (not shown here). A weak correlation is still observed when comparing the $10 \%$ photon energy quantile observed during the dip ( $\left.E_{10 \% \text { dip }}\right)$

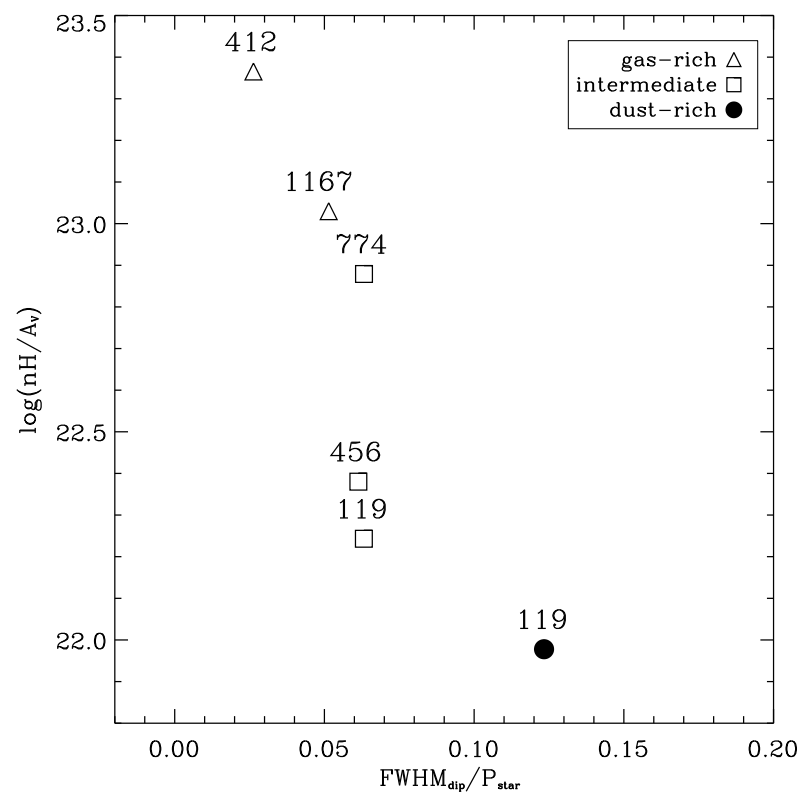

Fig. 22. $\log \left(N_{\mathrm{H}} / A_{V}\right)$ vs. the ratio between the FWHM of the observed dips and the stellar rotation period. The labels indicate the stellar Mon-ID.

with that observed in the average spectrum $\left(E_{10 \% \text { average }}\right)$. A different result is obtained comparing the extinction increment $\Delta A_{V}$

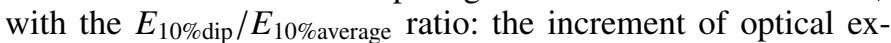
tinction is larger in those dips with small variations of the $10 \%$ photon energy quantile with respect to the average X-ray spectrum. The anticorrelation is significant based on a Spearman rank correlation test.

The direct observation of increasing X-ray absorption during the optical dips is important for two reasons. First, X-rays have an important role in regulating disks evolution. Disk photoevaporation, which is one of the main mechanisms responsible for the dissipation of circumstellar disks (Hollenbach et al. 2000; Alexander et al. 2006) can be induced by incident X-ray photons, which ionize the gas, raising the disk temperature up to $\sim 10^{4} \mathrm{~K}$ within $1 \mathrm{AU}$ of the central star (Ercolano et al. 2008). The resulting high thermal pressure launches a photoevaporation wind, which results in significant mass loss from the disk. Additionally, the more X-ray photons absorbed by the circumstellar disk, the larger the ionization fraction in the disk, and the more efficient the coupling between disk and stellar magnetic field, enhancing magneto-rotational instabilities (Balbus \& Hawley 1991), and thus enhancing the radial transport of gas across the disk. In this context, it is of particular importance to directly observe that stellar X-rays can be efficiently absorbed by the circumstellar material in the inner disk. To date, the only evidence of interaction between energetic particles and protoplanetary disks is the observation of fluorescent emission lines (Tsujimoto et al. 2005). Second, optical and X-ray flux variability is observed to be correlated only in stars with inner disks and/or actively accreting stars (Sect. 4.2 in this paper and Flaccomio et al. 2010). This correlation is interpreted as due to simultaneous occultation of the stellar photosphere and corona by the circumstellar material. Our study supports this hypothesis and provides further evidence of increasing X-ray absorption during the occultation of the central star by circumstellar material. 

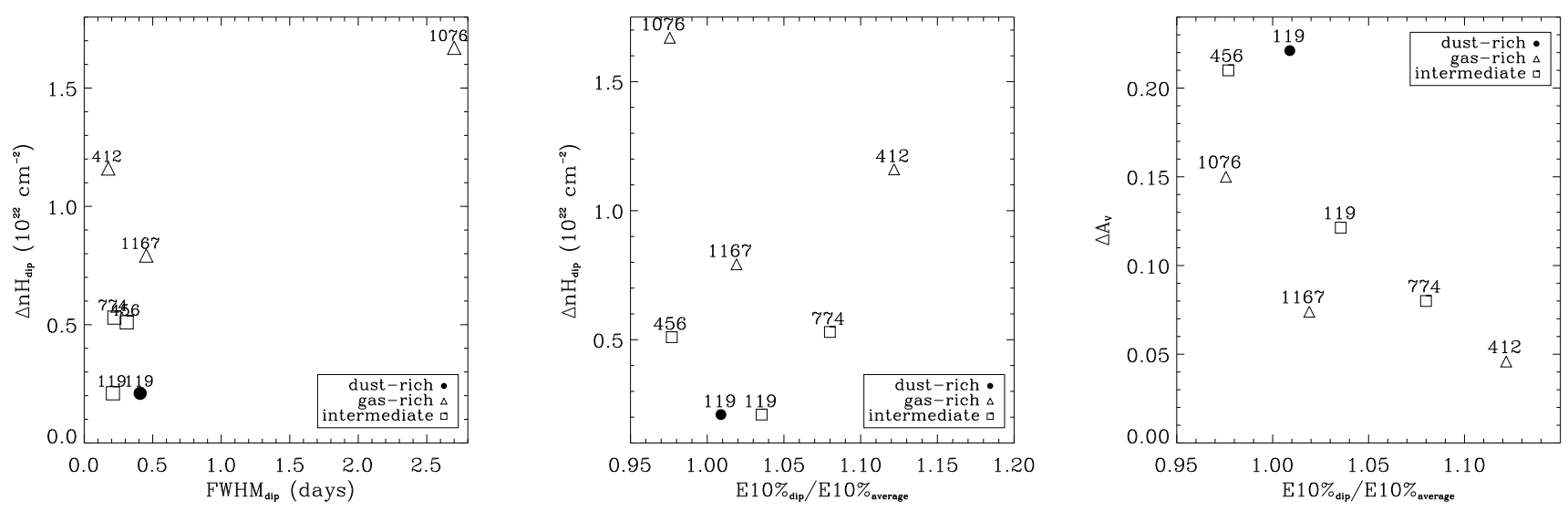

Fig. 23. Optical and X-ray properties of the dips where we observe an increasing X-ray absorption, described in Table 6 . The left panel shows $N_{\mathrm{H}}$ versus FWHM of the dips, the central panel shows $\Delta N_{\mathrm{H}}$ due to the dips versus the ratio between the $10 \%$ photon energy quantile $E_{10 \%}$ observed during the dip and that in the average X-ray spectrum, and the right panel shows $\Delta A_{V}$ increment observed during the dip versus the

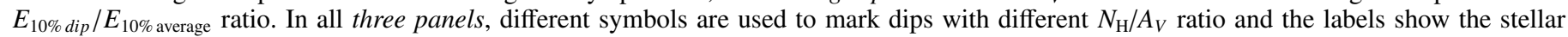
Mon-IDs.

Table 6. Properties of the analyzed optical dips.

\begin{tabular}{ccccccccccc}
\hline \hline Star & Interval & $\Delta F_{\text {CoRoT }}$ & $\Delta A_{V}$ & $\Delta N_{\mathrm{H} \text { dip }}$ & $N_{\mathrm{H} \text { aver }}$ & $N_{\mathrm{H}} / A_{V}$ & $F W H M_{\text {dip }}$ & $P_{\text {star }}$ & $F W H M_{\text {dip }} / P_{\text {star }}$ & $\Delta F_{\mathrm{X}, \text { soft }}$ \\
\hline MON-name & & $\%$ & mag & $10^{22} \mathrm{~cm}^{-2}$ & $10^{22} \mathrm{~cm}^{-2}$ & $10^{22} \mathrm{~cm}^{-2} \mathrm{~m}^{-1}$ & day & day & \\
\hline 119 & 4 & 8.9 & 0.12 & $0.21_{-0.16}^{+0.23}$ & $0^{+0.03}$ & 1.75 & 0.2 & 3.3 & 0.06 & 36.4 \\
119 & 6 & 15.7 & 0.22 & $0.21_{-0.16}^{+0.23}$ & $0^{+0.03}$ & 0.95 & 0.4 & 3.3 & 0.12 & -45.2 \\
412 & 6 & 3.5 & 0.05 & $1.16_{-0.19}^{+0.42}$ & $0.04^{+0.08}$ & 23.2 & 0.2 & 6.8 & 0.03 & 13.7 \\
456 & 6 & 15.2 & 0.21 & $0.51_{-0.18}^{+0.23}$ & $0^{+0.03}$ & 2.4 & 0.3 & 5.1 & 0.06 & 3.7 \\
774 & 5 & 5.7 & 0.08 & $0.53_{-0.22}^{+0.22}$ & $0.54_{-0.24}^{+0.25}$ & 7.57 & 0.2 & 3.5 & 0.06 & 5.9 \\
1076 & 2 & 10.65 & 0.15 & $1.67_{-0.51}^{+0.49}$ & $0^{+0.01}$ & 11.4 & 2.7 & & -7.1 \\
1167 & 3 & 5.5 & 0.07 & $0.79_{-0.24}^{+0.22}$ & $0^{+0.06}$ & 10.7 & 0.5 & 8.8 & 0.05 & 41.3 \\
\hline
\end{tabular}

\subsection{Accretion properties in the stars with optical bursts}

In Sect. 5.4, we analyze the stars showing evidence of increasing soft X-ray emission during the optical bursts. In Fig. 24, we investigate whether such a correlation exists during all the optical bursts and in the X-ray spectra of all the accretors, even when optical bursts are not observed. To this aim, we fit the X-ray spectra of all the non-accreting stars (Class III objects and the Class II objects with passive disks, see Sect. 3.3), and the time-resolved X-ray spectra observed in accreting stars during the optical bursts and in intervals not containing bursts, using $2 \mathrm{~T}$ thermal plasma models. We calculate, then, the ratio between the normalization of the soft and the hard components (Norm.soft/Norm.hard) for each spectrum. The result is shown in Fig. 24, where accreting and non-accreting stars are marked with different symbols, and only the results from statistically significant fits are shown. Even though in Fig. 24 we plot the soft temperatures down to $\sim 0 \mathrm{keV}$, we reiterate that Chandra is not sensitive to very soft $\mathrm{X}$-ray emission, and thus values of $k T_{\text {soft }}$ below $\sim 0.1 \mathrm{keV}$ must not be fully trusted. The first result is that in both panels, Norm.soft/Norm.hard is typically larger in accreting stars than in non-accreting stars. A K-S test in both cases indicates that this difference is significant. While in the bottom panel, all the time-resolved spectra with Norm. .soft/Norm.hard $_{\text {. }} \geq 500$ have $k T \leq 1 \mathrm{keV}$, which cannot be fully trusted; only a small fraction of the time-revolved spectra during the bursts (top panel) have $k T \leq 1 \mathrm{keV}$. This provides evidence for a larger emission measure of cold plasma in accreting stars than in stars with no accretion, and confirms the importance of the time-resolved spectral analysis using the optical light curves as a template to isolate the time intervals with optical bursts occurring.

Our study also sheds some light on the geometry of accretion in the analyzed stars. It is generally accepted that gas accretion from the inner region of circumstellar disks is driven by stellar magnetic field. Several magnetohydrodynamic models (e.g., Kulkarni \& Romanova 2008; Romanova et al. 2013) show that a stellar dipolar magnetic field inclined with respect to the rotation axis produces two stable accretion streams from the inner region of the disk, near the truncation radius, that impact the stellar surface at near free-fall velocity. This is the "stable accretion" scenario, in which the emission from the hot spots (from optical to soft X-rays) is modulated by stellar rotation in a stable and periodic pattern (McKinney et al. 2012; Čemeljić et al. 2013).

Other existing models (i.e., Romanova et al. 2012; Kurosawa \& Romanova 2013) show that accretion can also occur in an unstable regime, when Rayleigh-Taylor instabilities occurring at the disk-magnetosphere boundary result in shortlived accretion streams falling on the stellar surface (see also Colombo et al. 2016). In this case, the optical light curve is dominated by random, short accretion bursts such as those analyzed in Sect. 5.4 and by Stauffer et al. (2014), whose typical duration is of a few hours, smaller than the rotation periods of stars and thus not compatible with stable accretion streams. 
M. G. Guarcello et al.: CSI 2264: Simultaneous optical and X-ray variability in pre-main sequence stars
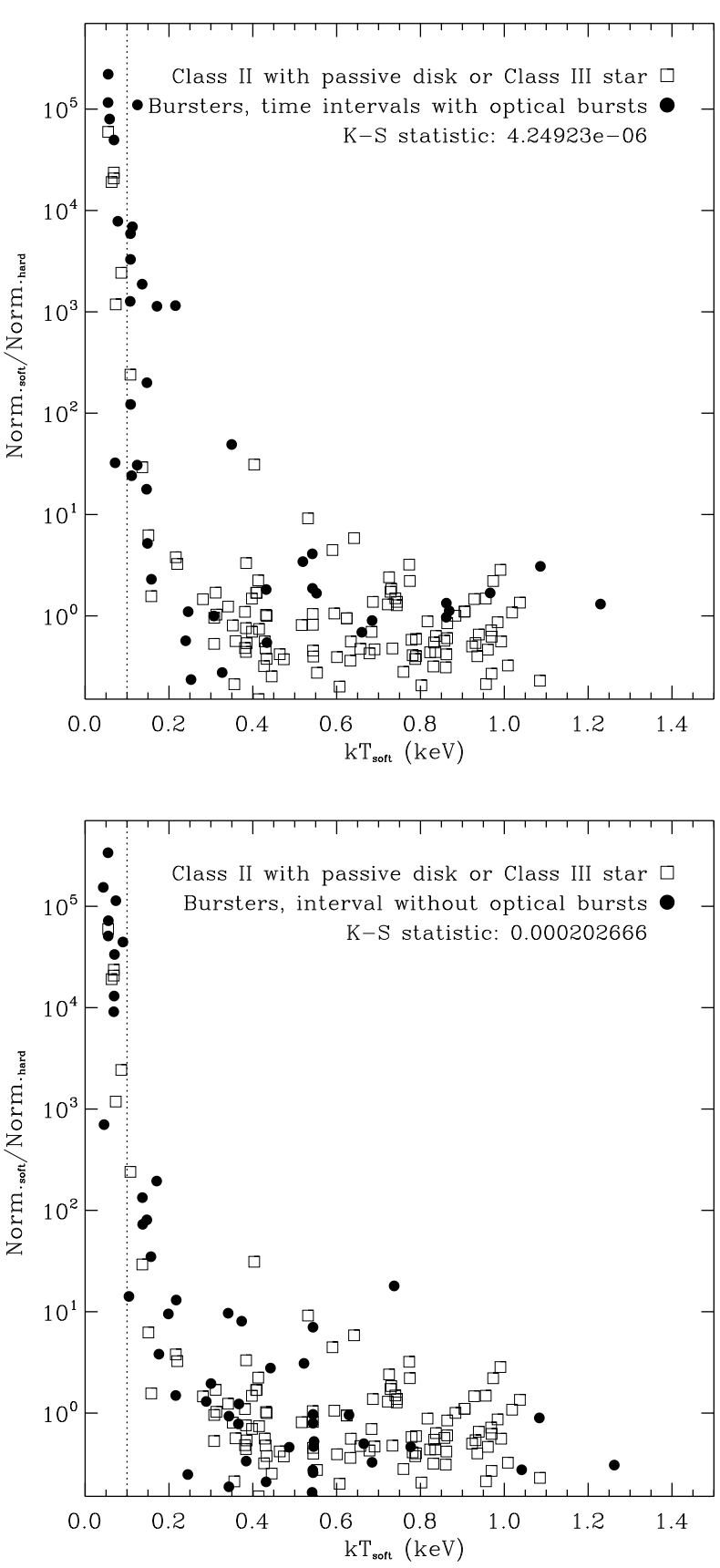

Fig. 24. Ratio between the normalization of the soft and hard components of the best fit $2 \mathrm{~T}$ thermal plasma model vs. the corresponding soft temperature of the X-ray spectra of the non-accreting stars (Class III and Class II objects with passive disks) and that observed in stars with optical bursts in the time intervals with bursts (upper panel) and in those with no bursts (bottom panel).

In some of the stars analyzed in Sect. 5.4, that is, those with an intense soft X-ray spectral component observed during bursts, we can measure the temperature of the plasma responsible for the emission of soft X-rays $\left(k T_{\text {soft }}\right)$ during the bursts, which we assume to be associated with the hot accretion spots. Table 7 shows the values of $k T_{\text {soft }}$ and the properties of the accretion streams obtained in these bursts, the resulting values of the pre-shock velocity and free-fall radius, together with the freefall velocity from infinity and the known $\mathrm{H} \alpha$ equivalent width. The free-fall radius is omitted in those stars where the pre-shock
Table 7. Accretion properties of the stars with soft X-ray emission during the optical bursts.

\begin{tabular}{|c|c|c|c|c|c|c|}
\hline Star & Interval & $k T_{\text {soft }}$ & $v_{\text {presh }}$ & $R_{\mathrm{FF}}$ & $v_{\infty}$ & $E W_{\mathrm{H} \alpha}$ \\
\hline Mon- & \# & $\mathrm{keV}$ & $\mathrm{km} \mathrm{s}^{-1}$ & $R_{\text {star }}$ & $\mathrm{km} \mathrm{s}^{-1}$ & $\AA$ \\
\hline 326 & 1 & $0.15_{-0.08}^{+0.10}$ & $355_{-113}^{+103}$ & & 394 & 27.9 \\
\hline 357 & 7 & $0.06_{-0.02}^{+0.08}$ & & & & \\
\hline 370 & $2,4,7,10$ & $0.16_{-0.04}^{-0.02}$ & $366_{-49}^{+83}$ & $2.0_{-0.4}^{+2.3}$ & 512 & 113.2 \\
\hline 474 & 1 & $0.66_{-0.35}^{+0.16}$ & $744_{-234}^{+85}$ & & 423 & 104.7 \\
\hline 474 & 6 & $0.25_{-0.09}^{+0.10}$ & $458_{-92}^{+84}$ & & 423 & 104.7 \\
\hline 808 & $1,2,8$ & $0.15_{-0.04}^{+0.06}$ & $351_{-47}^{+69}$ & $1.9_{-0.4}^{+2.0}$ & 519 & 50.2 \\
\hline
\end{tabular}

Notes. Typically the co-rotation radius $R_{\text {cor }}$ is $5-10 R_{\text {star }}$ in T Tauri stars. In Mon- $326 R_{\text {cor }}$ is $12.3 R_{\text {star }}$

velocity is not constrained (i.e., when it is compatible with the free-fall velocity from infinity).

In three cases, the soft temperature is approximately $0.15 \mathrm{keV}$ (Mon-326, Mon-370, and Mon-808), with the highest soft temperatures observed in Mon-474 and the lowest in Mon357. The calculated pre-shock velocities vary over a wide range, although being well constrained only in two stars, Mon-370 and Mon-808. In these two cases, $v_{\text {presh }}$ is relatively similar (approximately 350-360 km s${ }^{-1}$ ). In Mon-370 and Mon-808, also the corresponding free-fall radii are also reasonably well constrained, being smaller than the typical co-rotation radii of $\mathrm{T}$ Tauri stars (usually ranging between 5 and $10 R_{\text {star }}$, Hartmann et al. 1998; Shu et al. 2000). Considering that we can safely ignore the energy loss during accretion, and thus that our estimate of the freefall radii is not seriously overestimated, our findings are compatible with the hypothesis that the small accretion bursts observed in these stars and the resulting soft X-ray emission are likely due to unstable accretion rather than stable accretion streams from the co-rotation radius.

Acknowledgements. We thank the referee for his/hers comments and suggestions that helped us to improve our manuscript. M.G.G., E.F. and G.M. acknowledge the grant PRIN-INAF 2012 (P.I. E. Flaccomio). This research has made use of data from the Chandra X-ray Observatory and the CoRoT satellite. This research also made extensive use of Xspec software and the NASA's Astrophysics Data System and Vizier databases, operated at CDS, Strasbourg, France.

\section{References}

Alencar, S. H. P., Teixeira, P. S., Guimarães, M. M., et al. 2010, A\&A, 519, A88 Alexander, R. D., Clarke, C. J., \& Pringle, J. E. 2006, MNRAS, 369, 216 Argiroffi, C., Flaccomio, E., Bouvier, J., et al. 2011, A\&A, 530, A1 Argiroffi, C., Maggio, A., Montmerle, T., et al. 2012, ApJ, 752, 100 Arnaud, K. A. 1996, in Astronomical Data Analysis Software and Systems V, eds. G. H. Jacoby, \& J. Barnes, ASP Conf. Ser., 101, 17

Baglin, A., Auvergne, M., Barge, P., et al. 2006, in The CoRoT Mission PreLaunch Status, Stellar Seismology and Planet Finding, eds. M. Fridlund, A. Baglin, J. Lochard, \& L. Conroy, ESA SP, 1306, 33

Balbus, S. A., \& Hawley, J. F. 1991, ApJ, 376, 214

Barentsen, G., Vink, J. S., Drew, J. E., et al. 2011, MNRAS, 415, 103

Bohlin, R. C., Savage, B. D., \& Drake, J. F. 1978, ApJ, 224, 132

Bonito, R., Orlando, S., Argiroffi, C., et al. 2014, ApJ, 795, L34

Bouvier, J., Chelli, A., Allain, S., et al. 1999, A\&A, 349, 619

Bouvier, J., Grankin, K. N., Alencar, S. H. P., et al. 2003, A\&A, 409, 169

Bouvier, J., Alencar, S. H. P., Boutelier, T., et al. 2007, A\&A, 463, 1017

Broos, P. S., Townsley, L. K., Feigelson, E. D., et al. 2010, ApJ, 714, 1582 Burstein, D., \& Heiles, C. 1978, ApJ, 225, 40

Caramazza, M., Flaccomio, E., Micela, G., et al. 2007, A\&A, 471, 645

Clarke, C. J., Gendrin, A., \& Sotomayor, M. 2001, MNRAS, 328, 485

Cody, A. M., \& Hillenbrand, L. A. 2010, ApJS, 191, 389 
Cody, A. M., Stauffer, J., Baglin, A., et al. 2014, AJ, 147, 82

Colombo, S., Orlando, S., Peres, G., Argiroffi, C., \& Reale, F. 2016, A\&A, 594, A93

Curran, R. L., Argiroffi, C., Sacco, G. G., et al. 2011, A\&A, 526, A104

Dahm, S. E. 2008, in Handbook of Star Forming Region, Vol. I, The Northern Sky, ed. B. Reipurth (San Francisco, CA: ASP), 966

Dahm, S. E., \& Simon, T. 2005, AJ, 129, 829

Damiani, F., Maggio, A., Micela, G., \& Sciortino, S. 1997, ApJ, 483, 350

Damiani, F., Prisinzano, L., Micela, G., \& Sciortino, S. 2006, A\&A, 459, 477

Drew, J. E., Greimel, R., Irwin, M. J., \& Sale, S. E. 2008, MNRAS, 386, 1761

Ercolano, B., Drake, J. J., Raymond, J. C., \& Clarke, C. C. 2008, ApJ, 688, 398

Espaillat, C., Calvet, N., D'Alessio, P., et al. 2007, ApJ, 670, L135

Favata, F., Flaccomio, E., Reale, F., et al. 2005, ApJS, 160, 469

Feigelson, E. D., \& Decampli, W. M. 1981, ApJ, 243, L89

Feigelson, E. D., \& Kriss, G. A. 1981, ApJ, 248, L35

Fürész, G., Hartmann, L. W., Szentgyorgyi, A. H., et al. 2006, ApJ, 648, 1090

Flaccomio, E., Damiani, F., Micela, G., et al. 2003, ApJ, 582, 398

Flaccomio, E., Micela, G., Sciortino, S., et al. 2005, ApJS, 160, 450

Flaccomio, E., Micela, G., \& Sciortino, S. 2006, A\&A, 455, 903

Flaccomio, E., Micela, G., Favata, F., \& Alencar, S. P. H. 2010, A\&A, 516, L8

Flaccomio, E., Micela, G., \& Sciortino, S. 2012, A\&A, 548, A85

Flaherty, K. M., Muzerolle, J., Wolk, S. J., et al. 2014, ApJ, 793, 2

Garmire, G. P., Bautz, M. W., Ford, P. G., Nousek, J. A., \& Ricker, Jr., G. R. 2003 in X-Ray and Gamma-Ray Telescopes and Instruments for Astronomy., eds. J. E. Truemper, \& H. D. Tananbaum, SPIE Conf. Ser., 4851, 28

Getman, K. V., Flaccomio, E., Broos, P. S., et al. 2005, ApJS, 160, 319

Grosso, N., Bouvier, J., Montmerle, T., et al. 2007, A\&A, 475, 607

Guarcello, M. G., Micela, G., Damiani, F., et al. 2009, A\&A, 496, 453

Guarcello, M. G., Drake, J. J., Wright, N. J., et al. 2013, ApJ, 773, 135

Guarcello, M. G., Drake, J. J., Wright, N. J., et al. 2016, ApJS, submitted [arXiv: 1605.01773]

Haisch, Jr., K. E., Lada, E. A., \& Lada, C. J. 2001, ApJ, 553, L153

Hartmann, L., Calvet, N., Gullbring, E., \& D’Alessio, P. 1998, ApJ, 495, 385

Herbst, W., Herbst, D. K., Grossman, E. J., \& Weinstein, D. 1994, AJ, 108, 1906

Hernández, J., Hartmann, L., Megeath, T., et al. 2007, ApJ, 662, 1067

Hollenbach, D. J., Yorke, H. W., \& Johnstone, D. 2000, Protostars and Planets IV (Tucson: University of Arizona Press), 401

Jardine, M., Collier Cameron, A., Donati, J.-F., Gregory, S. G., \& Wood, K. 2006, MNRAS, 367, 917

Joy, A. H. 1945, ApJ, 102, 168

Kastner, J. H., Huenemoerder, D. P., Schulz, N. S., Canizares, C. R., \& Weintraub, D. A. 2002, ApJ, 567, 434

Koenigl, A. 1991, ApJ, 370, L39

Kulkarni, A. K., \& Romanova, M. M. 2008, MNRAS, 386, 673

Kurosawa, R., \& Romanova, M. M. 2013, MNRAS, 431, 2673

Lada, C. J. 1987, in Star Forming Regions, eds. M. Peimbert, \& J. Jugaku, IAU Symp., 115, 1

Lamm, M. H., Bailer-Jones, C. A. L., Mundt, R., Herbst, W., \& Scholz, A. 2004, A\&A, 417, 557

Maggio, A., Flaccomio, E., Favata, F., et al. 2007, ApJ, 660, 1462

Mamajek, E. E. 2009, in AIP Conf. Ser., 1158, eds. T. Usuda, M. Tamura, \& M. Ishii, 3

McGinnis, P. T., Alencar, S. H. P., Guimarães, M. M., et al. 2015, A\&A, 577,
McKinney, J. C., Tchekhovskoy, A., \& Blandford, R. D. 2012, MNRAS, 423, 3083

Ménard, F., Bouvier, J., Dougados, C., Mel'nikov, S. Y., \& Grankin, K. N. 2003, A\&A, 409, 163

Montmerle, T. 1996, in Cool Stars, Stellar Systems, and the Sun, eds. R. Pallavicini, \& A. K. Dupree, ASP Conf. Ser., 109, 405

Morales-Calderón, M., Stauffer, J. R., Hillenbrand, L. A., et al. 2011, ApJ, 733, 50

Munari, U., \& Carraro, G. 1996, A\&A, 314, 108

Muzerolle, J., Allen, L. E., Megeath, S. T., Hernández, J., \& Gutermuth, R. A. 2010, ApJ, 708, 1107

Park, B.-G., Sung, H., Bessell, M. S., \& Kang, Y. H. 2000, AJ, 120, 894

Pascucci, I., Sterzik, M., Alexander, R. D., et al. 2011, ApJ, 736, 13

Ramírez, S. V., Rebull, L., Stauffer, J., et al. 2004, AJ, 127, 2659

Rebull, L. M., Makidon, R. B., Strom, S. E., et al. 2002, AJ, 123, 1528

Robitaille, T. P., Whitney, B. A., Indebetouw, R., \& Wood, K. 2007, ApJS, 169, 328

Romanova, M. M., Ustyugova, G. V., Koldoba, A. V., \& Lovelace, R. V. E. 2012, MNRAS, 421, 63

Romanova, M. M., Ustyugova, G. V., Koldoba, A. V., \& Lovelace, R. V. E. 2013 , MNRAS, 430, 699

Rucinski, S. M., Matthews, J. M., Kuschnig, R., et al. 2008, MNRAS, 391, 1913

Samadi, R., Fialho, F., Costa, J. E. S., et al. 2006, in ESA SP 1306, eds. M. Fridlund, A. Baglin, J. Lochard, \& L. Conroy, 317

Schmitt, J. H. M. M., Robrade, J., Ness, J.-U., Favata, F., \& Stelzer, B. 2005, A\&A, 432, L35

Schwartz, P. R., Thronson, Jr., H. A., Odenwald, S. F., et al. 1985, ApJ, 292, 231

Shu, F. H., Najita, J. R., Shang, H., \& Li, Z.-Y. 2000, Protostars and Planets IV (Tucson: University of Arizona Press), 789

Siess, L., Dufour, E., \& Forestini, M. 2000, A\&A, 358, 593

Smith, R. K., Brickhouse, N. S., Liedahl, D. A., \& Raymond, J. C. 2001, ApJ, 556, L91

Stassun, K. G., van den Berg, M., Feigelson, E., \& Flaccomio, E. 2006, ApJ, 649,914

Stassun, K. G., van den Berg, M., \& Feigelson, E. 2007, ApJ, 660, 704

Stauffer, J., Cody, A. M., Baglin, A., et al. 2014, AJ, 147, 83

Stauffer, J., Cody, A. M., McGinnis, P., et al. 2015, AJ, 149, 130

Stauffer, J., Cody, A. M., Rebull, L., et al. 2016, AJ, 151, 60

Stelzer, B., \& Schmitt, J. H. M. M. 2004, A\&A, 418, 687

Störzer, H., \& Hollenbach, D. 1999, ApJ, 515, 669

Sung, H., Bessell, M. S., Chun, M.-Y., Karimov, R., \& Ibrahimov, M. 2008, AJ, 135,441

Sung, H., Stauffer, J. R., \& Bessell, M. S. 2009, AJ, 138, 1116

Teixeira, P. S., Lada, C. J., Marengo, M., \& Lada, E. A. 2012, A\&A, 540, A83

Testi, L., Birnstiel, T., Ricci, L., et al. 2014, Protostars and Planets VI (Tucson: University of Arizona Press), 339

Tsujimoto, M., Feigelson, E. D., Grosso, N., et al. 2005, ApJS, 160, 503

Čemeljić, M., Shang, H., \& Chiang, T.-Y. 2013, ApJ, 768, 5

Venuti, L., Bouvier, J., Flaccomio, E., et al. 2014, A\&A, 570, A82

Venuti, L., Bouvier, J., Irwin, J., et al. 2015, A\&A, 581, A66

Weisskopf, M. C., Brinkman, B., Canizares, C., et al. 2002, PASP, 114, 1

Wilms, J., Allen, A., \& McCray, R. 2000, ApJ, 542, 914

Wolk, S. J., Rice, T. S., \& Aspin, C. 2013, ApJ, 773, 145 


\section{Appendix A: Time-resolved X-ray spectra \\ of the stars with optical dips discussed \\ in Sect. 5.3}

In this appendix, we show the X-ray spectra observed in the time intervals defined for those stars with dips and with simultaneous increase of X-ray absorption discussed in Sect. 5.3.
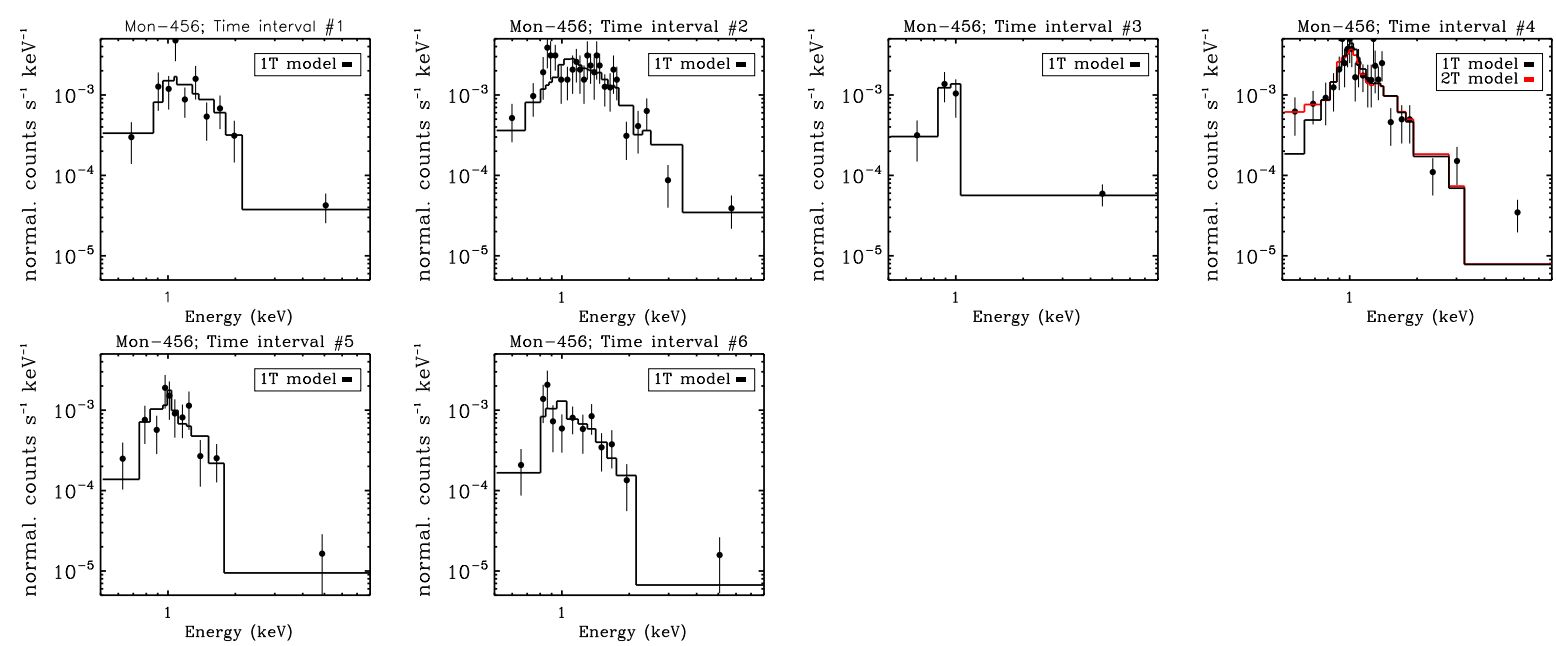

Fig. A.1. X-ray spectra observed in the time intervals defined for Mon-456. In each panel, the spectrum of the fitting model is marked with a solid line, while the black dots mark the observed normalized counts in the given energy bin. X-ray flares occur in \#2 and \#4.
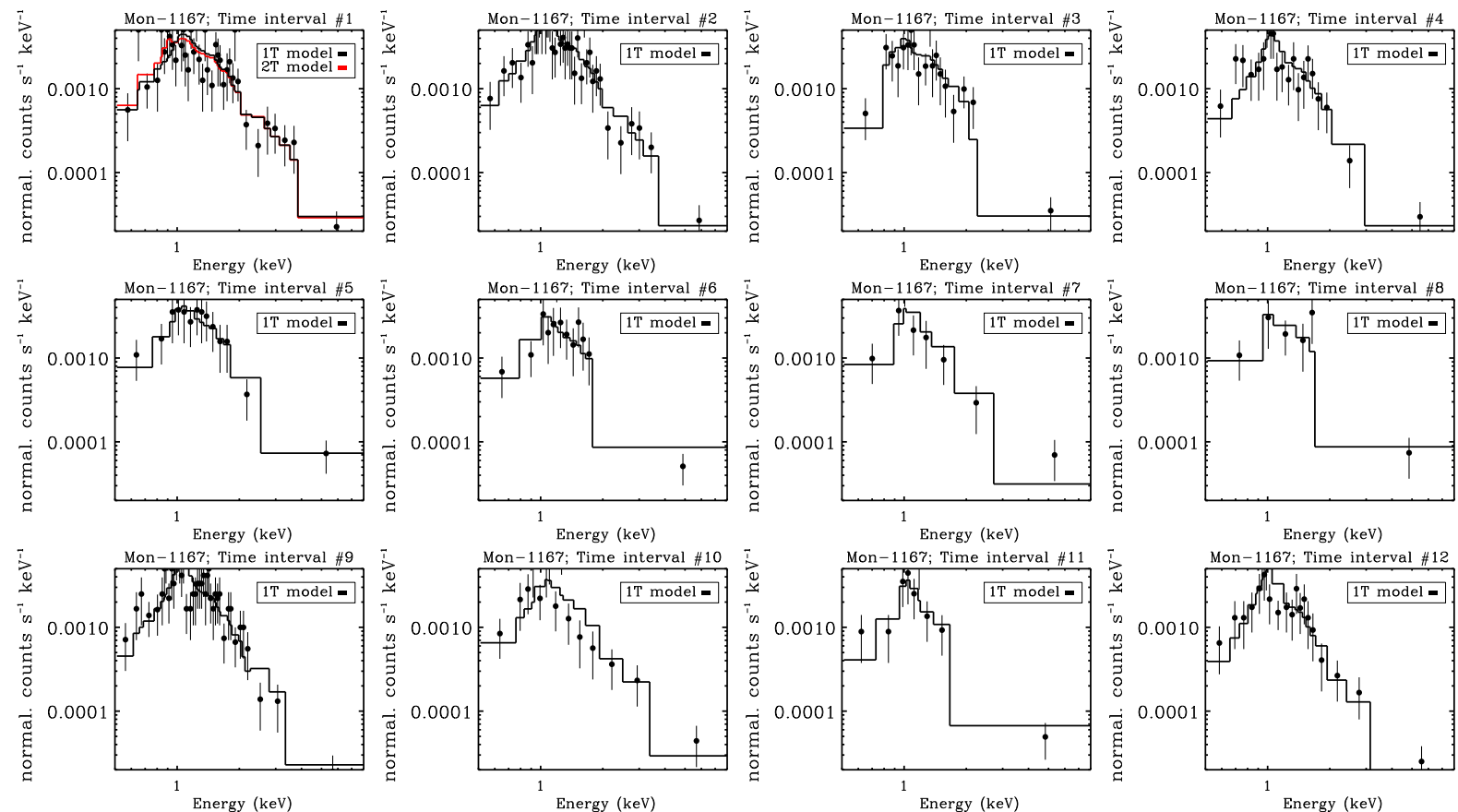

Fig. A.2. X-ray spectra observed in the time intervals defined for Mon-1167. In each panel, the spectrum of the fitting model is marked with a solid line, while the black dots mark the observed normalized counts in the given energy bin. 

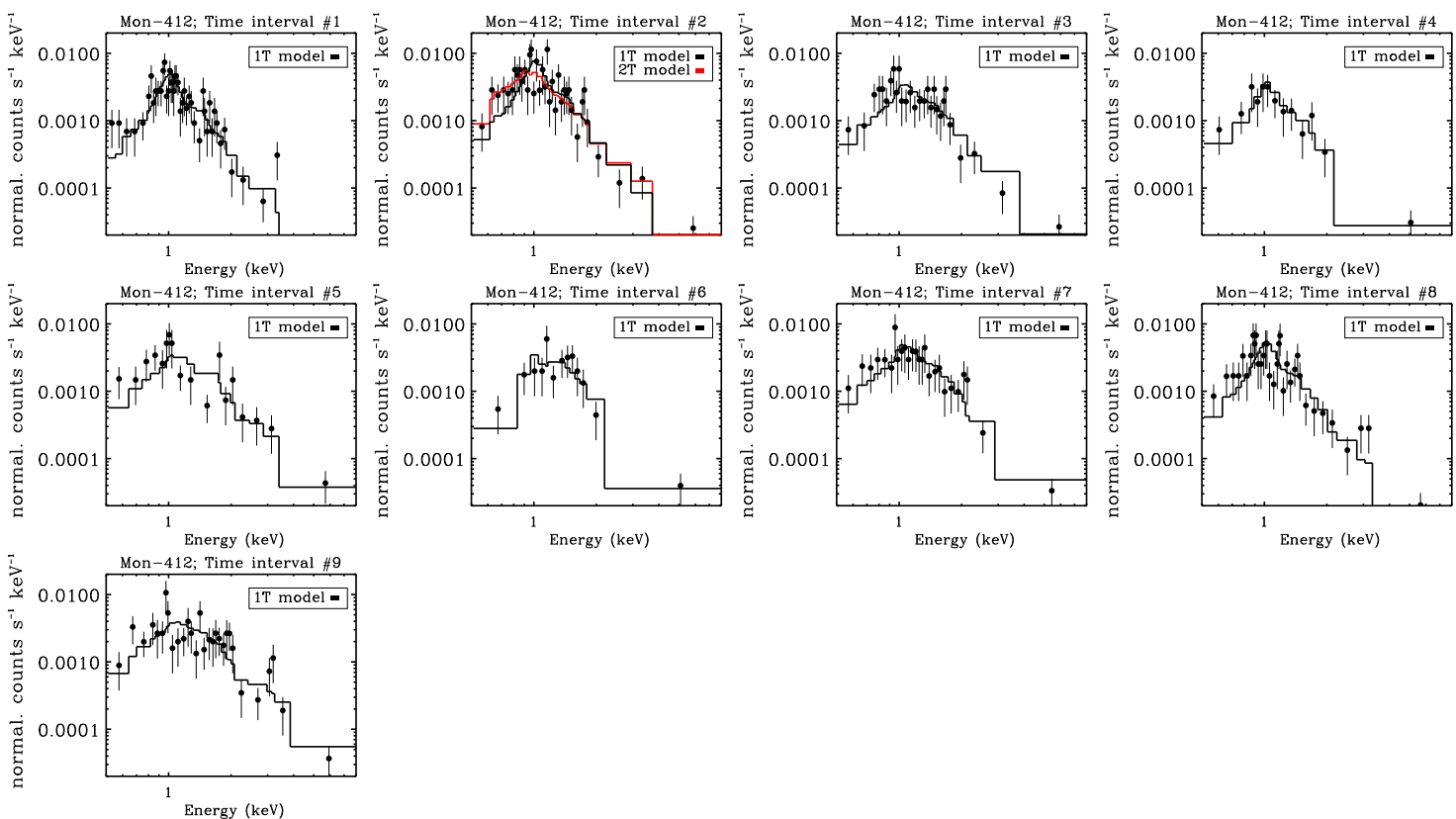

Fig. A.3. X-ray spectra observed in the time intervals defined for Mon-412. In each panel, the spectrum of the fitting model is marked with a solid line, while the black dots mark the observed normalized counts in the given energy bin. This star is a burster and there may be soft X-ray emission related to the bursts, but not significant enough to be analyzed in detail.
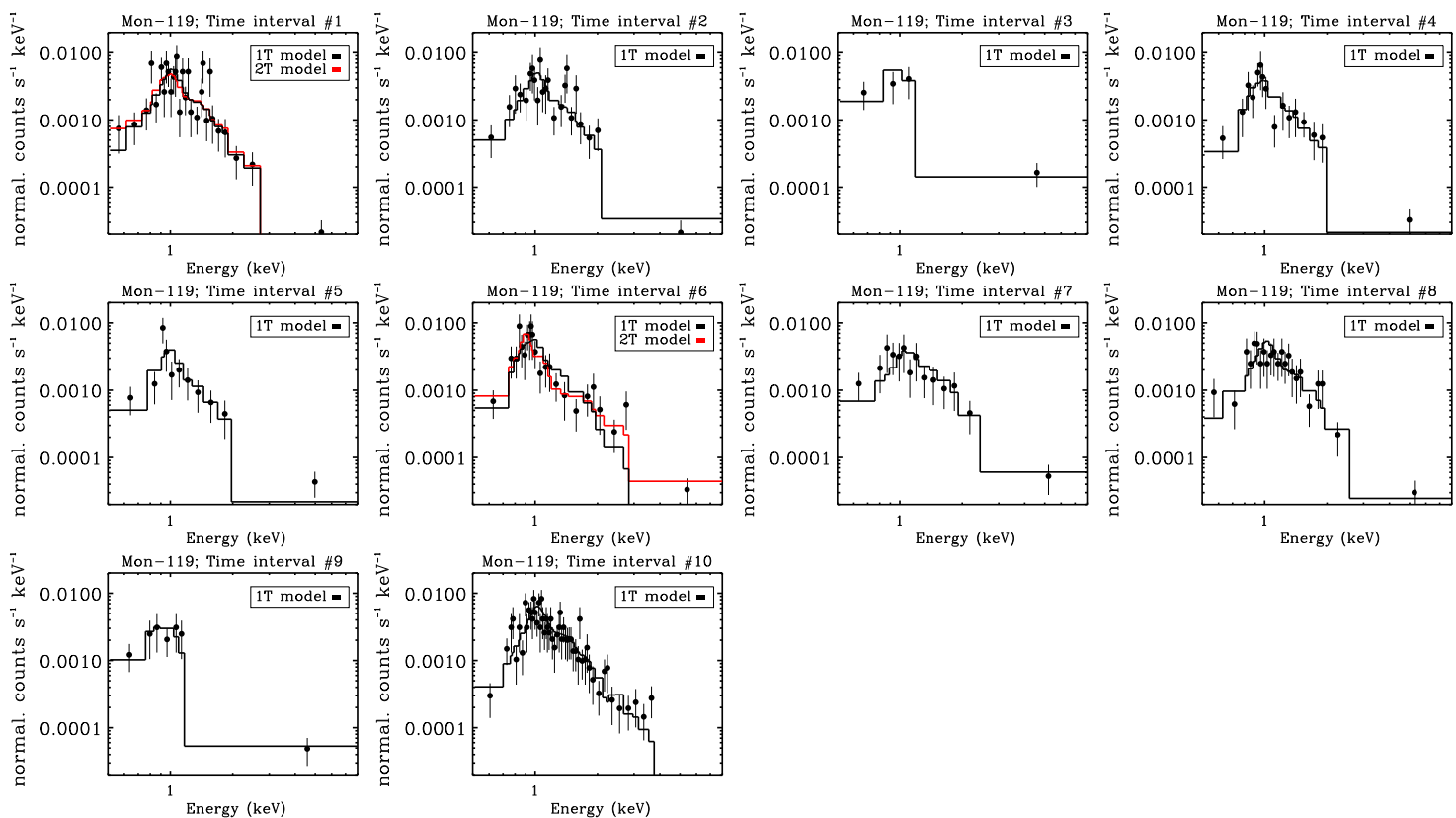

Fig. A.4. X-ray spectra observed in the time intervals defined for Mon-119. In each panel, the spectrum of the fitting model is marked with a solid line, while the black dots mark the observed normalized counts in the given energy bin. An optical and X-ray flare is occurs during \#10. 
M. G. Guarcello et al.: CSI 2264: Simultaneous optical and X-ray variability in pre-main sequence stars
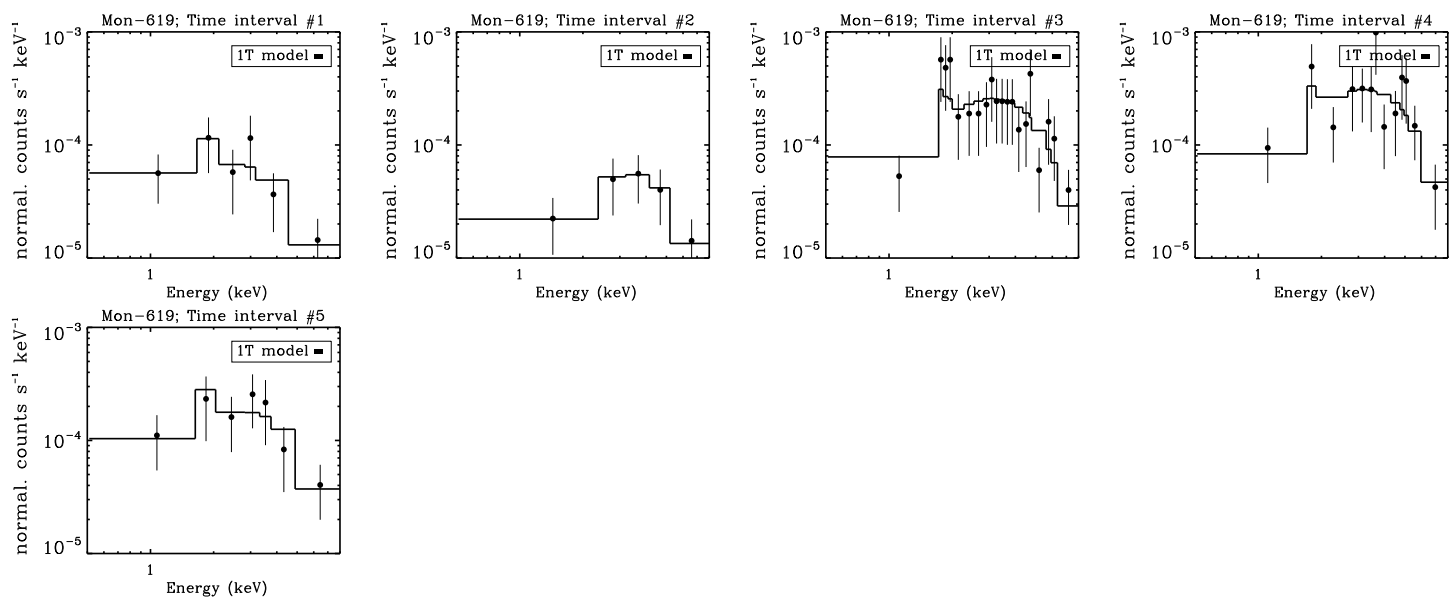

Fig. A.5. X-ray spectra observed in the time intervals defined for Mon-619. In each panel, the spectrum of the fitting model is marked with a solid line, while the black dots mark the observed normalized counts in the given energy bin.
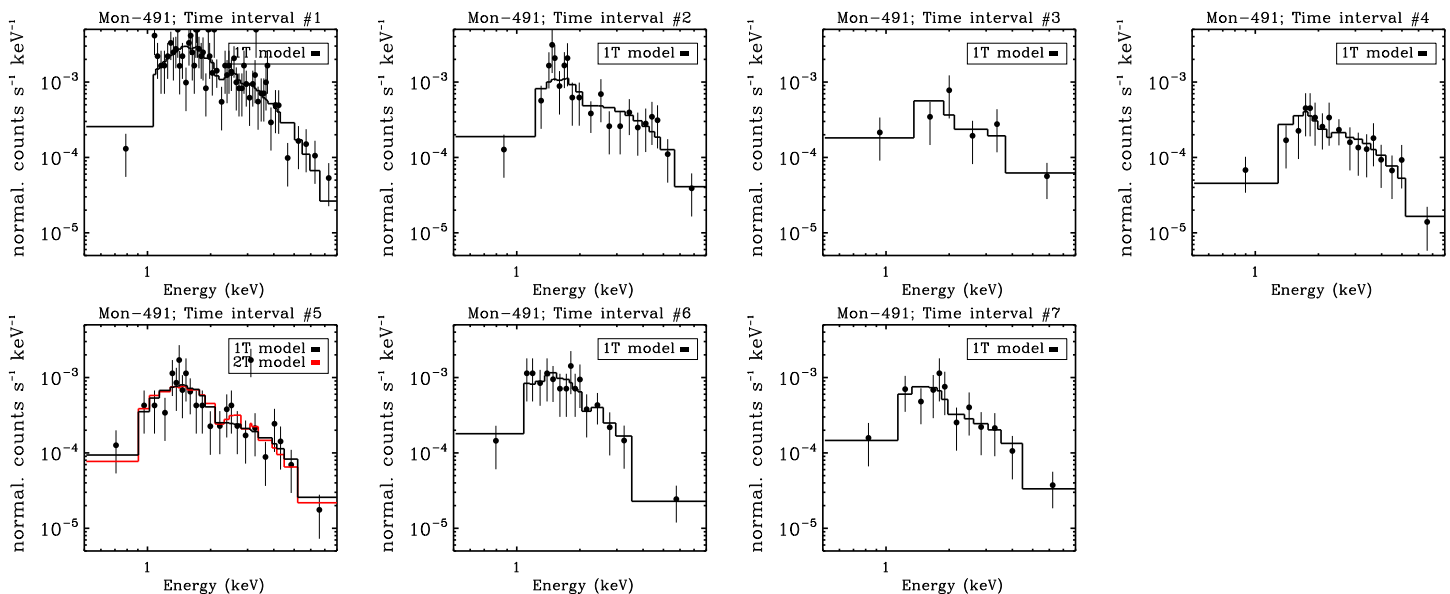

Fig. A.6. X-ray spectra observed in the time intervals defined for Mon-491. In each panel, the spectrum of the fitting model is marked with a solid line, while the black dots mark the observed normalized counts in the given energy bin. An X-ray flare occurs during \#1.
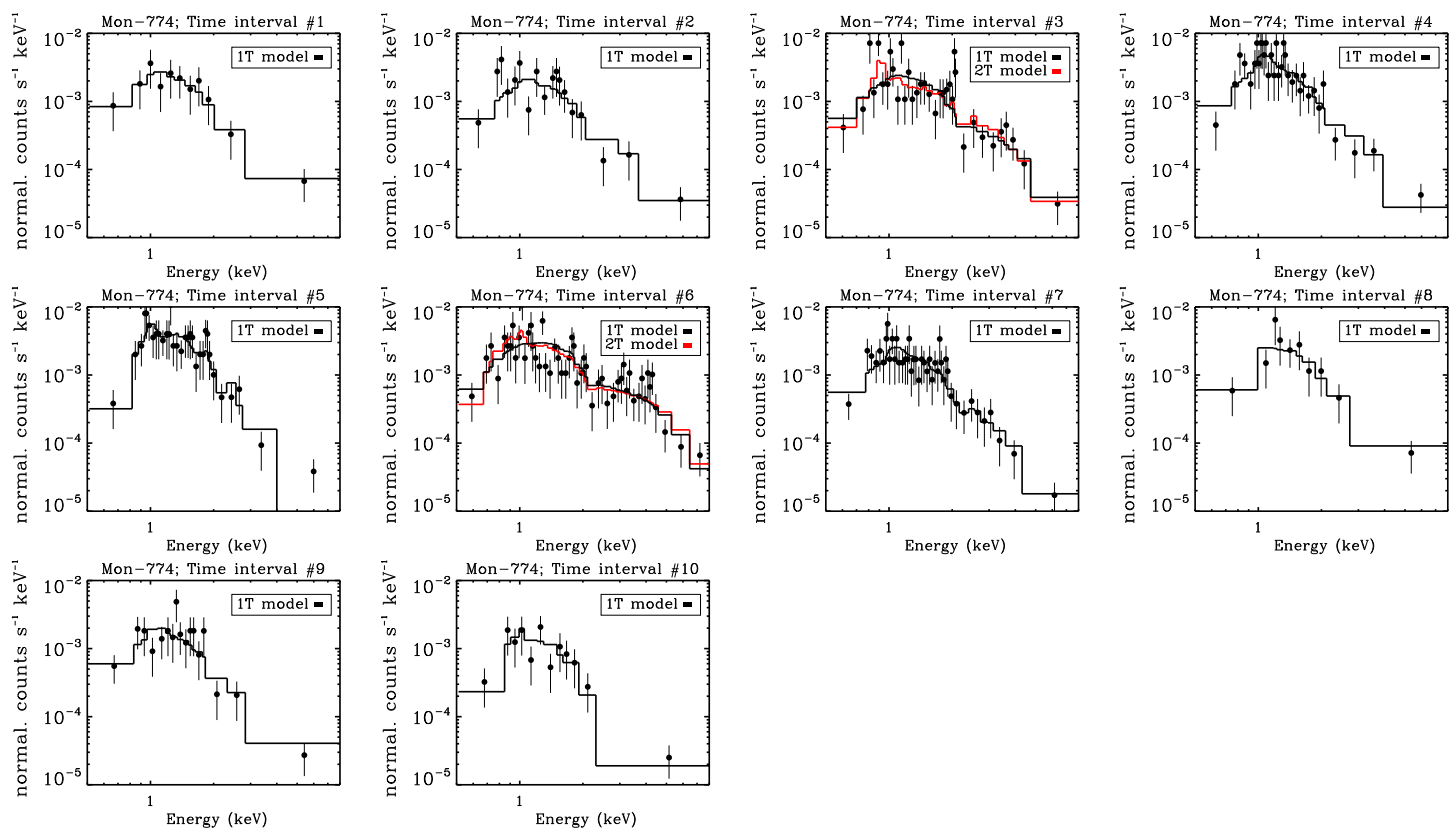

Fig. A.7. X-ray spectra observed in the time intervals defined for Mon-774. In each panel, the spectrum of the fitting model is marked with a solid line, while the black dots mark the observed normalized counts in the given energy bin. A X-ray flare occurs during \#6. 
A\&A 602, A10 (2017)
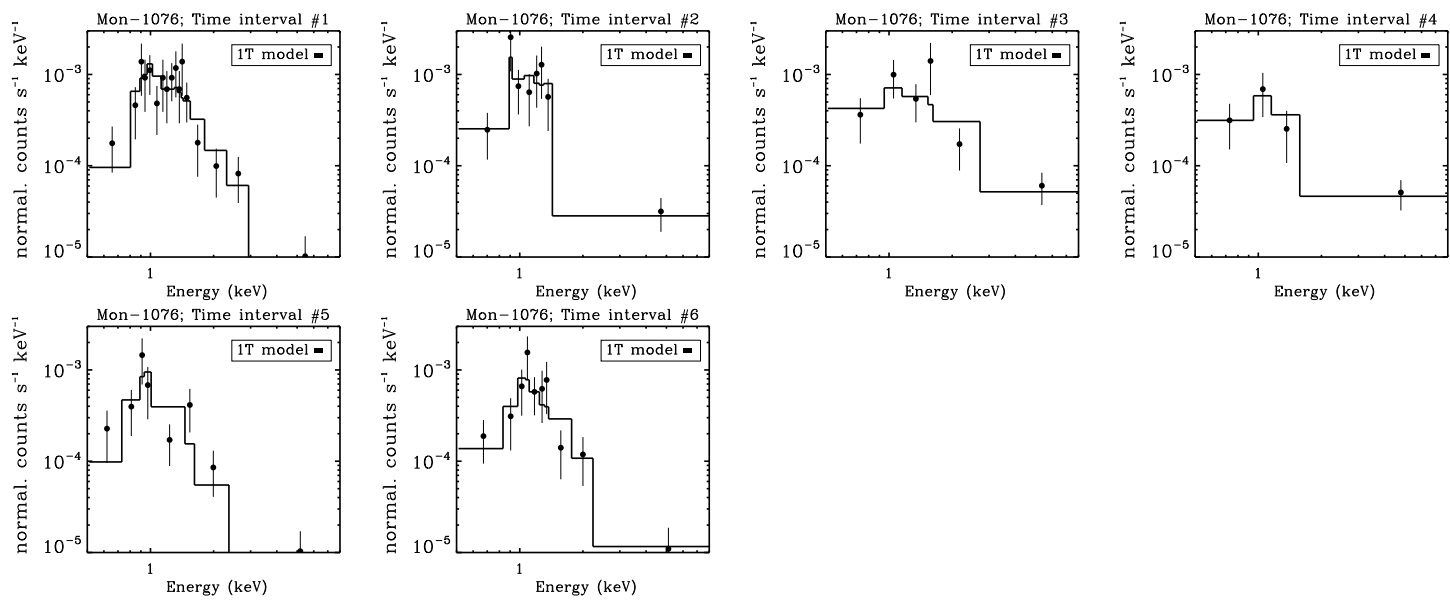

Fig. A.8. X-ray spectra observed in the time intervals defined for Mon-1076. In each panel, the spectrum of the fitting model is marked with a solid line, while the black dots mark the observed normalized counts in the given energy bin. An optical and X-ray flare occurs during \#3. 
M. G. Guarcello et al.: CSI 2264: Simultaneous optical and X-ray variability in pre-main sequence stars

\section{Appendix B: Time variability of the $10 \%$ photon energy quantile in stars discussed in the paper}

In this appendix, we show how the $10 \%$ photon energy quantile changes with time in the stars discussed in the paper. For each star, the time bin used to sample the variability of $E_{10 \%}$ is the shortest one resulting in a relative error $\sigma\left(E_{10 \%}\right) / E_{10 \%}$ smaller than the threshold shown in the title of each panel. The values of $E_{10 \%}$ are marked with blue dots and the red lines indicate the size of the time bin. In each panel, we also show the observed CoRoT light curve, observed during the Chandra frames with the time intervals dominated by X-ray flares covered in black. These figures help us to understand how the soft X-ray spectrum of these stars changes over time independently from the time sampling based on the optical variability. (We note that the larger the $N_{\mathrm{H}}$, the larger $E_{10 \%}$, while the larger flux below $1 \mathrm{keV}$, the lower $E_{10 \%}$.) 
A\&A 602, A10 (2017)
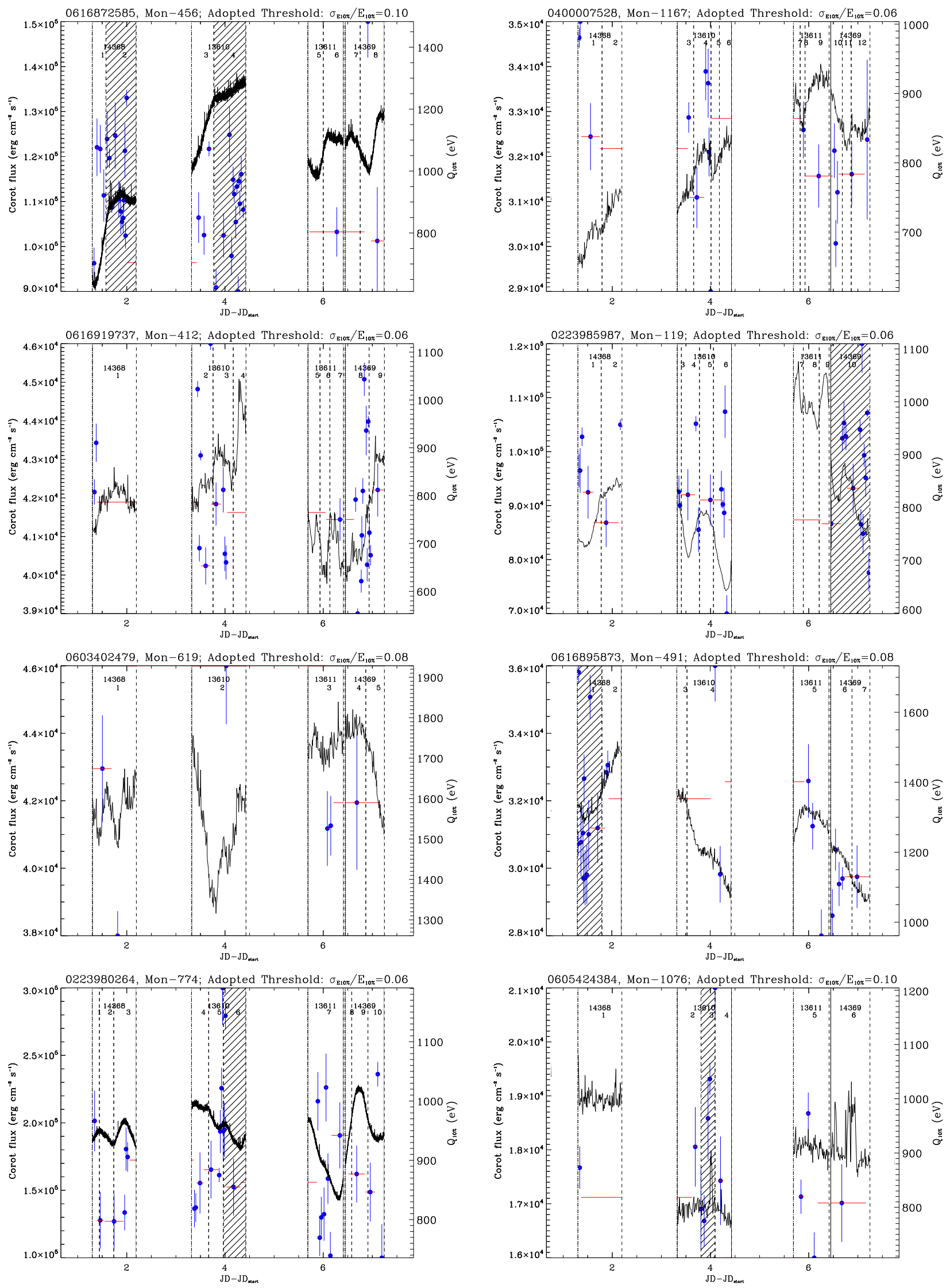

Fig. B.1. CoRoT light curves during the Chandra observations of the stars discussed in the paper, with the time variability of the $10 \%$ photon energy quantile (blue dots). 
M. G. Guarcello et al.: CSI 2264: Simultaneous optical and X-ray variability in pre-main sequence stars
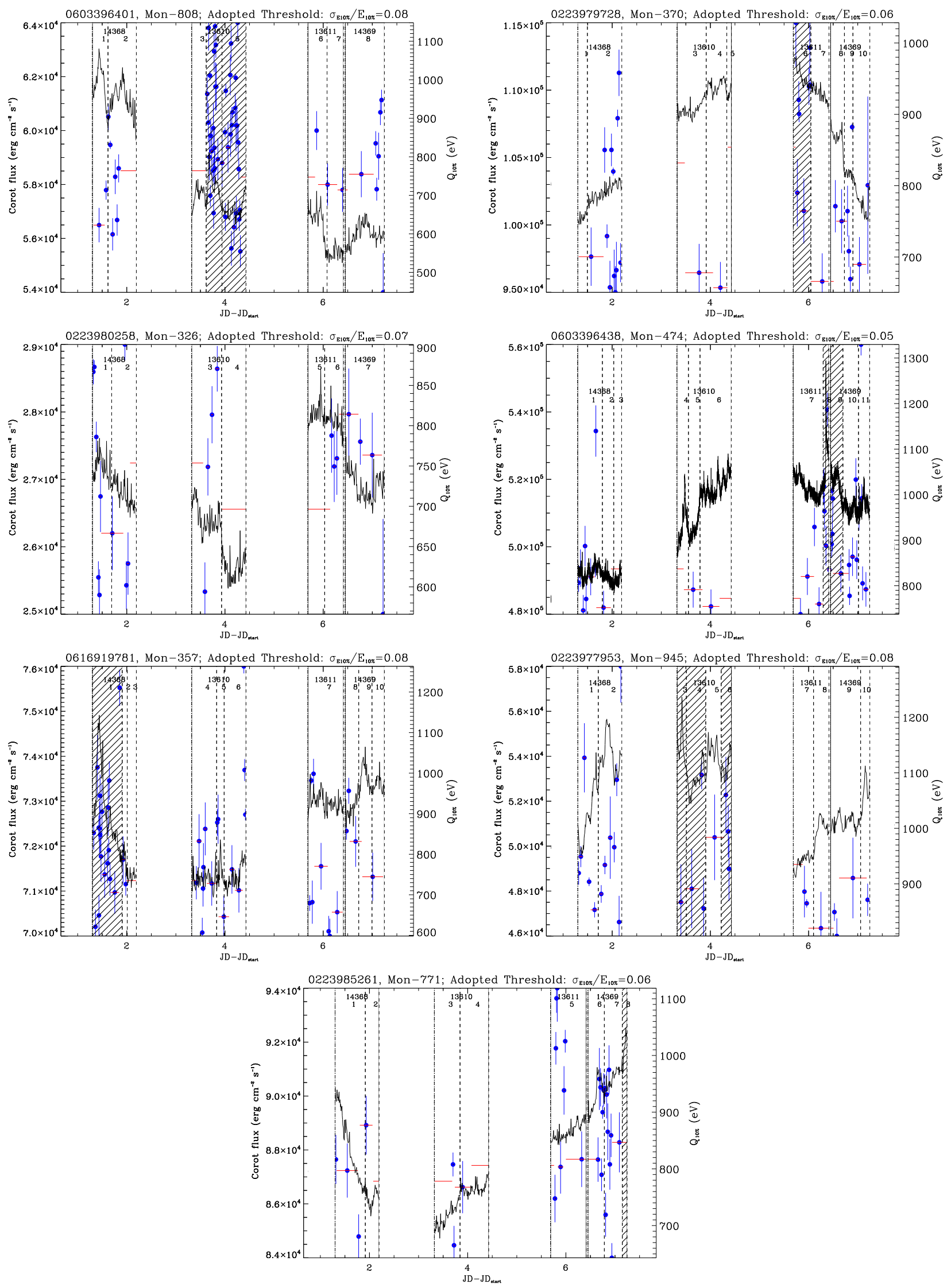

Fig. B.1. continued. 


\section{Appendix C: Optical and X-ray light variability of disk-bearing stars not discussed in the paper}

In this appendix, we show the optical and X-ray variability and the time-resolved X-ray spectra of those stars with disks observed with CoRoT and Chandra that have not been discussed in the paper for the reasons explained in each caption. For each star, we show the CoRoT light curve observed during the four Chan$d r a$ frames; the X-ray spectra observed in the time intervals; and the variability of the following X-ray properties: $N_{\mathrm{H}}$ (in units of $10^{22} \mathrm{~cm}^{-2}$ ), and $k T$ (in $\mathrm{keV}$ ), together with that of the $10 \%$ and $25 \%$ photon energy quantiles (in $\mathrm{keV}$ ) for the stars analyzed as "dippers"; $k T$ and the $10 \%, 25 \%$, and $50 \%$ photon energy quantiles for the stars analyzed as "bursters"; $N_{\mathrm{H}}, k T, F_{\mathrm{X}}$ (in erg $\mathrm{cm}^{-2} \mathrm{~s}^{-1}$ ), and the median photon energy for the stars analyzed as periodic or quasi-periodic variable stars. We also show the variability of $E_{10 \%}$ as in Appendix B.
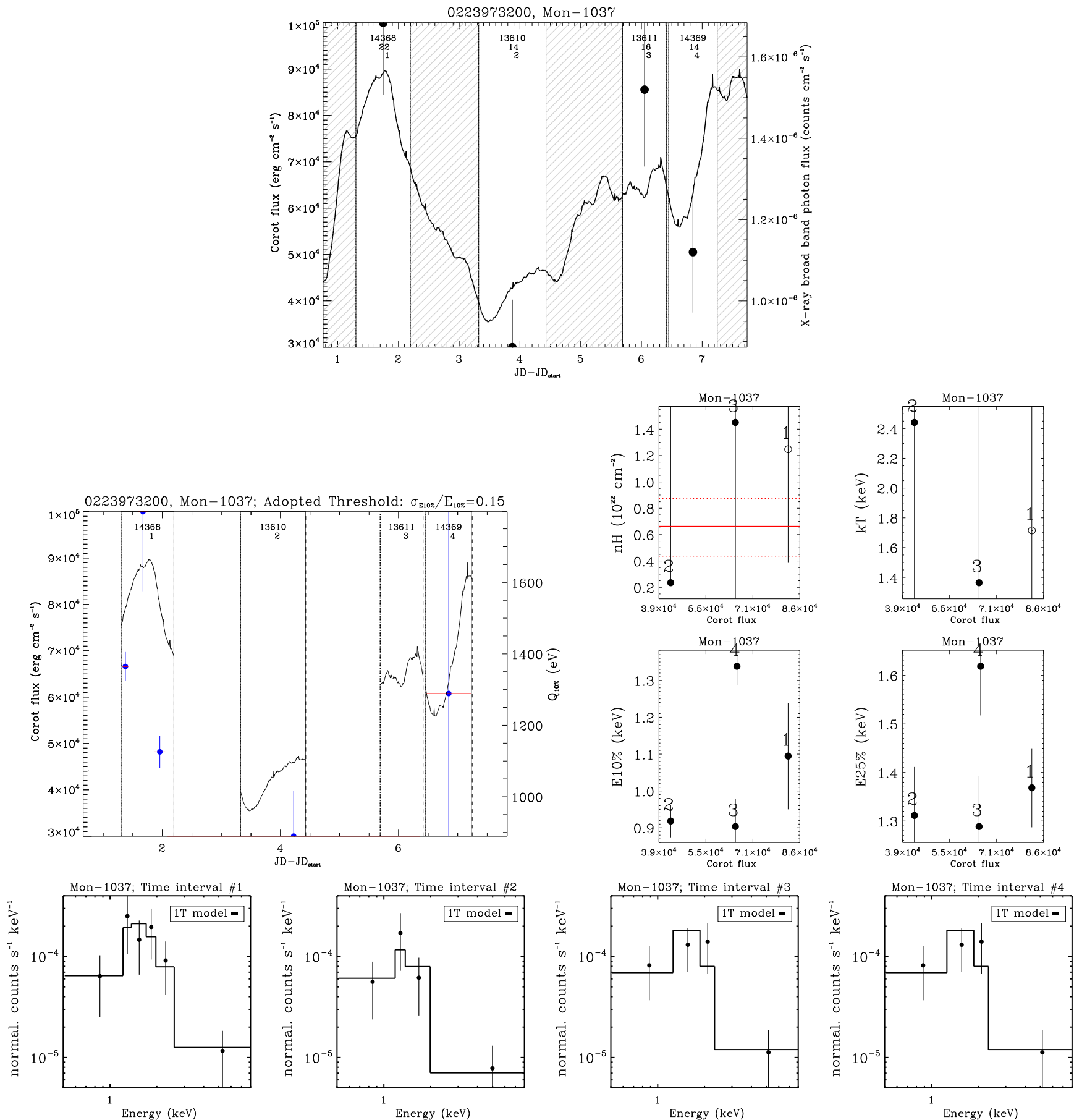

Fig. C.1. Variability and X-ray spectra of Mon-1037, analyzed as a dipper. $E_{10 \%}$ is larger during the dip in \#4, but a significant variability of $N_{\mathrm{H}}$ is not observed. 
M. G. Guarcello et al.: CSI 2264: Simultaneous optical and X-ray variability in pre-main sequence stars
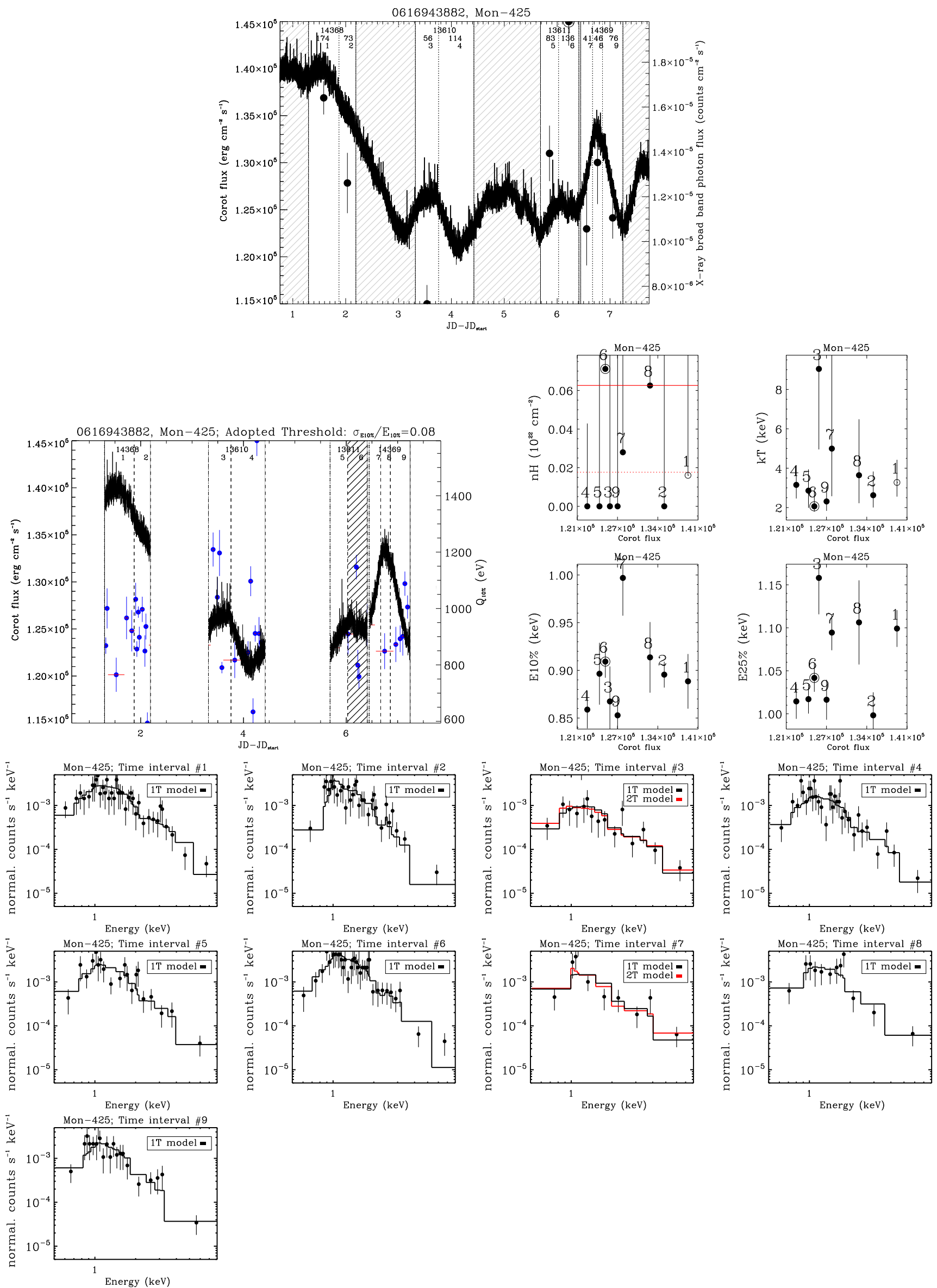

Fig. C.2. Variability and X-ray spectra of Mon-425, analyzed as a dipper. $E_{10 \%}$ is larger during the dip in \#7, but there are no other significant correlations between the optical and X-ray variability. 

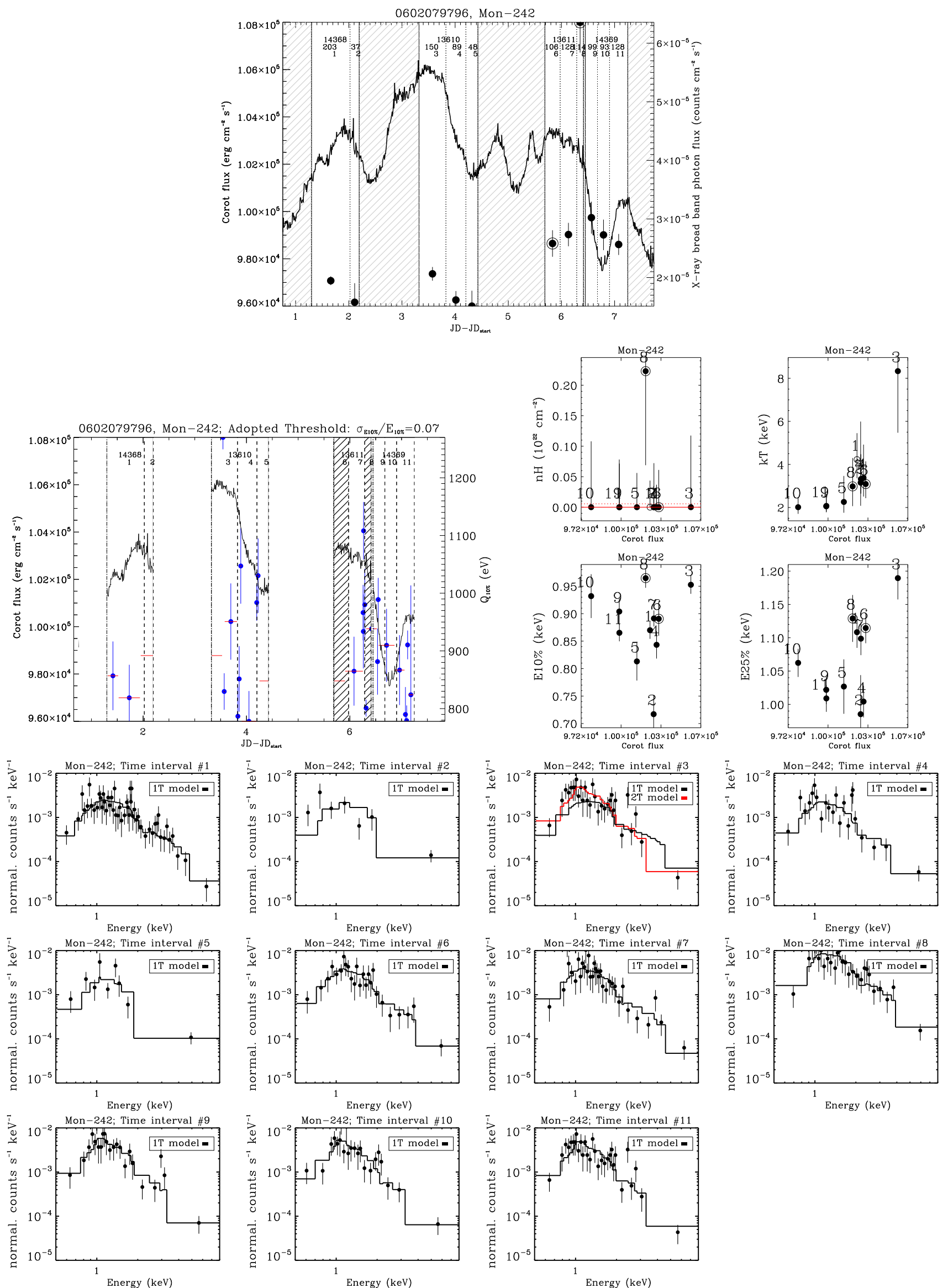

Fig. C.3. Variability and X-ray spectra of Mon-242, analyzed as a dipper. The CoRoT light curve shows several dips without evidence for increasing $N_{\mathrm{H}}$. The spectrum in \#2 is very soft, but the low X-ray count rate does not allow us to verify the presence of a soft X-ray spectral component. The spectral fit with $2 \mathrm{~T}$ thermal plasma model in \#3 is poorly constrained $\left(P_{\%}=0.0\right)$. 
M. G. Guarcello et al.: CSI 2264: Simultaneous optical and X-ray variability in pre-main sequence stars
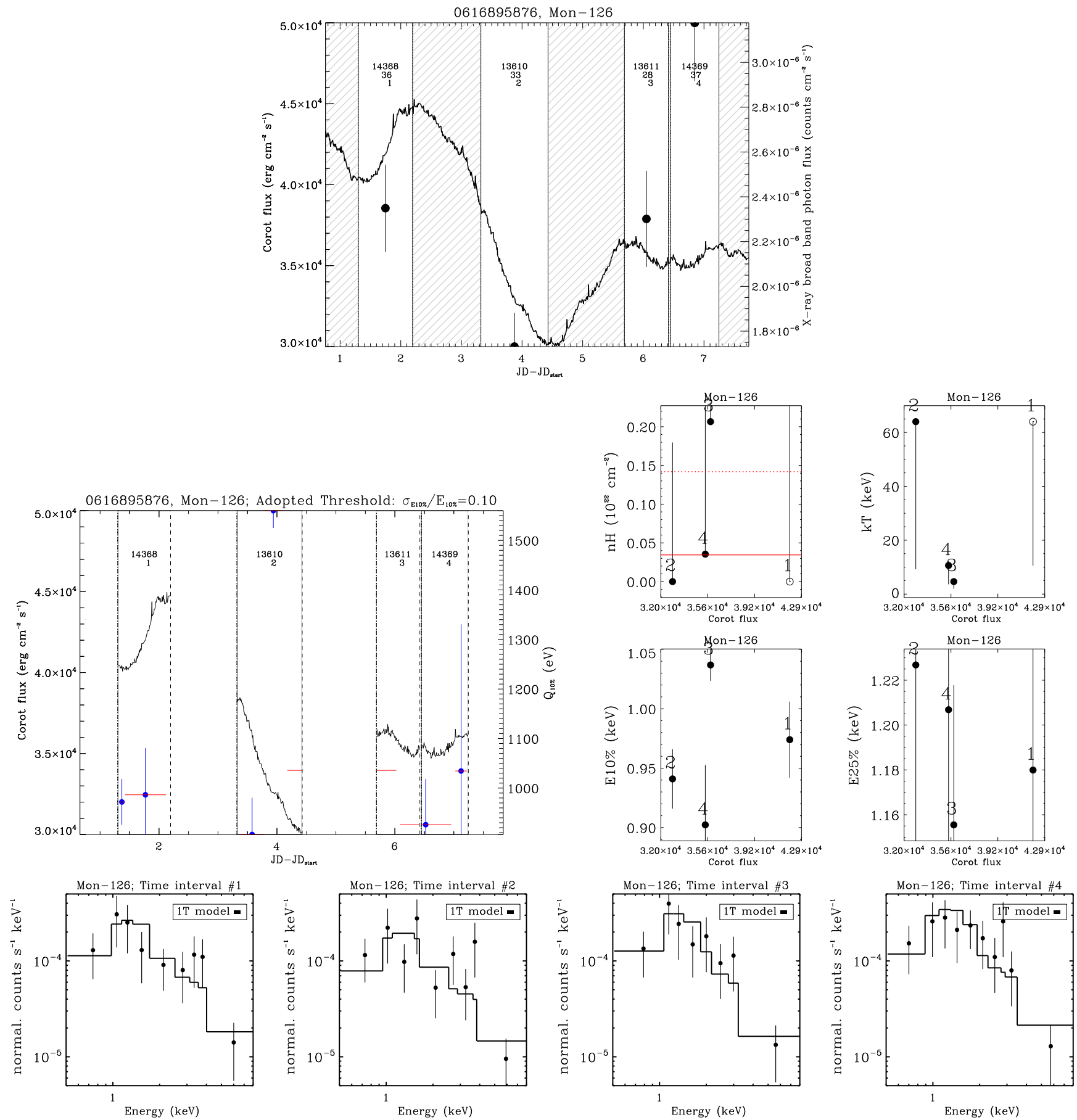

Fig. C.4. Variability and X-ray spectra of Mon-126, analyzed as a dipper. The CoRoT light curve shows dips without evidence of increasing $N_{\mathrm{H}}$. 

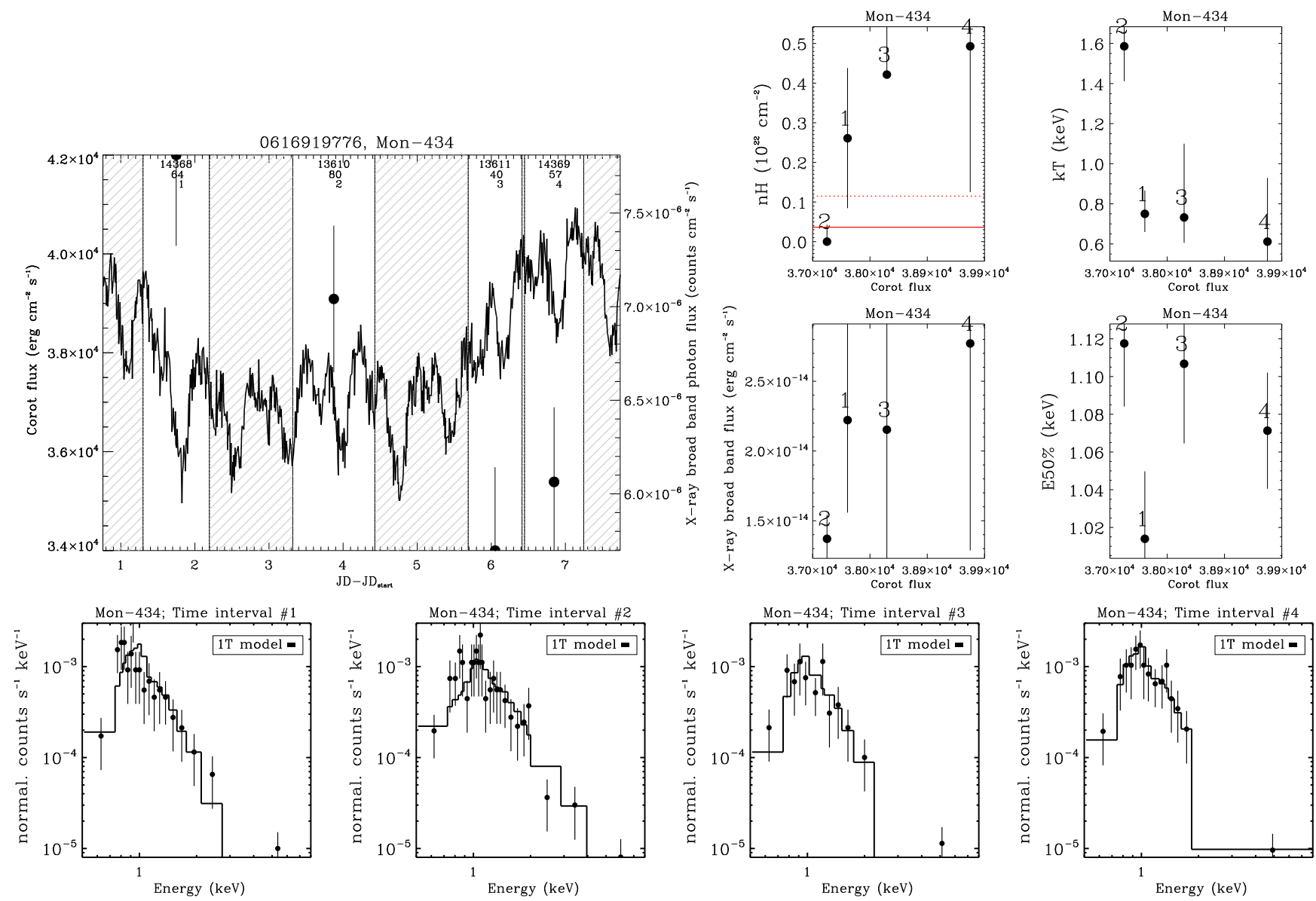

Fig. C.5. Variability and X-ray spectra of Mon-434. The CoRoT light curve is dominated by rotational modulation and likely pulsation, and it has been classified as a multi-periodic star by Cody et al. (2014). There may be evidence for correlated X-ray and CoRoT flux variability, but it is not significant. 
M. G. Guarcello et al.: CSI 2264: Simultaneous optical and X-ray variability in pre-main sequence stars
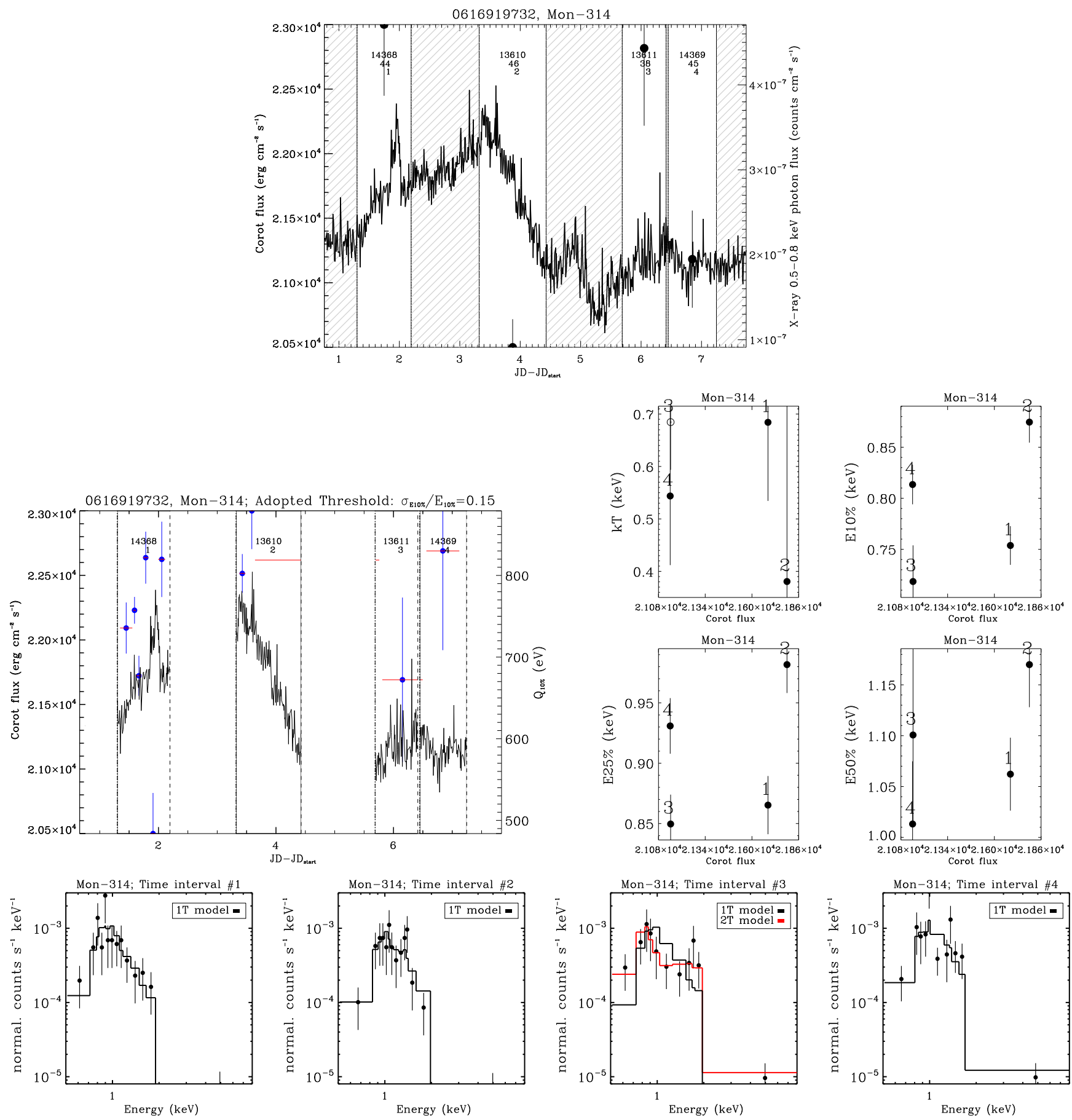

Fig. C.6. Variability and X-ray spectra of Mon-314, analyzed as a burster. The CoRoT light curve shows evident bursts and dips, but the poor $\mathrm{X}$-ray count rate does not allow us to analyze the simultaneous X-ray variability. During \#1, the X-ray spectrum becomes very soft during the optical burst, as shown by the variability of $E_{10 \%}$. The fit with $2 \mathrm{~T}$ thermal plasma model in \#3 is not well constrained due to the low X-ray count rate. 
A\&A 602, A10 (2017)
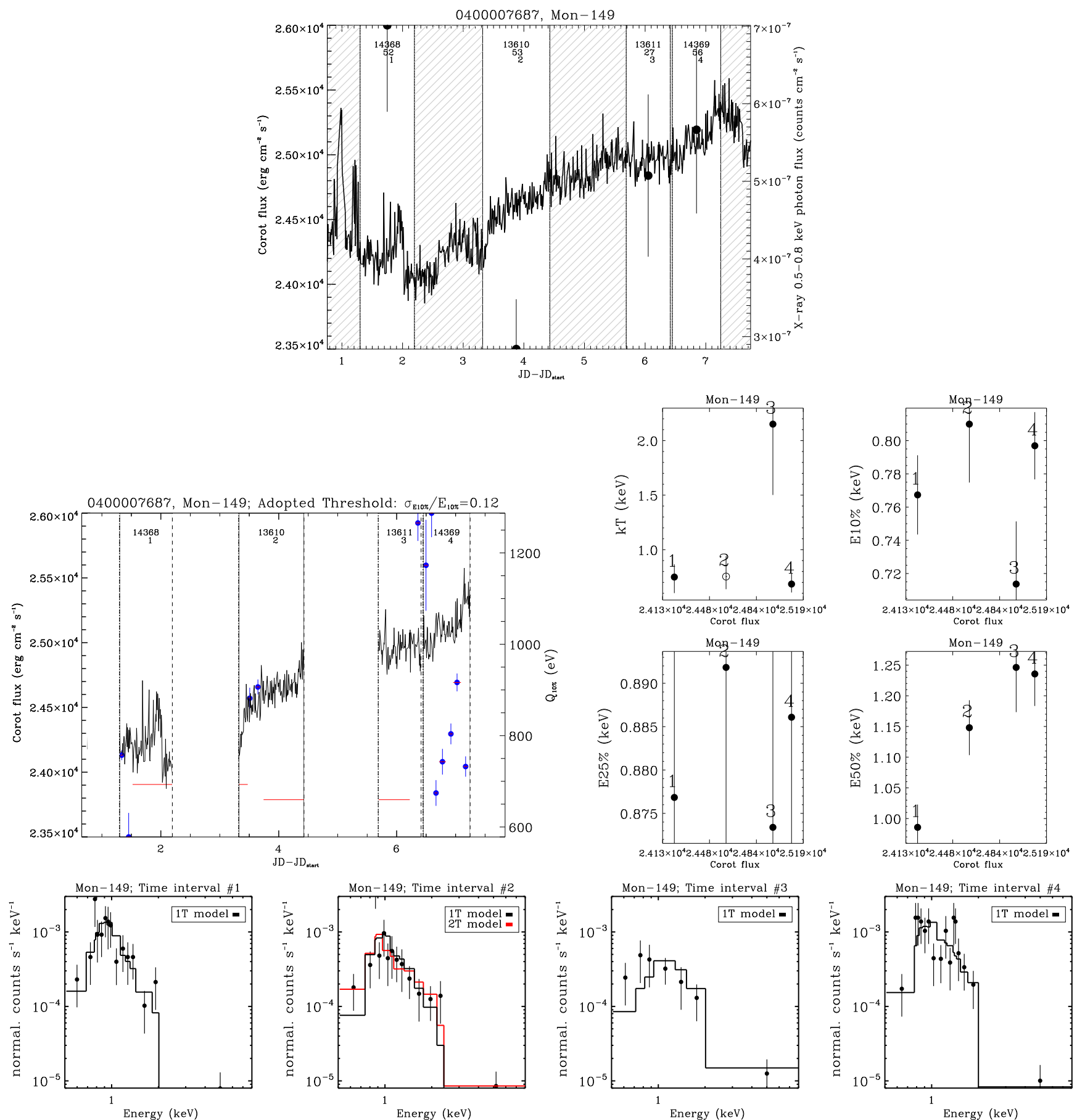

Fig. C.7. Variability and X-ray spectra of Mon-149, analyzed as a burster. The CoRoT light curve shows evident bursts and dips but few X-ray photons are detected. The variability of $E_{10 \%}$ shows interesting patterns, such as during \#4 when it rises until it declines significantly when a large burst occurs at the end of the interval. The X-ray spectrum observed during \#3 is soft, but it is fitted with 1T thermal model. It is not clear whether or not a soft X-ray spectral component is observed during \#2. 
M. G. Guarcello et al.: CSI 2264: Simultaneous optical and X-ray variability in pre-main sequence stars
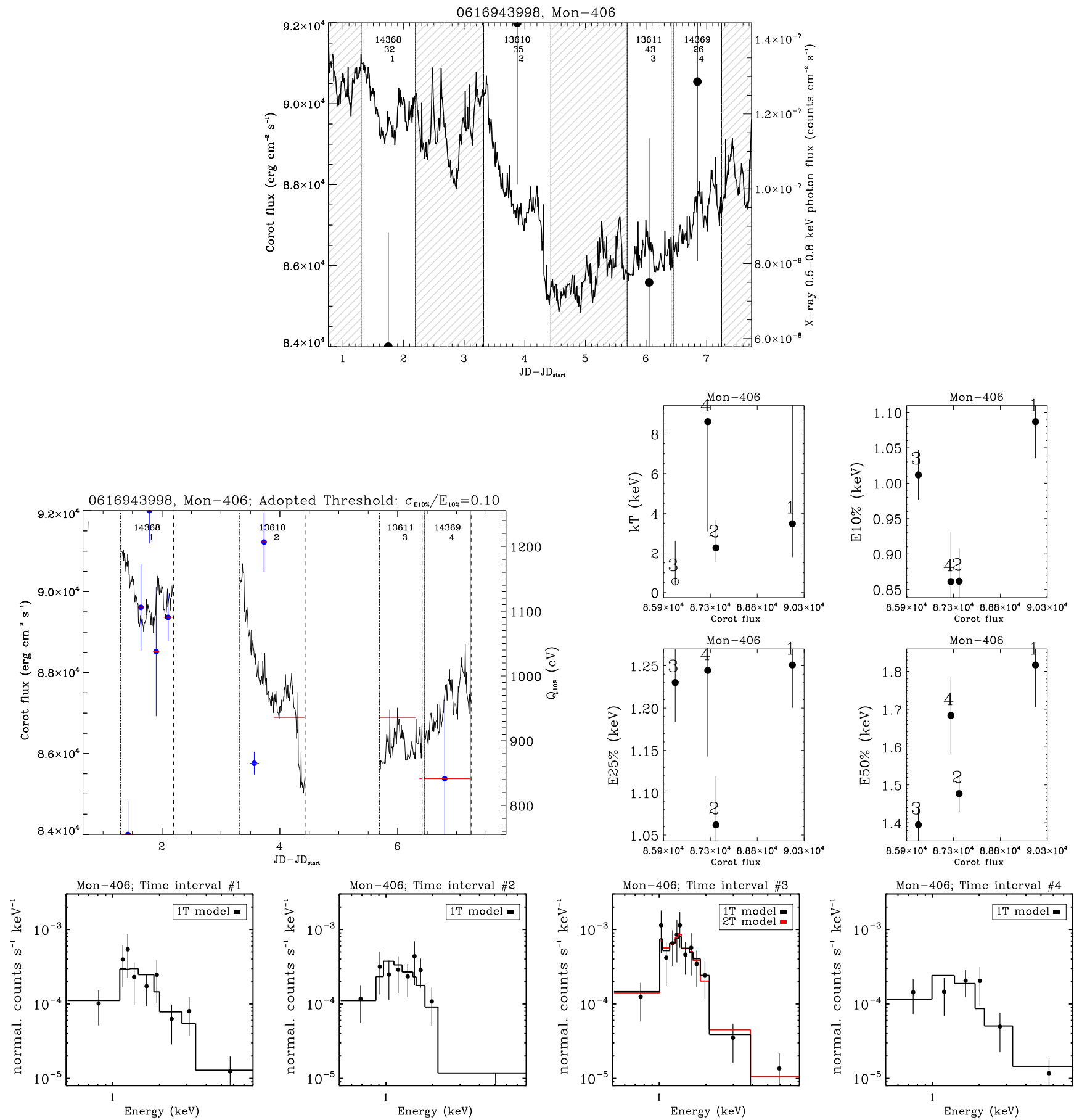

Fig. C.8. Variability of Mon-406, analyzed as a burster. The CoRoT light curve shows evident bursts and dips but few X-ray counts are detected. 
A\&A 602, A10 (2017)
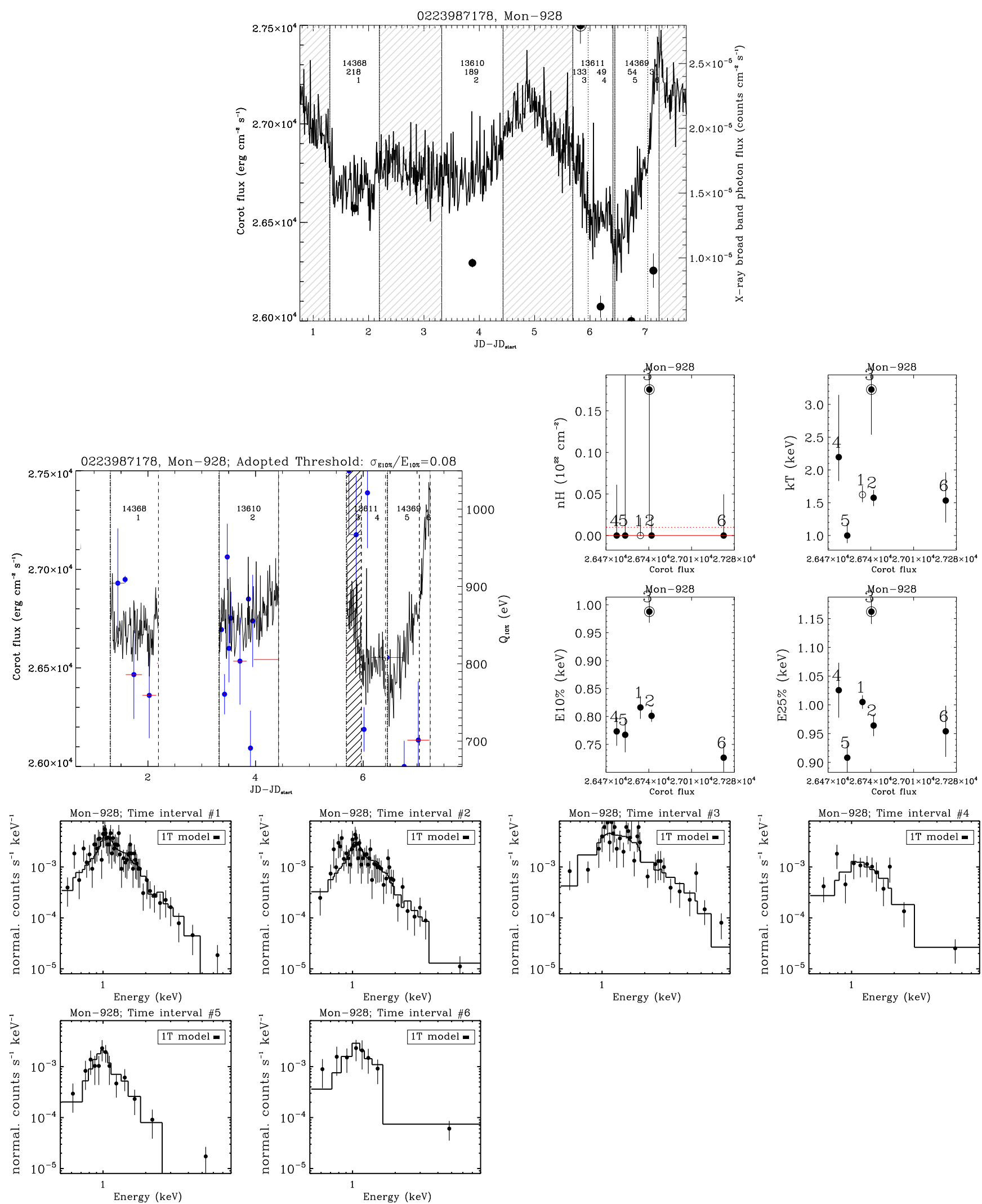

Fig. C.9. Variability and X-ray spectra of Mon-928 analyzed as a dipper. There is no evidence for increasing $N_{\mathrm{H}}$ during the optical dip (\#4 and \#5) and it may have soft X-ray emission during the burst (\#6), but few photons have been detected. 
M. G. Guarcello et al.: CSI 2264: Simultaneous optical and X-ray variability in pre-main sequence stars
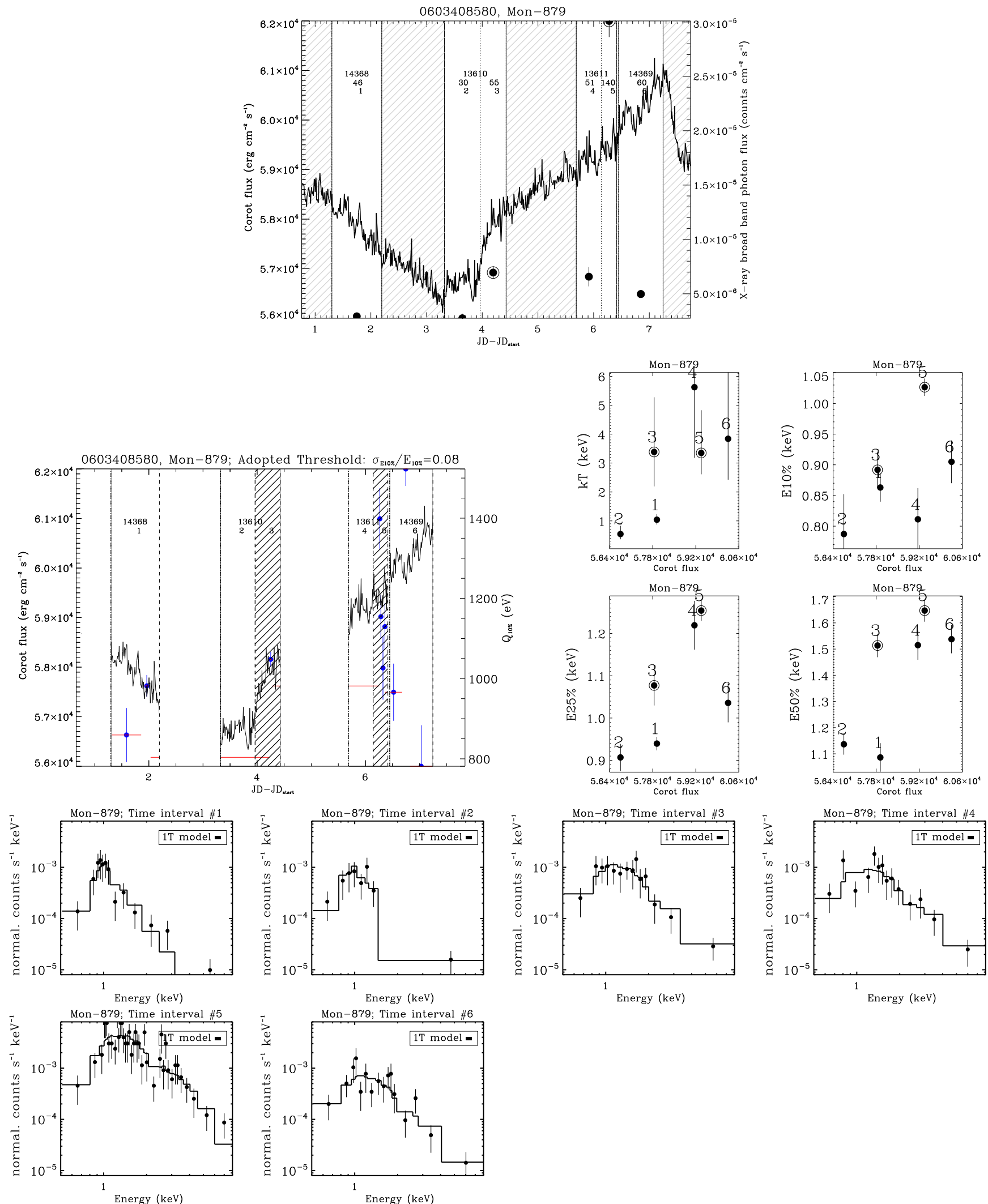

Fig. C.10. Variability and X-ray spectra of Mon-879, analyzed as a burster. There is no evidence for soft X-ray emission during the optical bursts, and the CoRoT mask may have been contaminated by a nearby bright optical source. 
A\&A 602, A10 (2017)
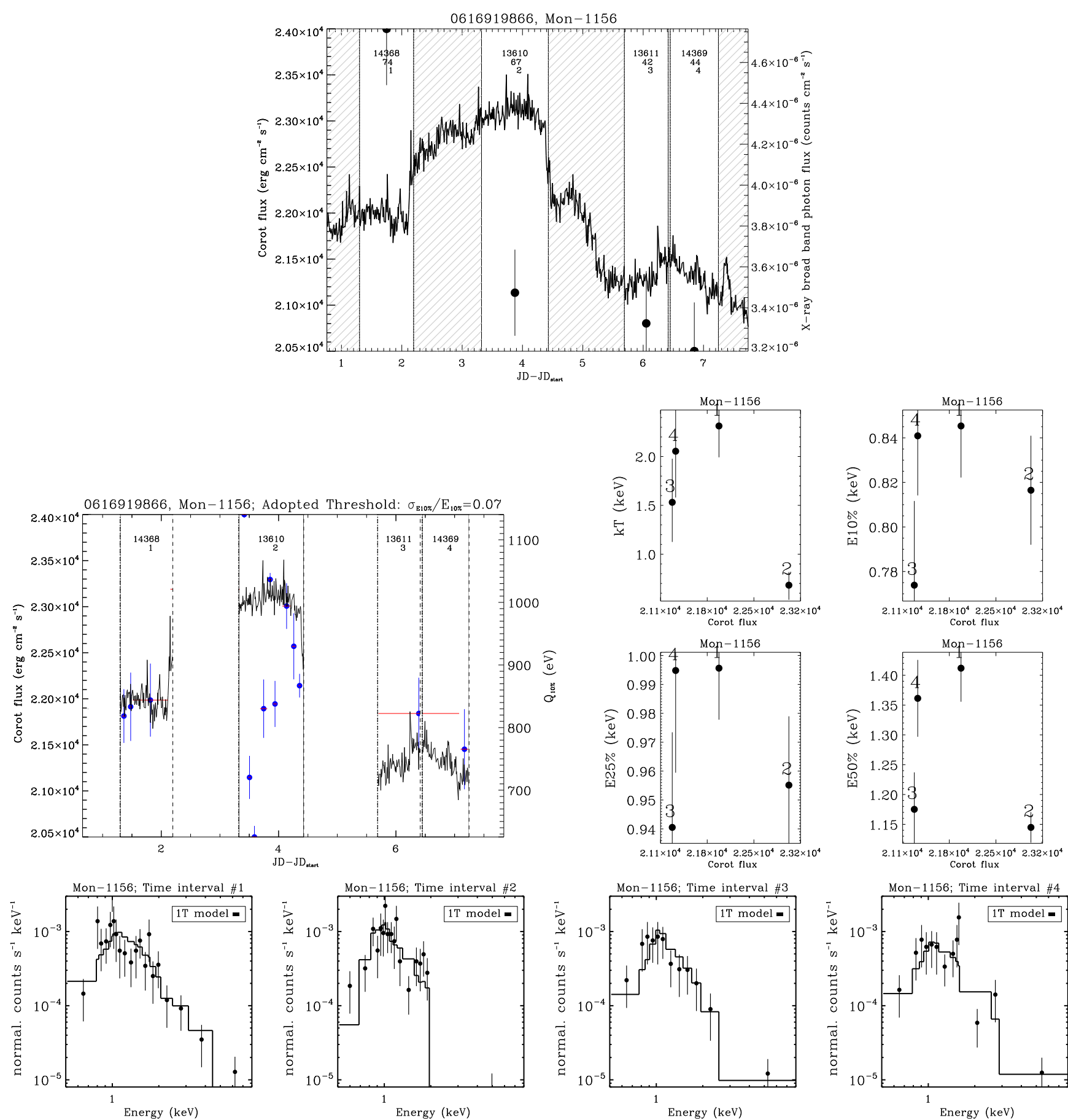

Fig. C.11. Variability and X-ray spectra of Mon-1156, analyzed as a burster. No significant X-ray variability is observed. 
M. G. Guarcello et al.: CSI 2264: Simultaneous optical and X-ray variability in pre-main sequence stars
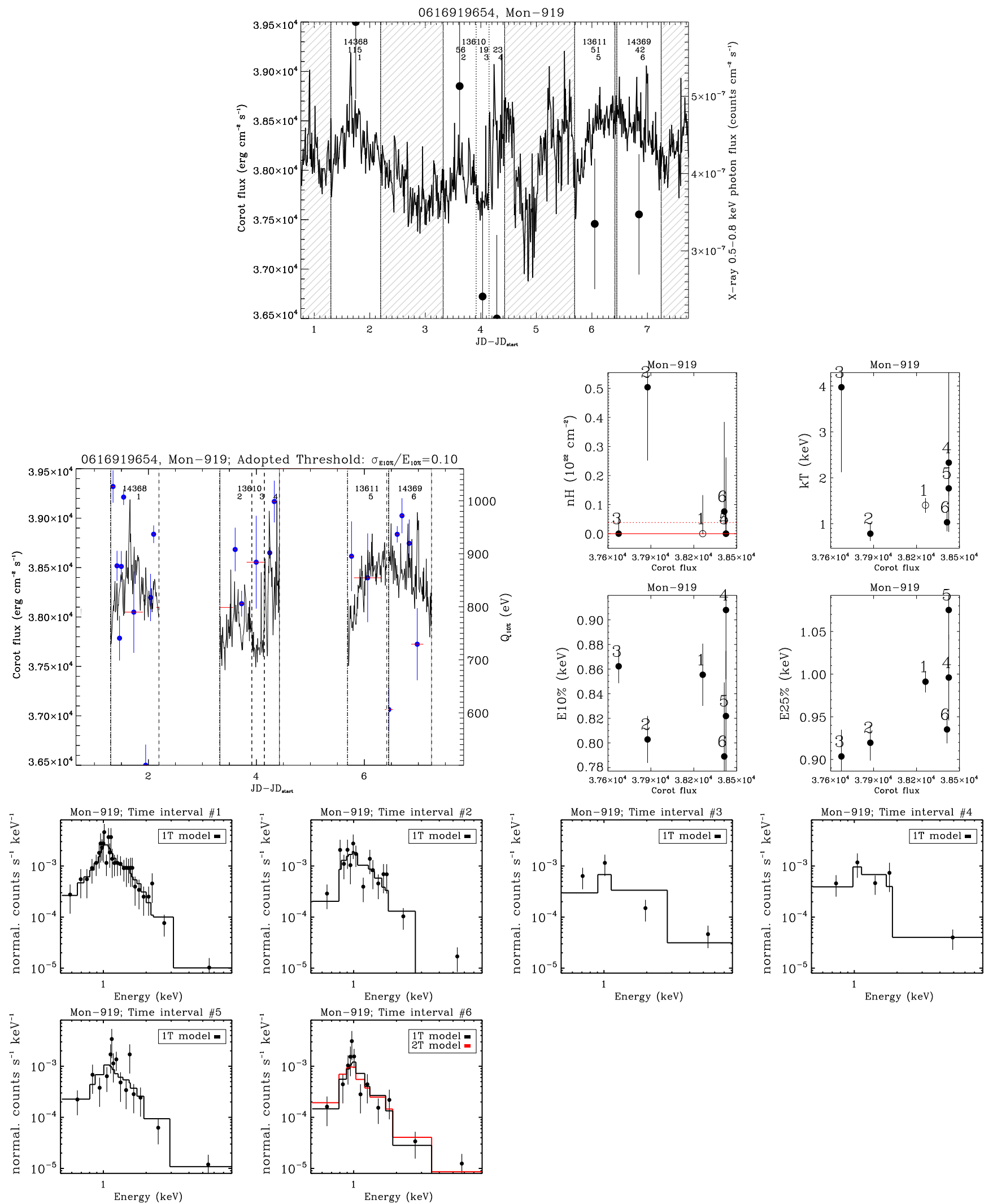

Fig. C.12. Variability and X-ray spectra of Mon-919, analyzed both as a dipper and burster, with no significant correlation between the optical and $\mathrm{X}$-ray variability. 
A\&A 602, A10 (2017)
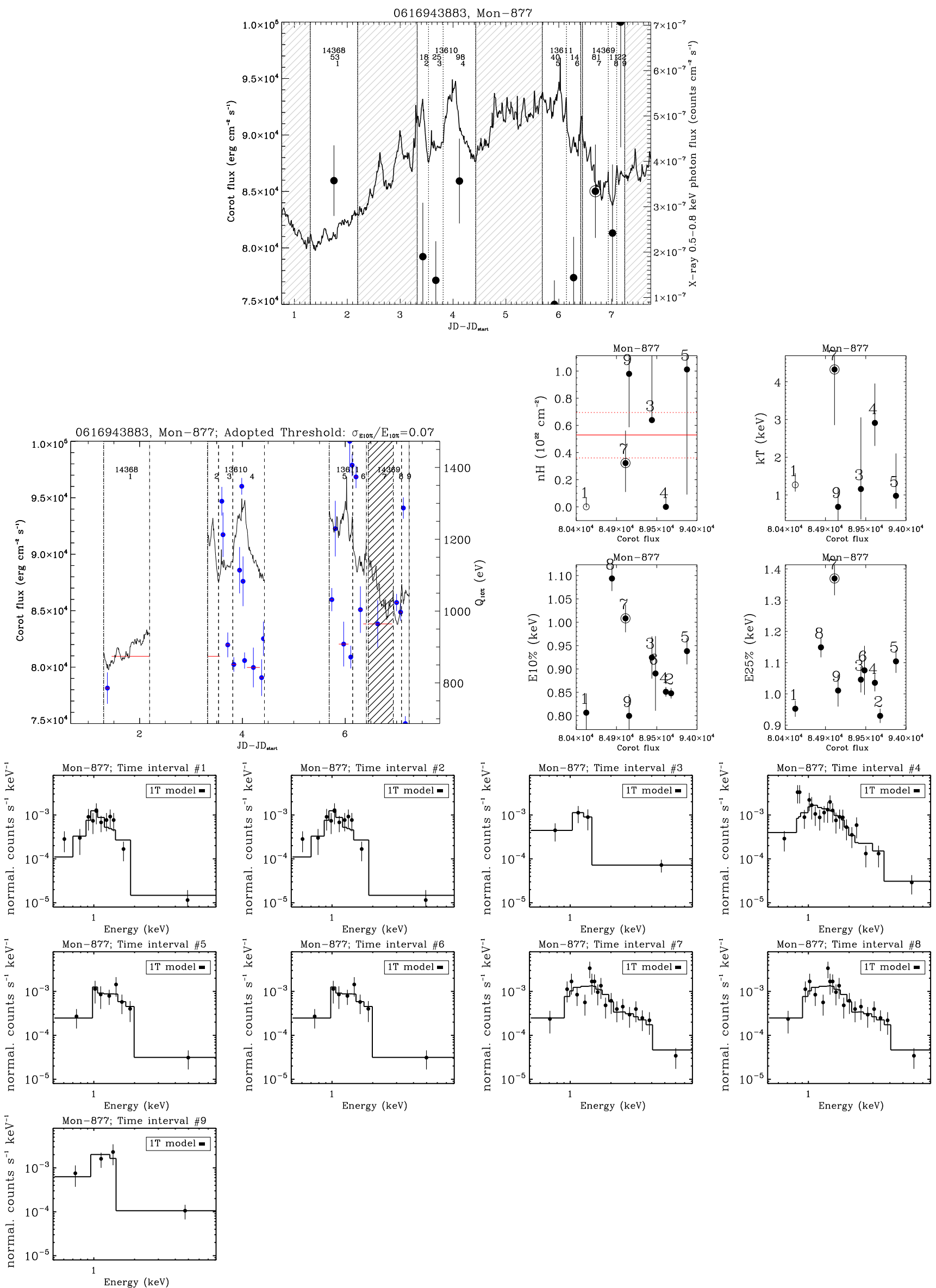

Fig. C.13. Variability and X-ray spectra of Mon-877, analyzed both as a dipper and burster, with no significant correlation between the optical and $\mathrm{X}$-ray variability. 
M. G. Guarcello et al.: CSI 2264: Simultaneous optical and X-ray variability in pre-main sequence stars
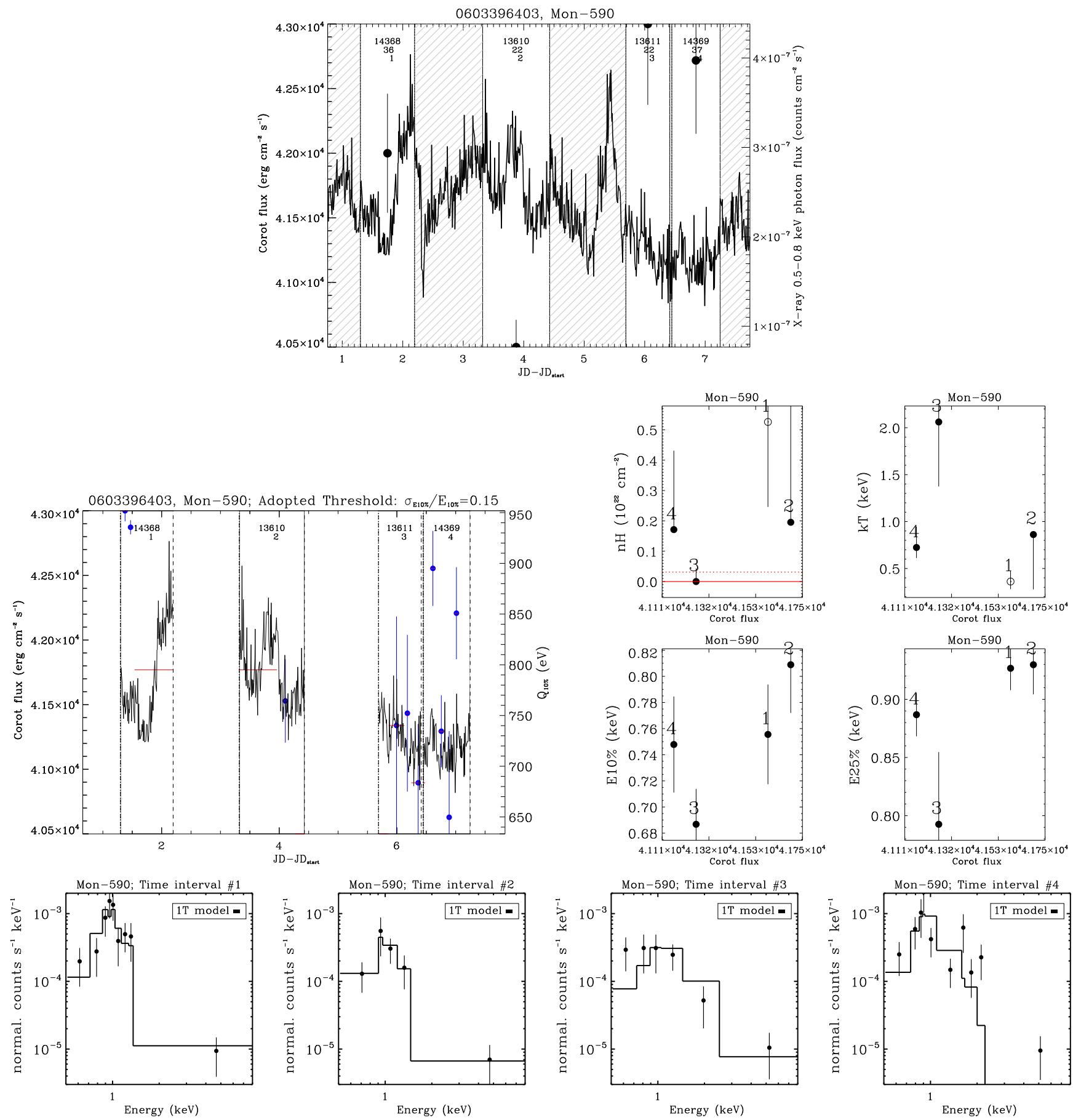

Fig. C.14. Variability and X-ray spectra of Mon-590, analyzed both as a dipper and burster, but with few X-ray photons detected. 

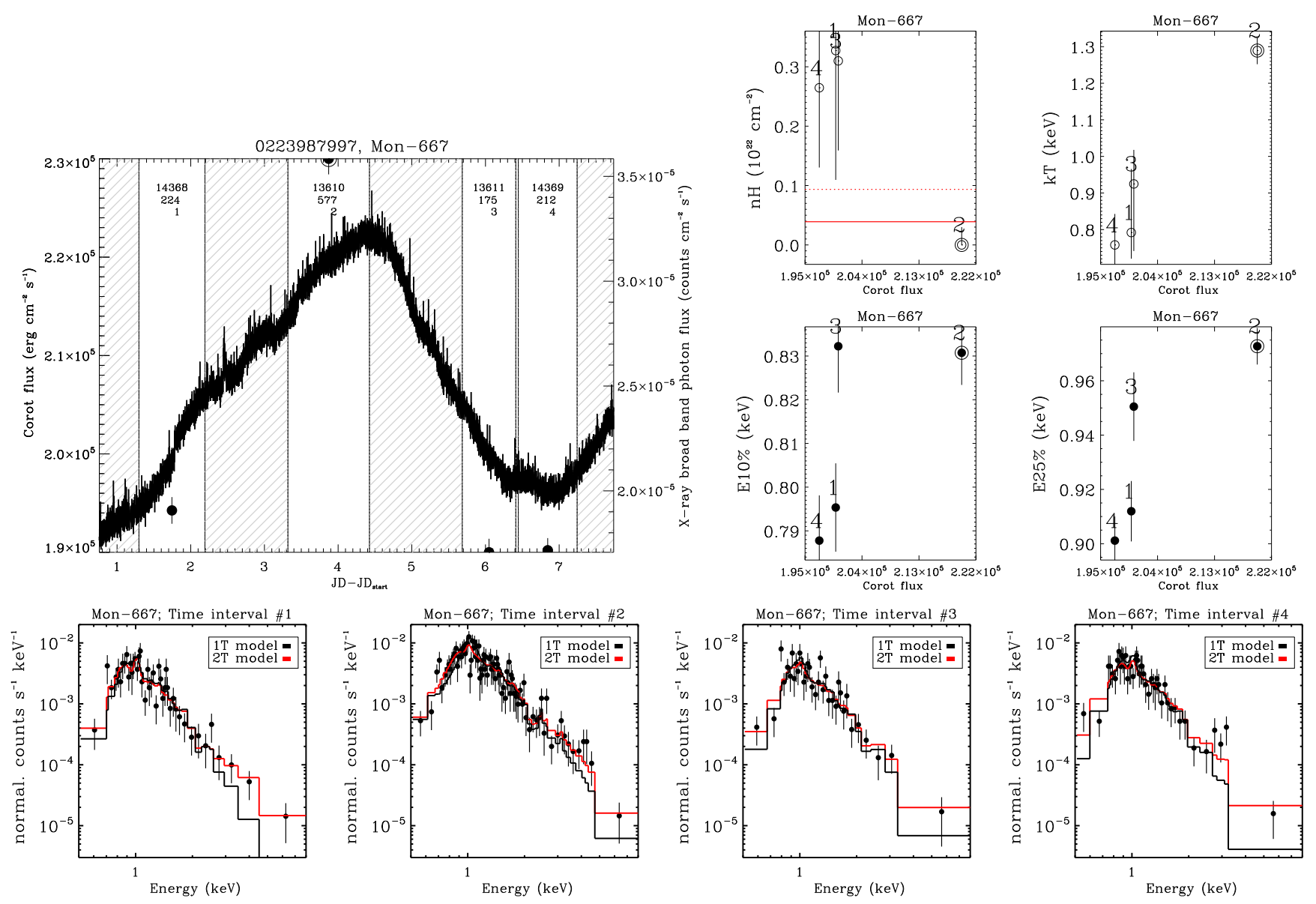

Fig. C.15. Variability and X-ray spectra of Mon-667. During the Chandra frames, Mon-667 mimics a quasi-periodic optical variability, but the CoRoT light curve shows evident dips after the Chandra observations. We analyzed it as a dipper, but the fit of the X-ray spectrum with 1T and $2 \mathrm{~T}$ thermal plasma models are never well constrained. 
M. G. Guarcello et al.: CSI 2264: Simultaneous optical and X-ray variability in pre-main sequence stars
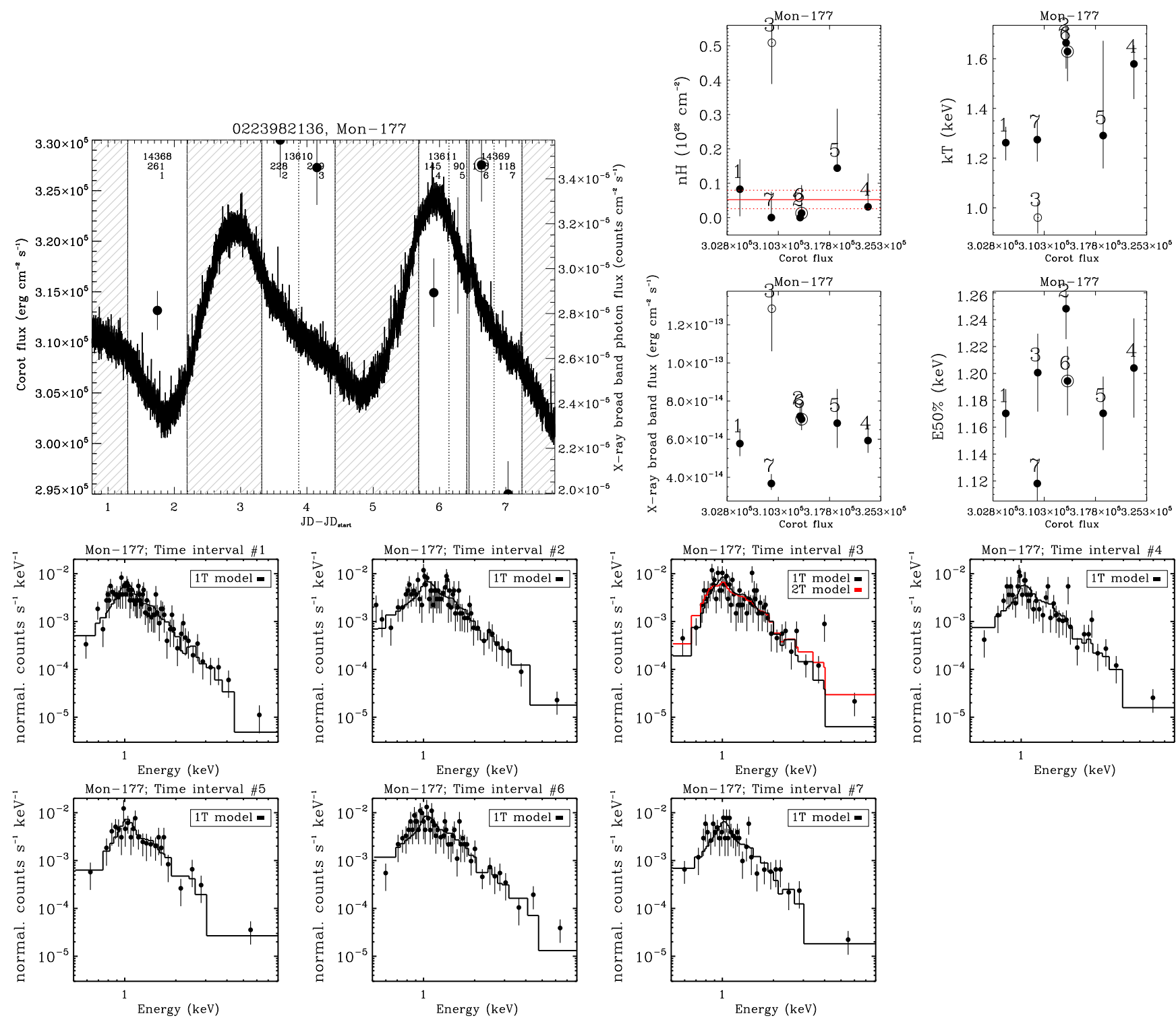

Fig. C.16. Variability and X-ray spectra of Mon-177, showing periodic optical variability but no interesting X-ray variability. 

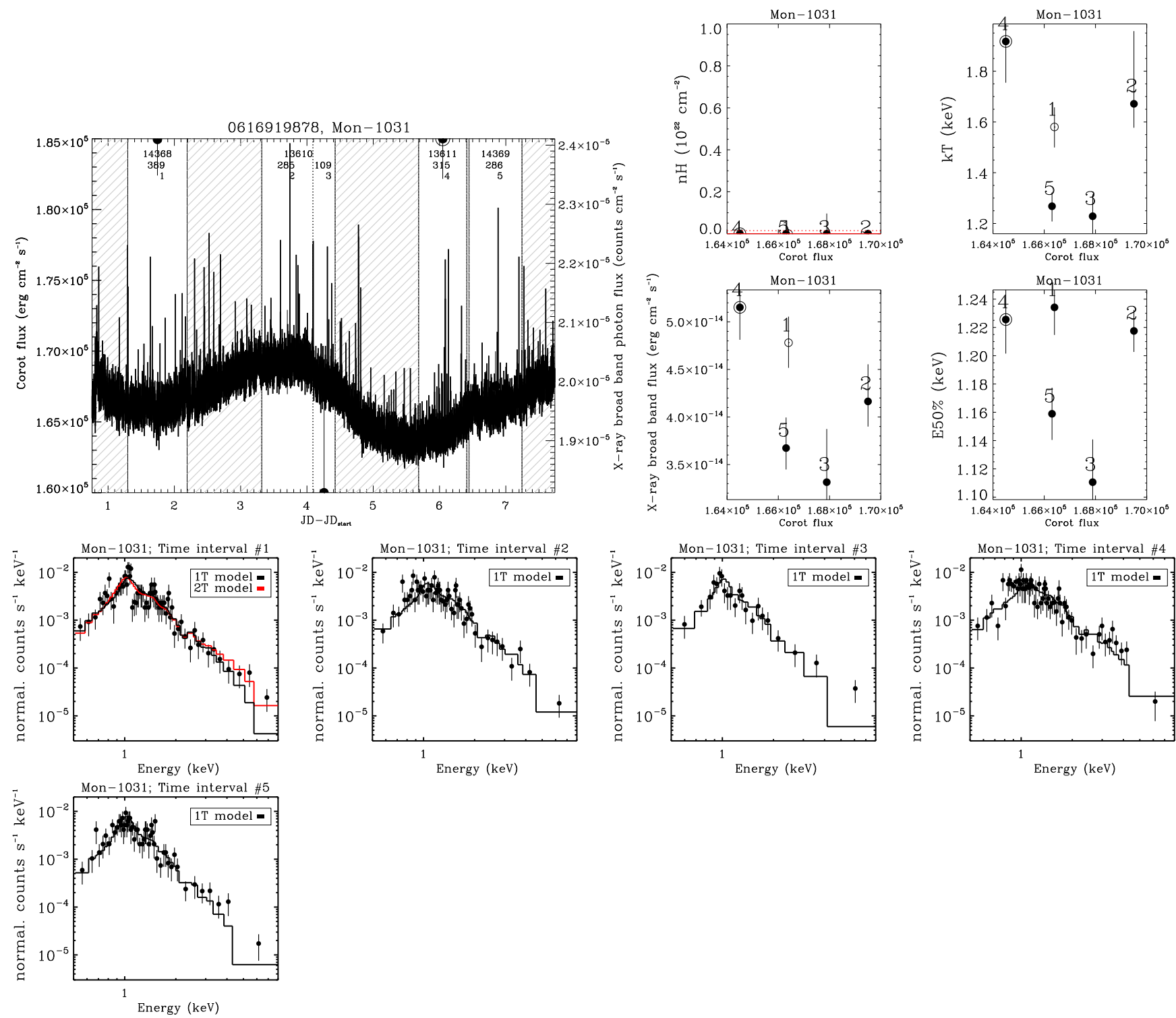

Fig. C.17. Variability and X-ray spectra of Mon-1031, showing periodic optical variability but no interesting X-ray variability. 
M. G. Guarcello et al.: CSI 2264: Simultaneous optical and X-ray variability in pre-main sequence stars
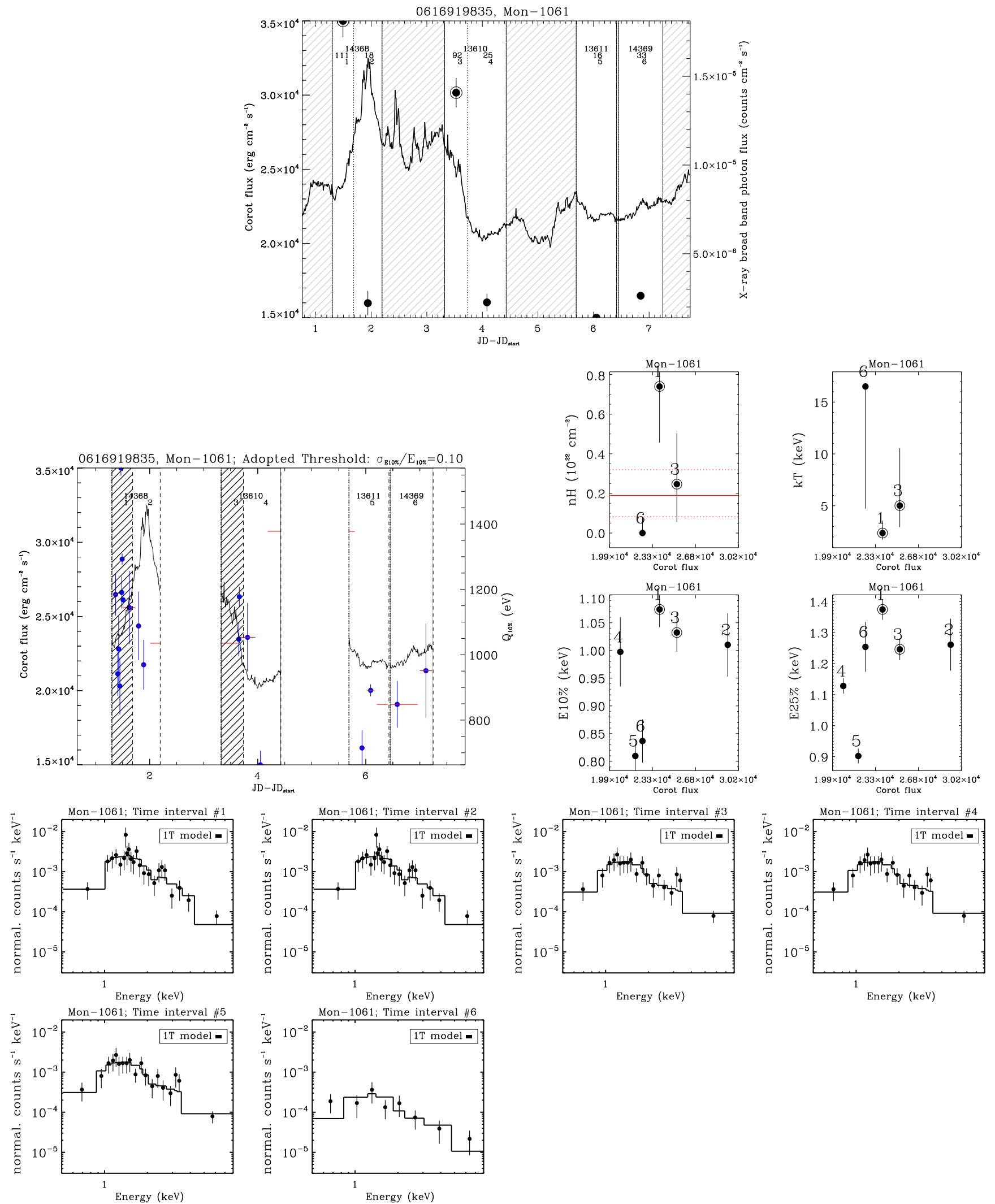

Fig. C.18. Variability and X-ray spectra of Mon-1061, which has not been analyzed since very few X-ray photons are detected during the relevant optical features. 
A\&A 602, A10 (2017)
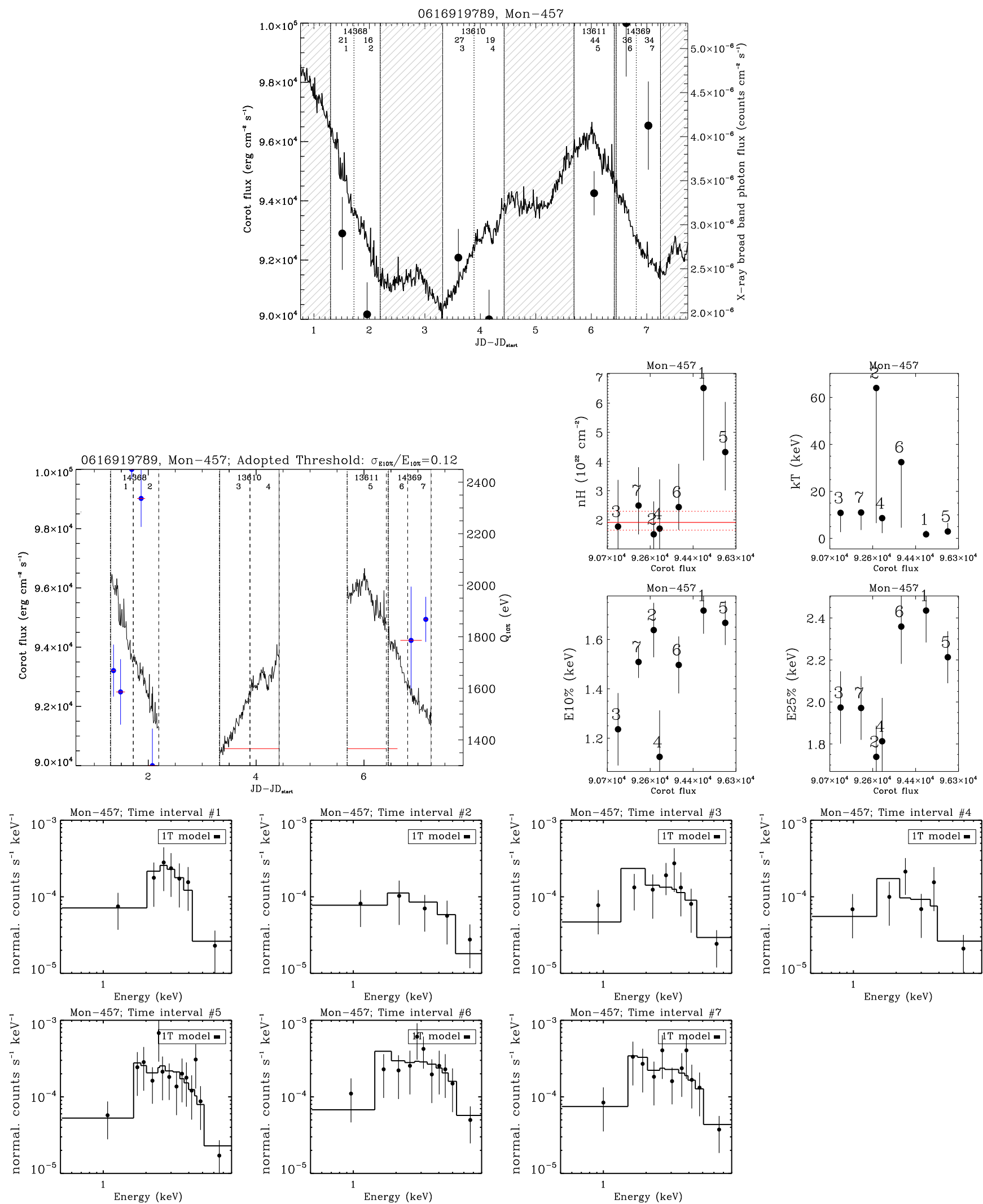

Fig. C.19. Variability and X-ray spectra of Mon-457, which has not been analyzed since very few X-ray photons are detected during the relevant optical features. 
M. G. Guarcello et al.: CSI 2264: Simultaneous optical and X-ray variability in pre-main sequence stars
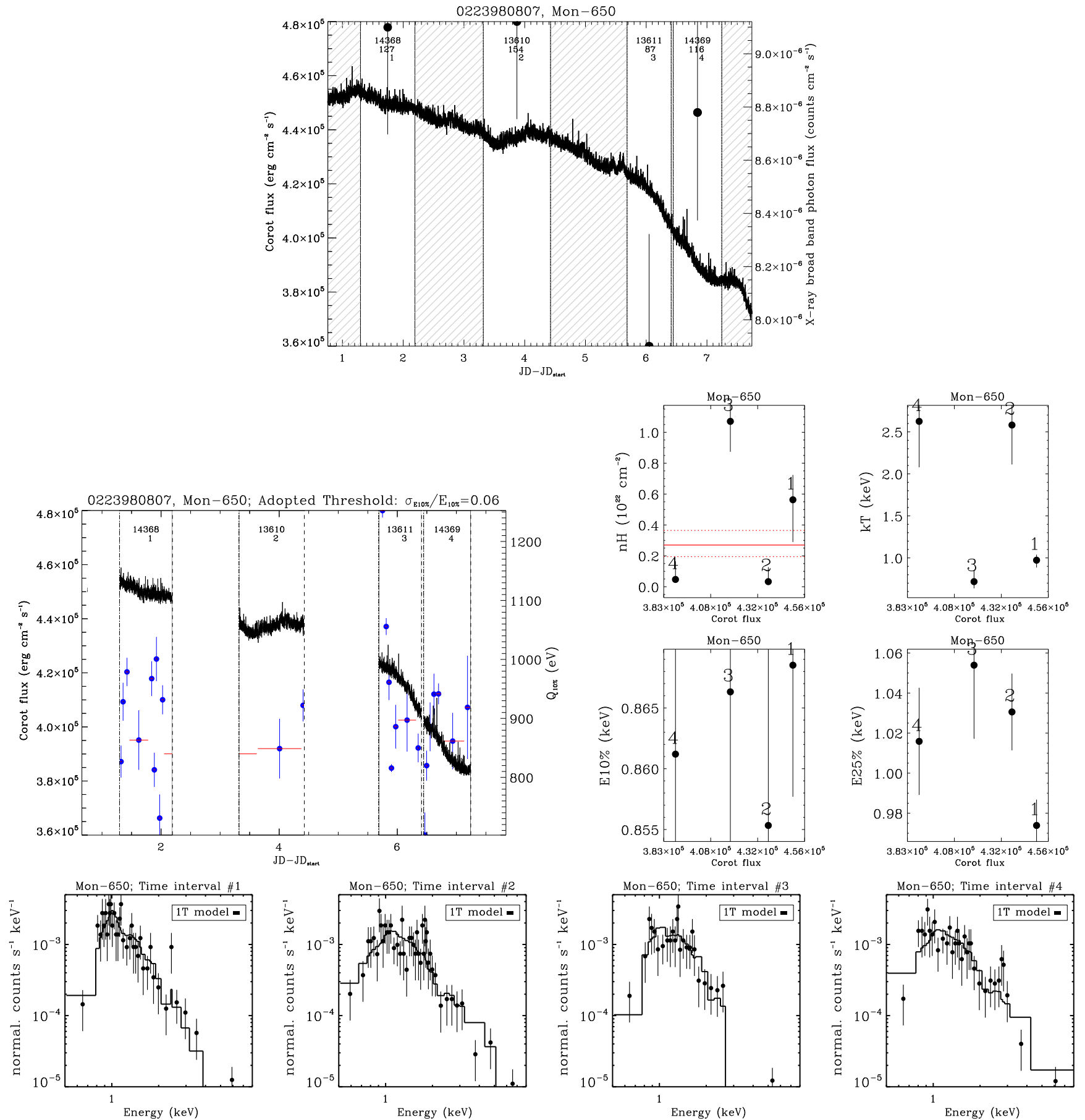

Fig. C.20. Variability and X-ray spectra of Mon-650, whose CoRoT light curve is dominated by large dips not occurring during the Chandra observations. 
A\&A 602, A10 (2017)
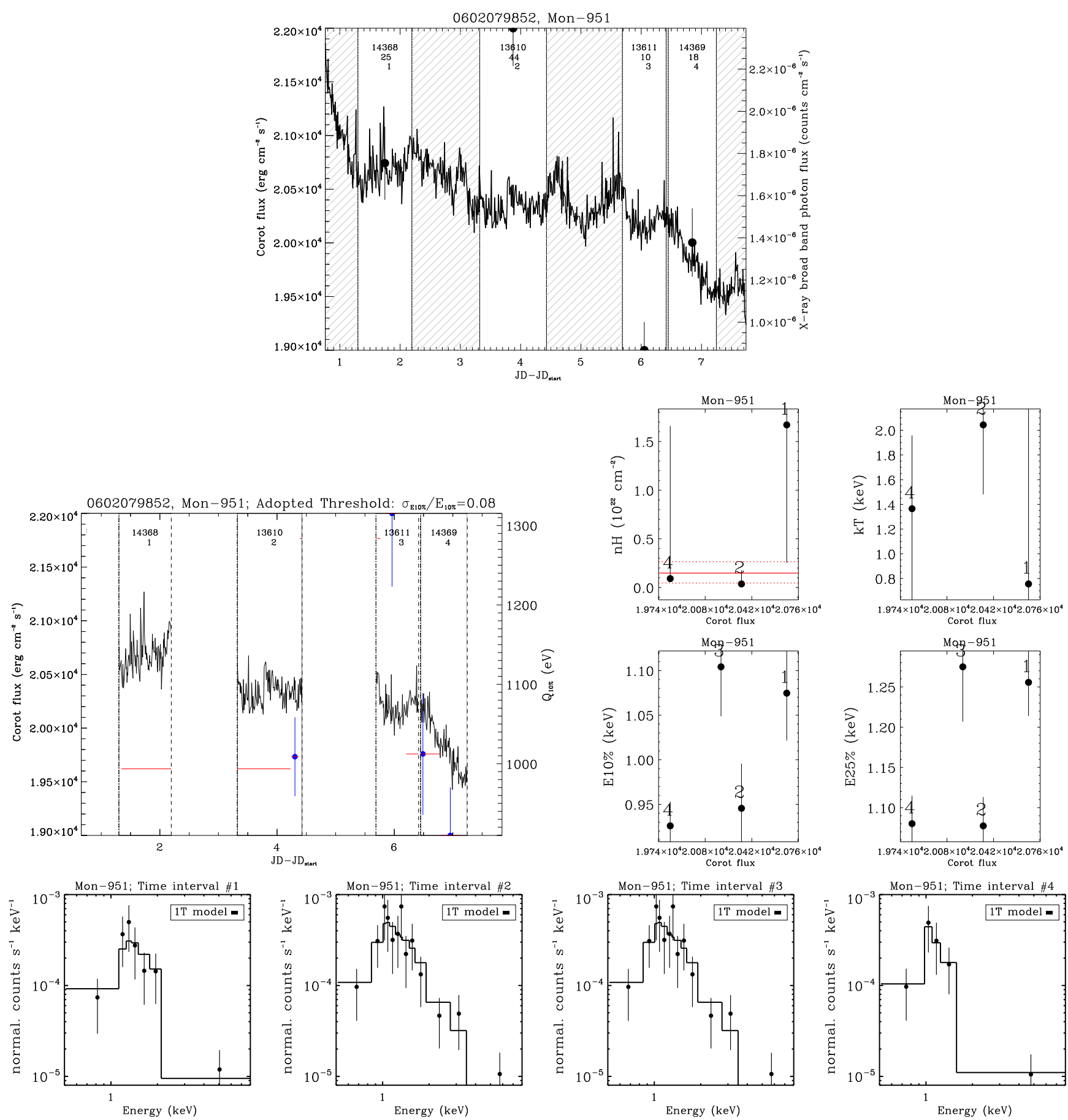

Fig. C.21. Variability and X-ray spectra of Mon-951, with no interesting variability of the X-ray properties observed and few photons detected. 
M. G. Guarcello et al.: CSI 2264: Simultaneous optical and X-ray variability in pre-main sequence stars
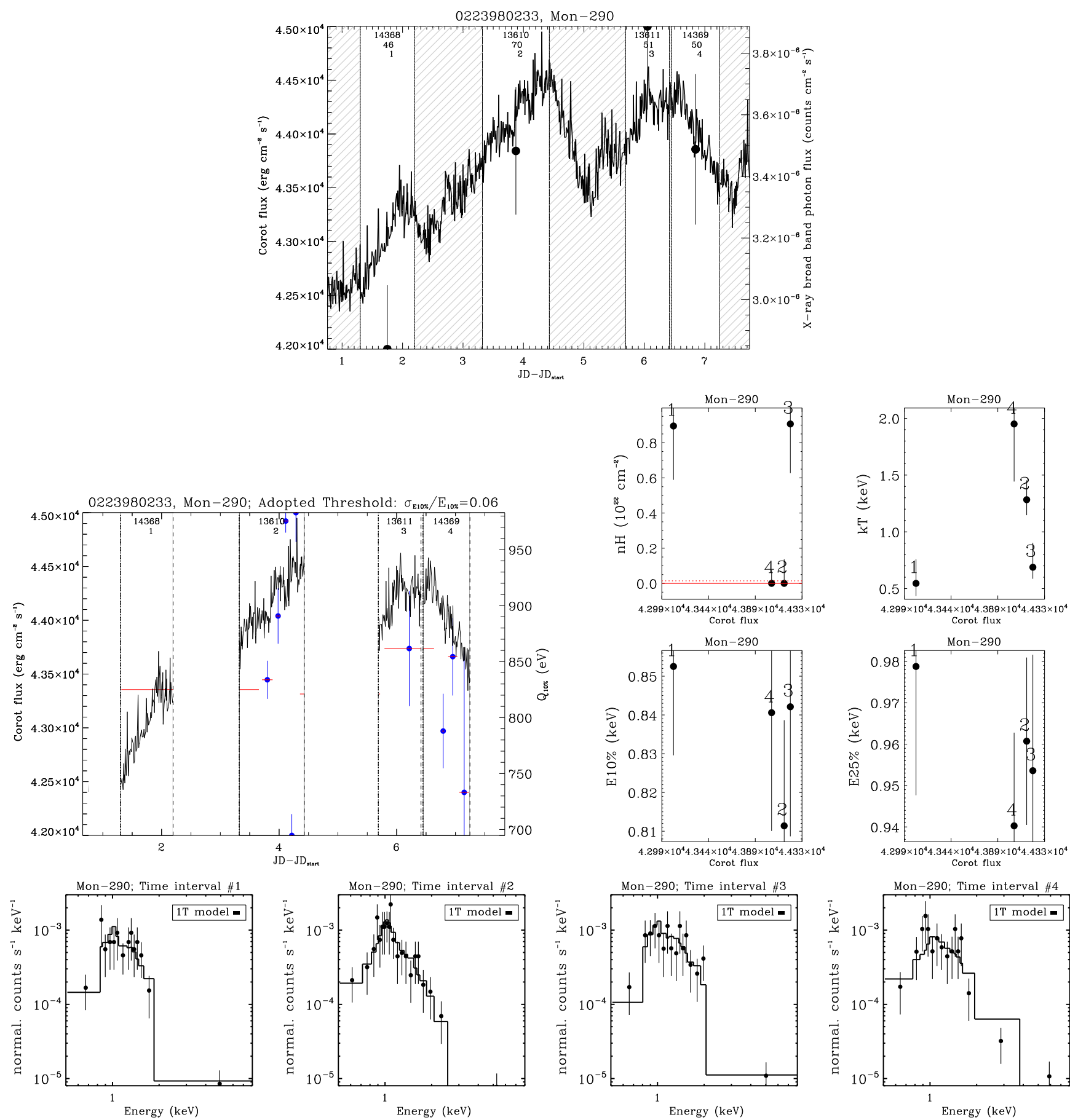

Fig. C.22. Variability and X-ray spectra of Mon-290, analyzed as a burster, but with no interesting variability of the X-ray properties observed. 
A\&A 602, A10 (2017)
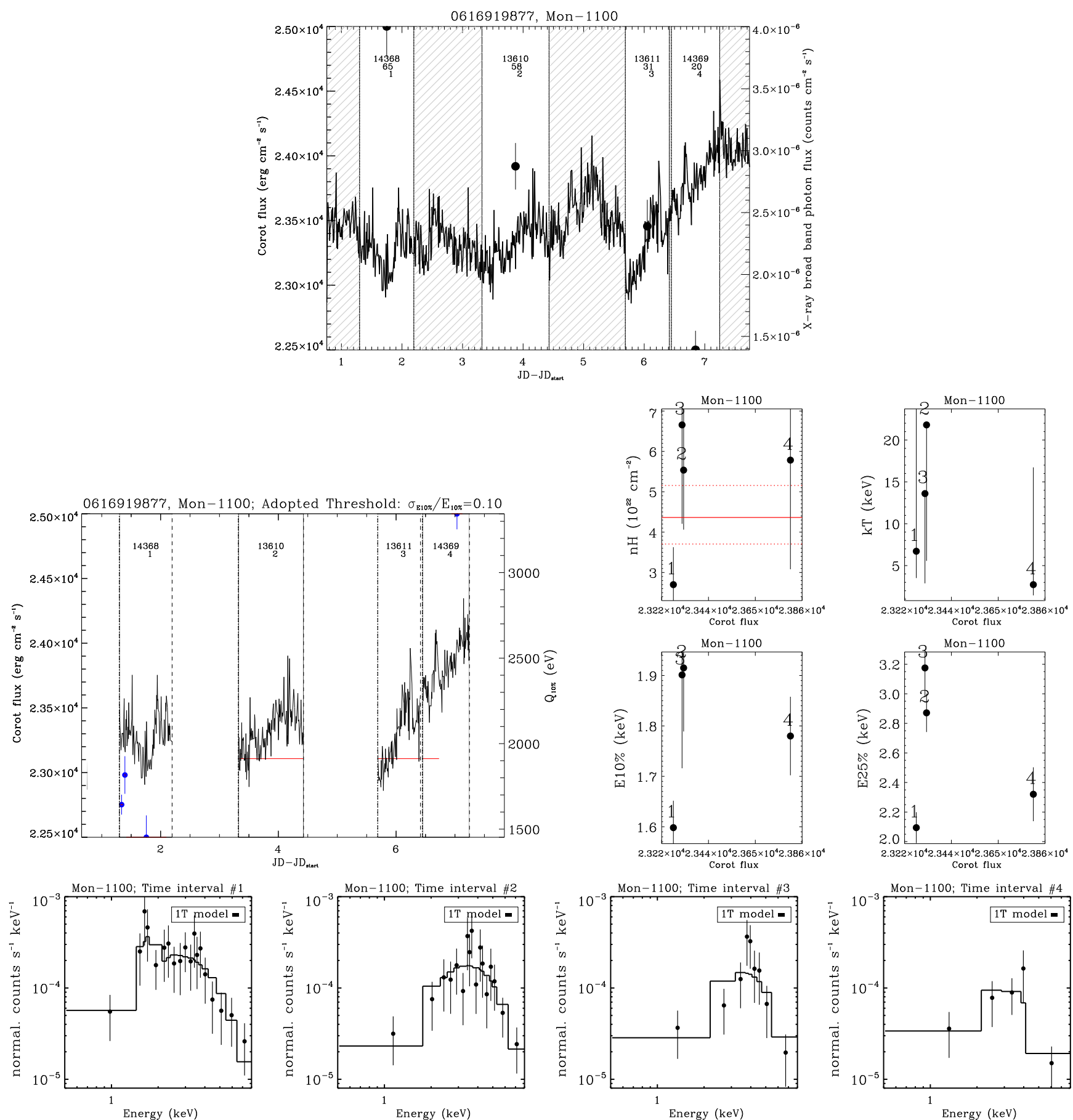

Fig. C.23. Variability and X-ray spectra of Mon-1100, with no interesting variability of the X-ray properties observed. 
M. G. Guarcello et al.: CSI 2264: Simultaneous optical and X-ray variability in pre-main sequence stars
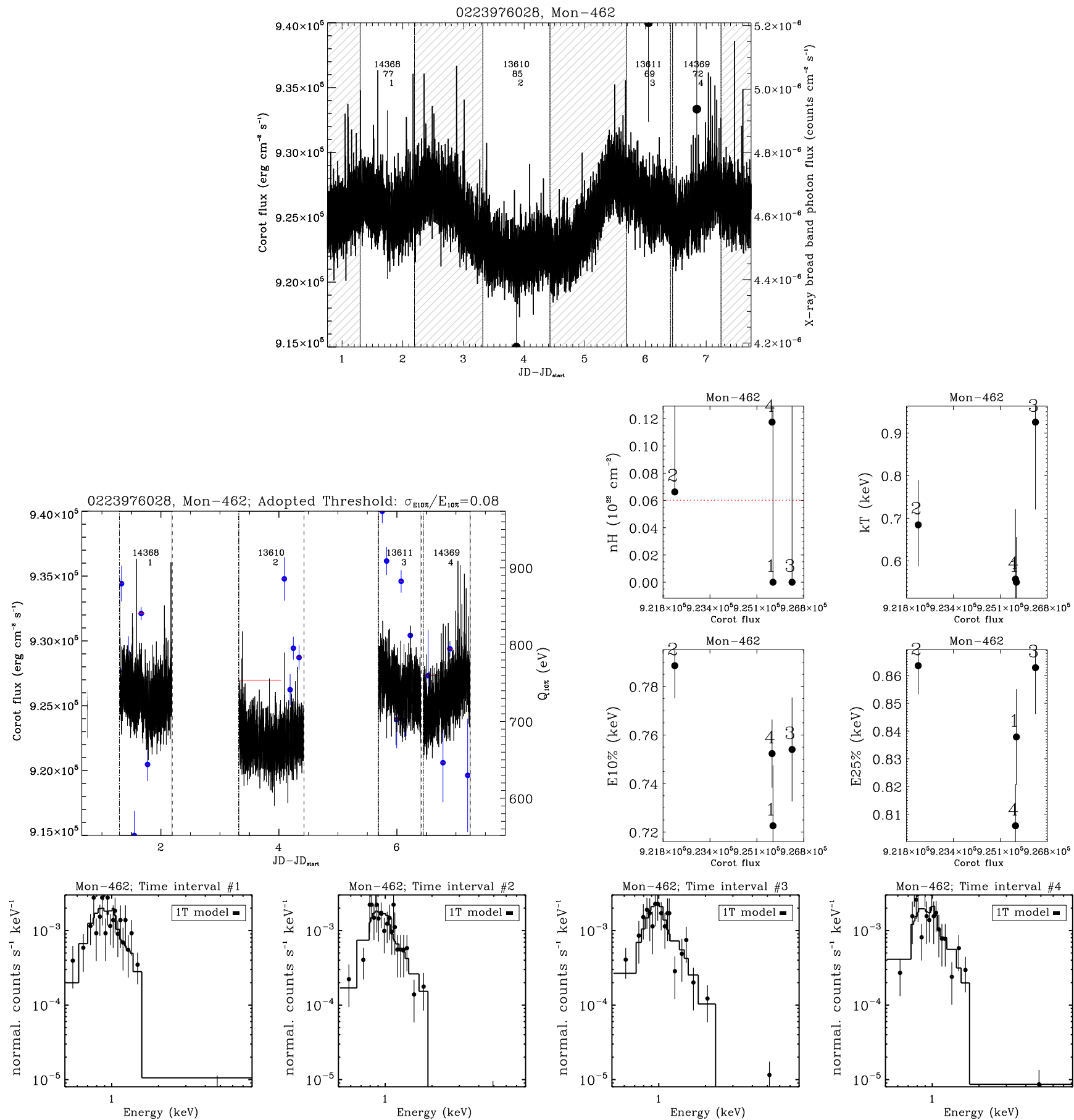

Fig. C.24. Variability and X-ray spectra of Mon-462, analyzed as a dipper, with a large optical dip observed, which however does not correspond to a significant increase of $N_{\mathrm{H}}$. 
A\&A 602, A10 (2017)
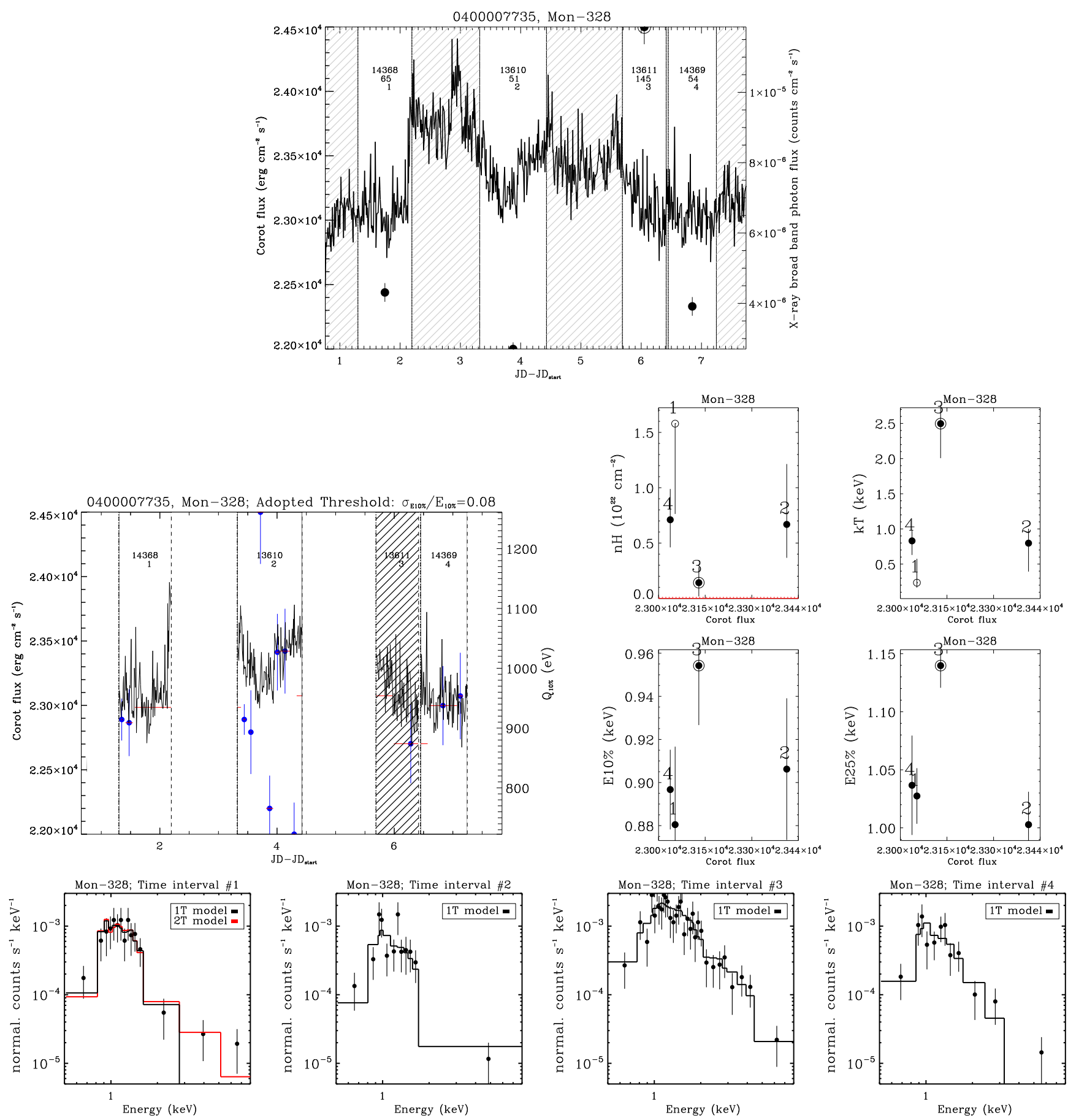

Fig. C.25. Variability and X-ray spectra of Mon-328, with no evident optical features observed during the Chandra frames. 
M. G. Guarcello et al.: CSI 2264: Simultaneous optical and X-ray variability in pre-main sequence stars
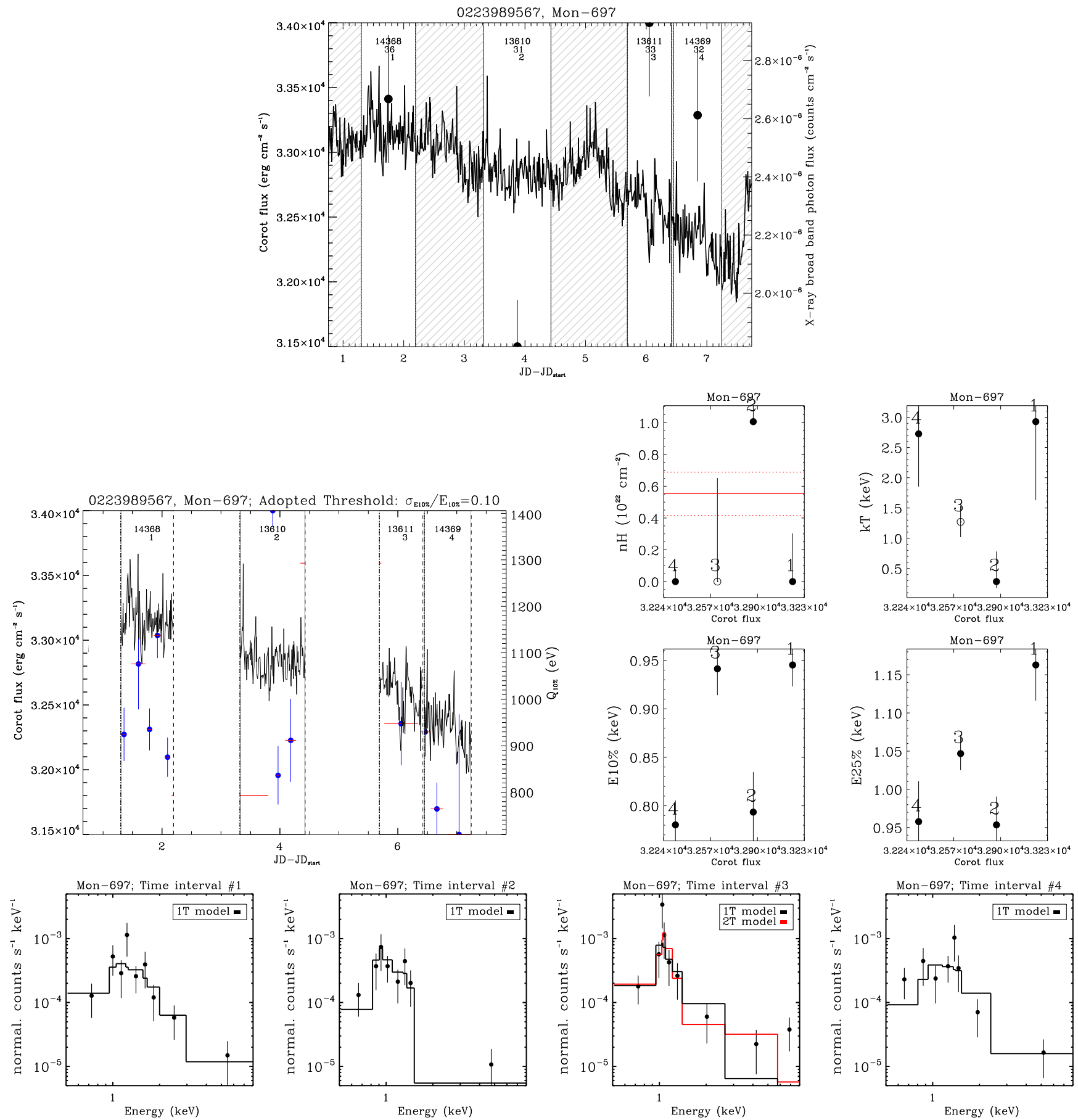

Fig. C.26. Variability and X-ray spectra of Mon-697, with no evident optical features observed during the Chandra frames and few X-ray photons detected. 
A\&A 602, A10 (2017)
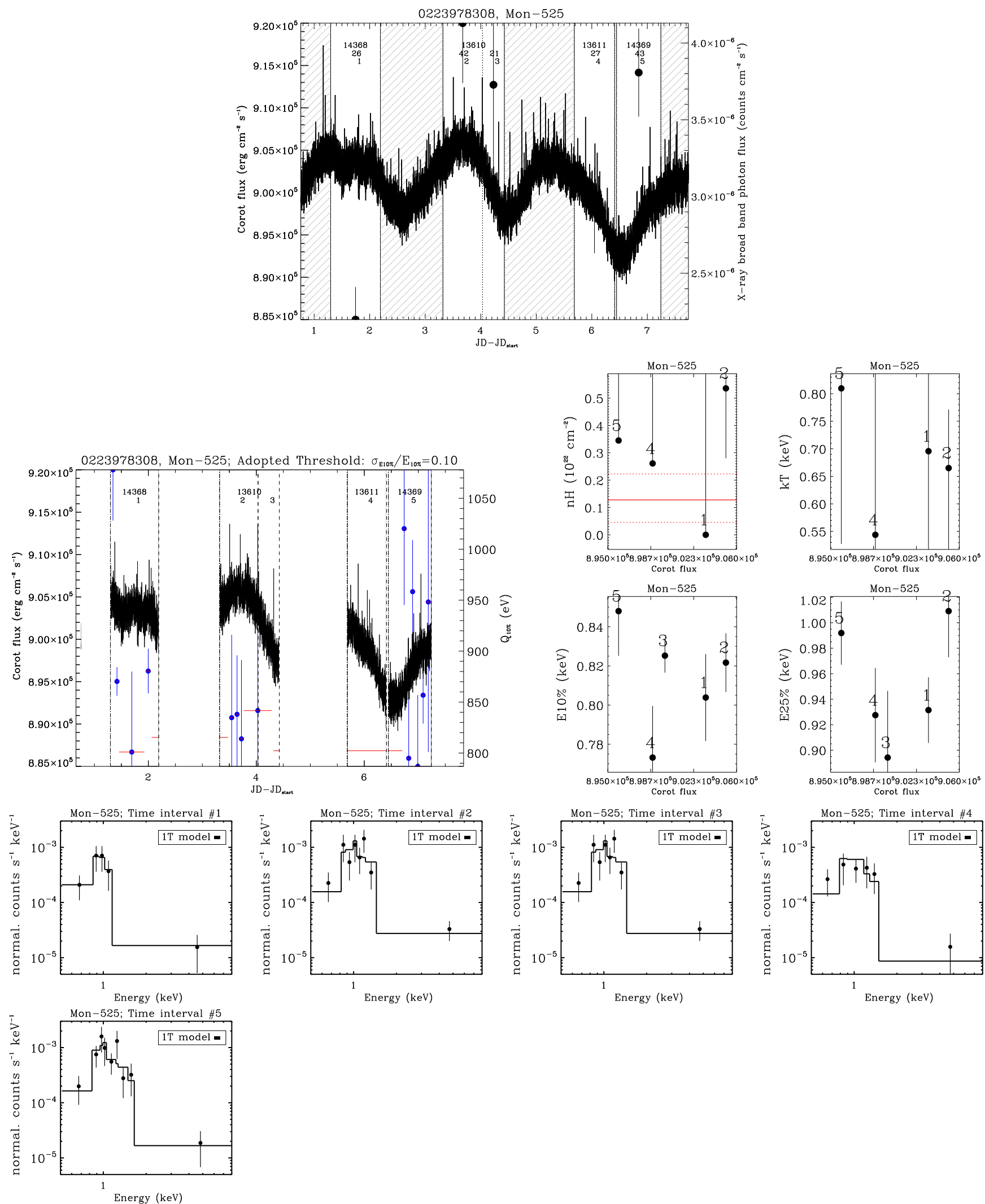

Fig. C.27. Variability and X-ray spectra of Mon-525, analyzed as a dipper. The CoRoT light curve shows dips that do not correspond to significant variations of $N_{\mathrm{H}}$. 
M. G. Guarcello et al.: CSI 2264: Simultaneous optical and X-ray variability in pre-main sequence stars
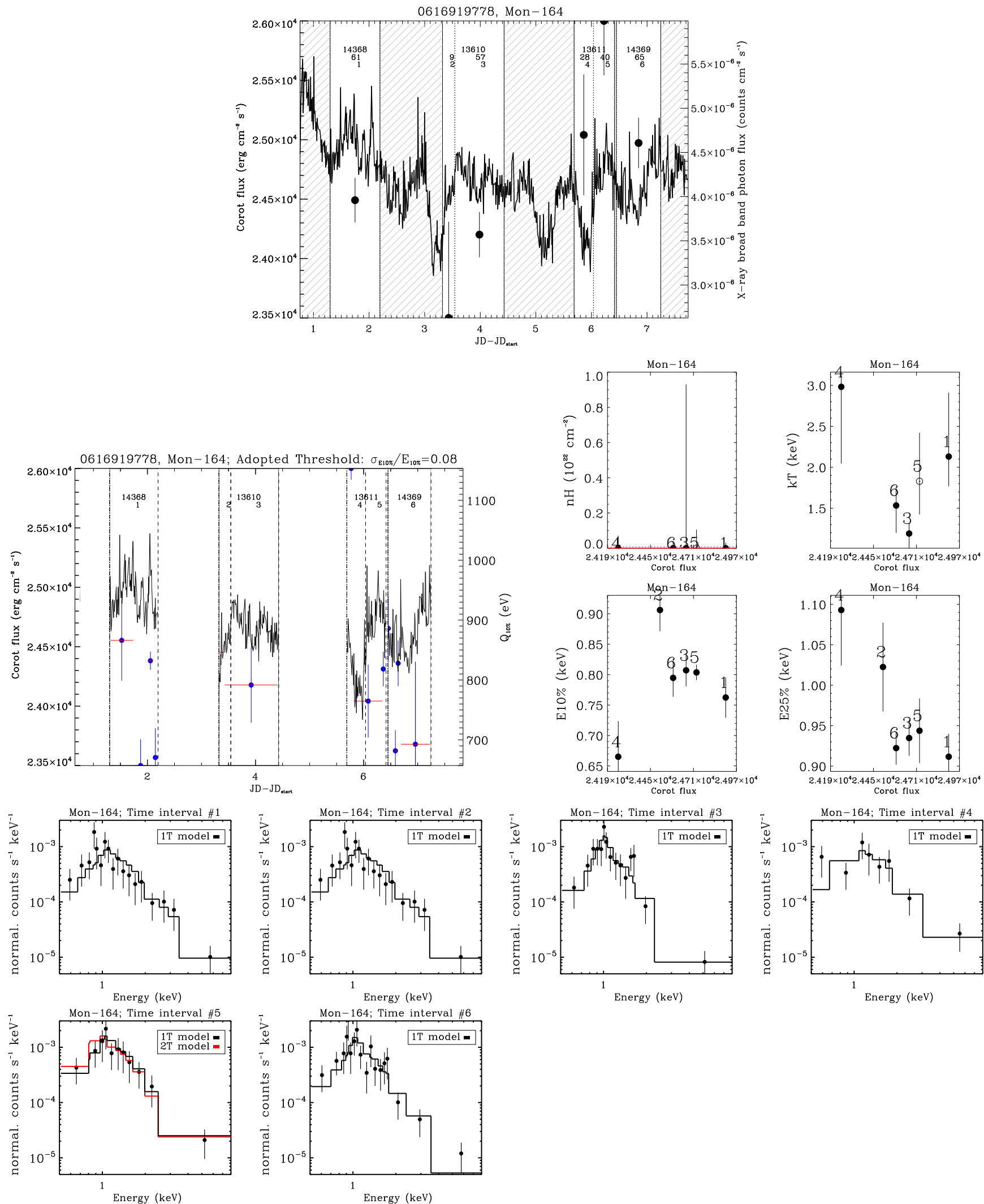

Fig. C.28. Variability and X-ray spectra of Mon-164, analyzed both as a dipper and burster, and not showing any relevant simultaneous optical and X-ray variability. 
A\&A 602, A10 (2017)
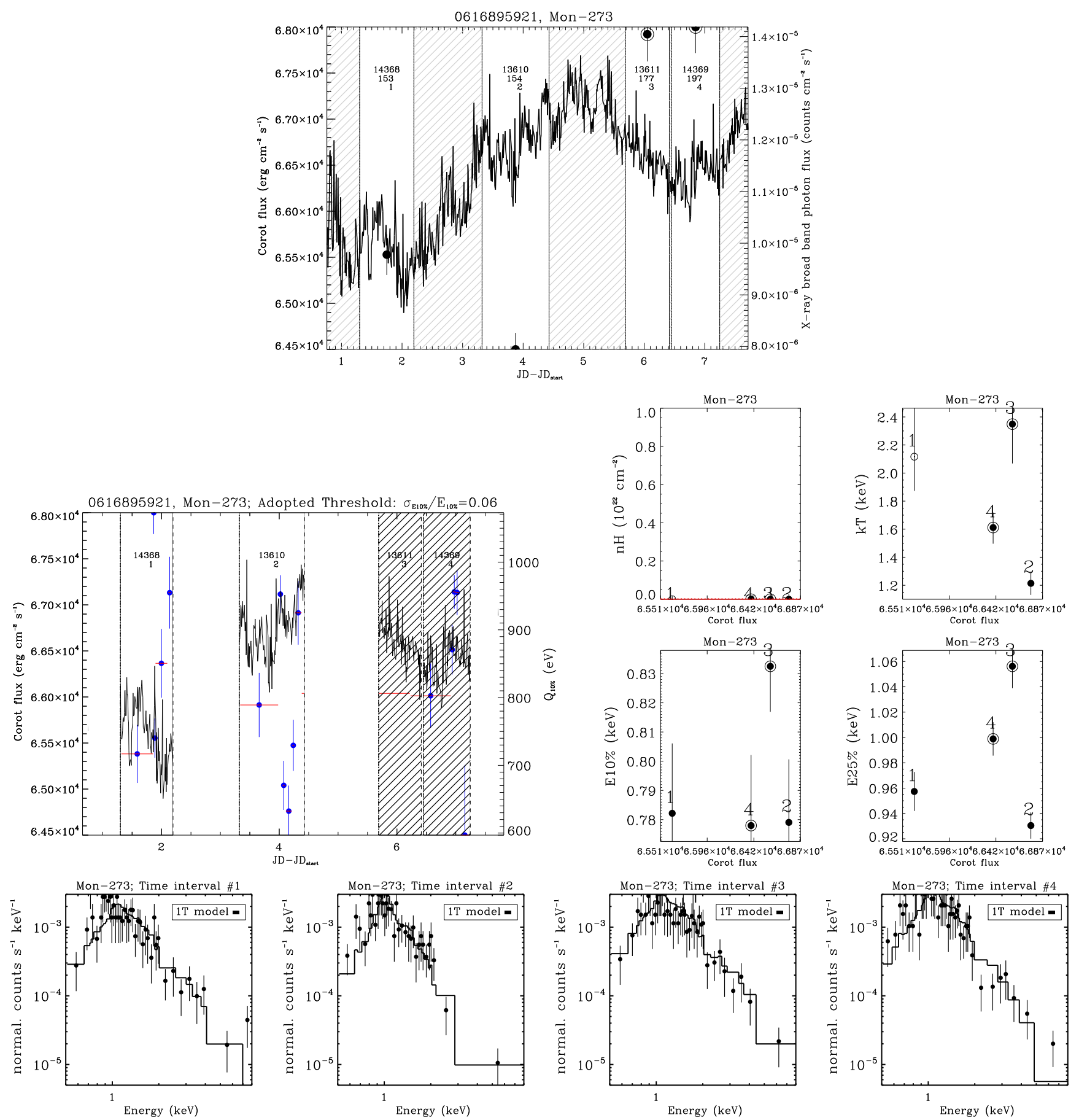

Fig. C.29. Variability and X-ray spectra of Mon-273, which have not been analyzed since the CoRoT mask around its the position is contaminated by a nearby bright source. 
M. G. Guarcello et al.: CSI 2264: Simultaneous optical and X-ray variability in pre-main sequence stars
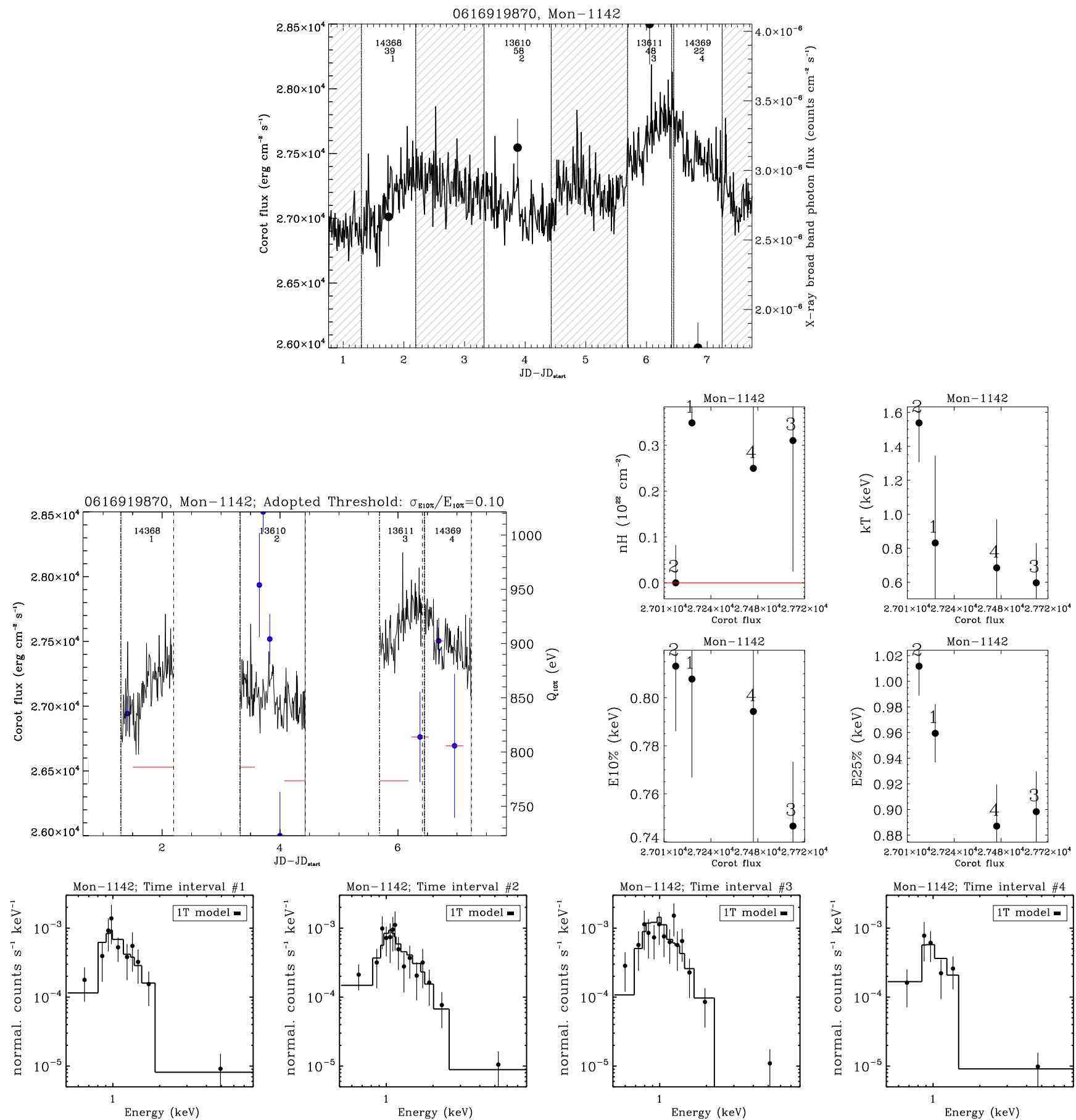

Fig. C.30. Variability and X-ray spectra of Mon-1142, analyzed both as a dipper and burster, and not showing any relevant variability. 

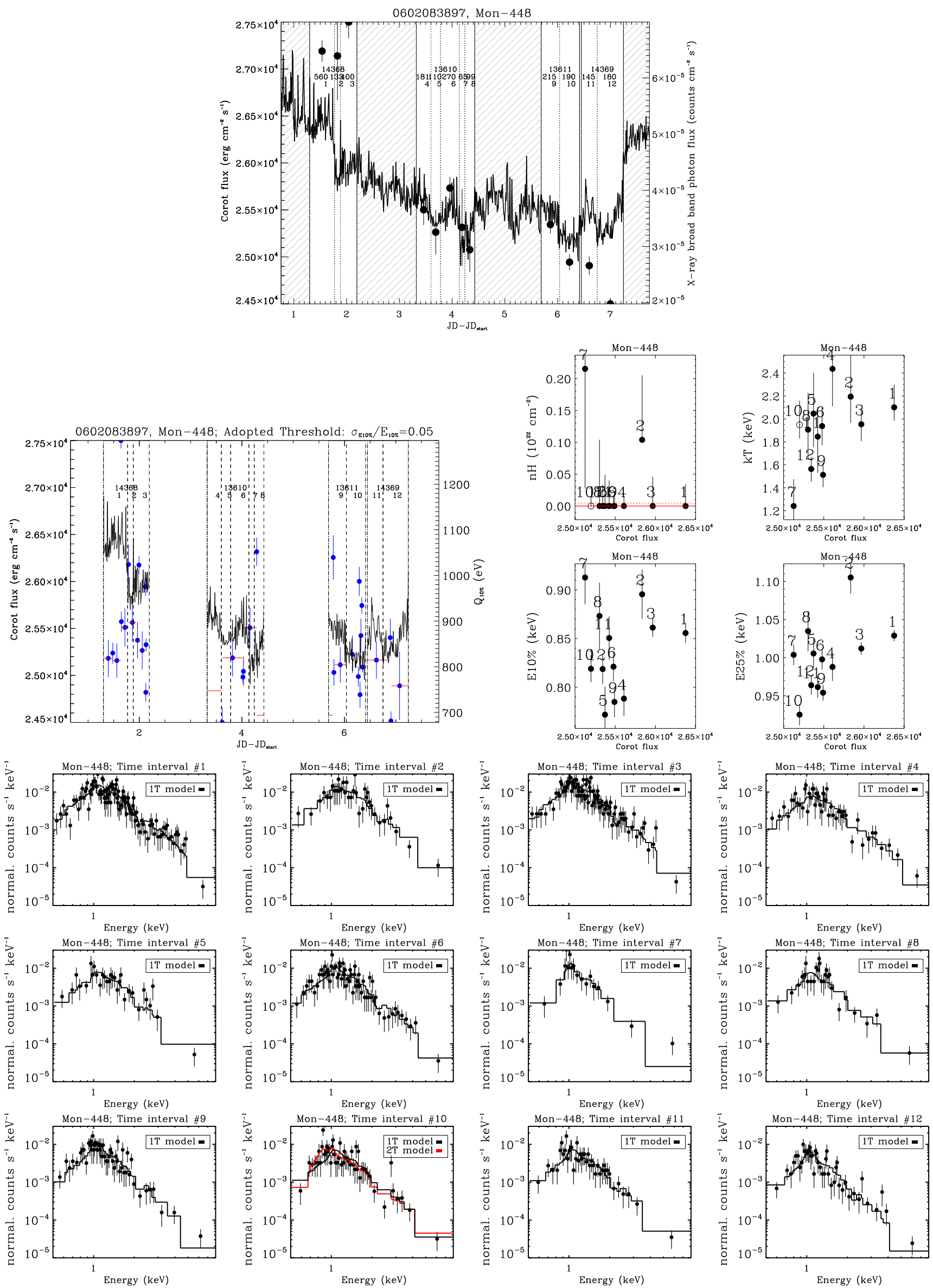

Fig. C.31. Variability and X-ray spectra of Mon-448, analyzed both as a dipper and burster. The features observed in the CoRoT light curve do not correspond to any significant variability of the $\mathrm{X}$-ray properties. 
M. G. Guarcello et al.: CSI 2264: Simultaneous optical and X-ray variability in pre-main sequence stars
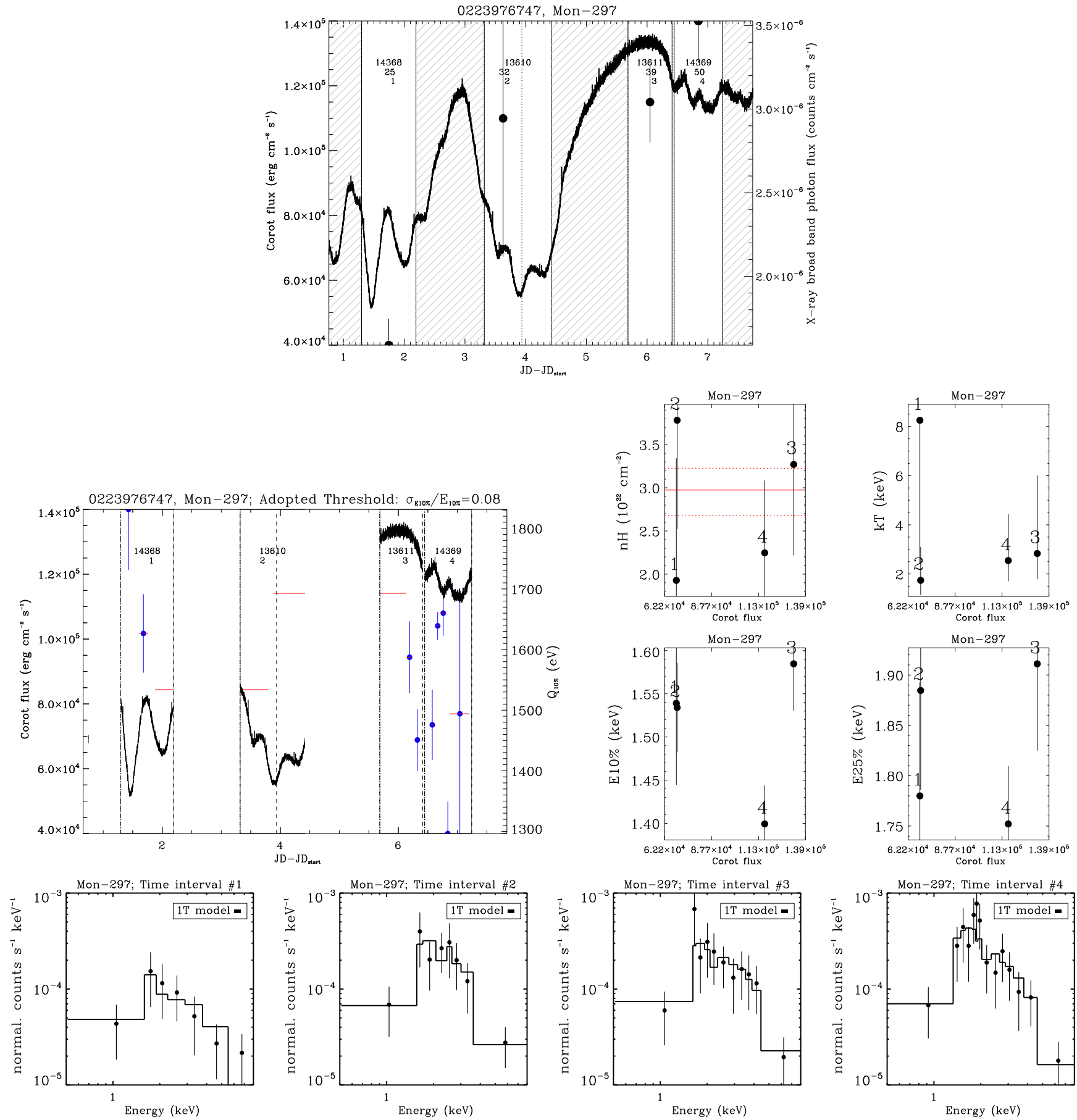

Fig. C.32. Variability and X-ray spectra of Mon-297, analyzed both as a dipper and burster. The features observed in the CoRoT light curve do not correspond to any significant variability of the X-ray properties given the few X-ray photons detected. The variability of $E_{10 \%}$ during \#1 suggests that the X-ray spectrum may be harder during the first dip. 
A\&A 602, A10 (2017)
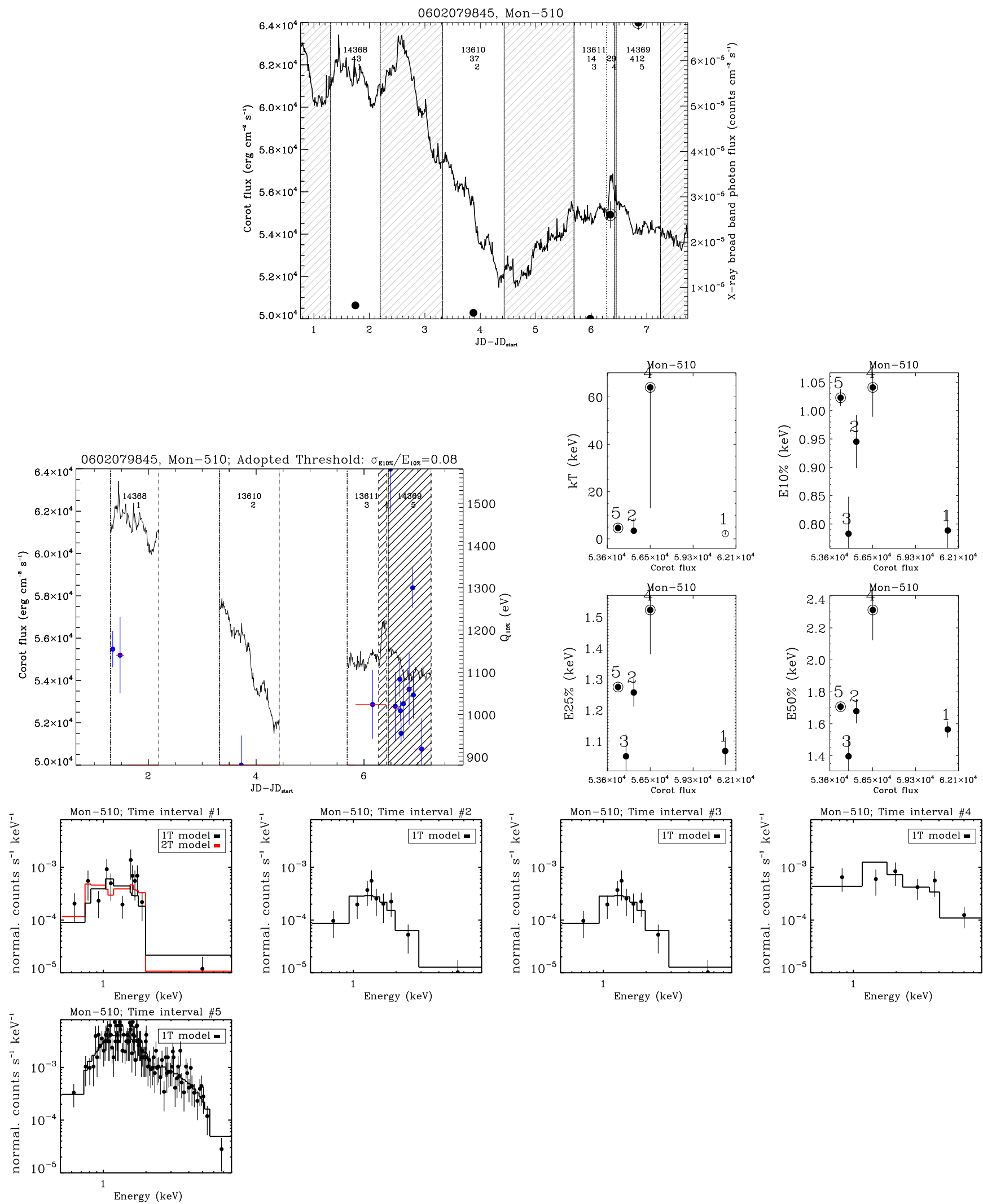

Fig. C.33. Variability and X-ray spectra of Mon-510, analyzed as a burster. The features observed in the CoRoT light curve do not correspond to any significant variability of the $\mathrm{X}$-ray properties. 
M. G. Guarcello et al.: CSI 2264: Simultaneous optical and X-ray variability in pre-main sequence stars
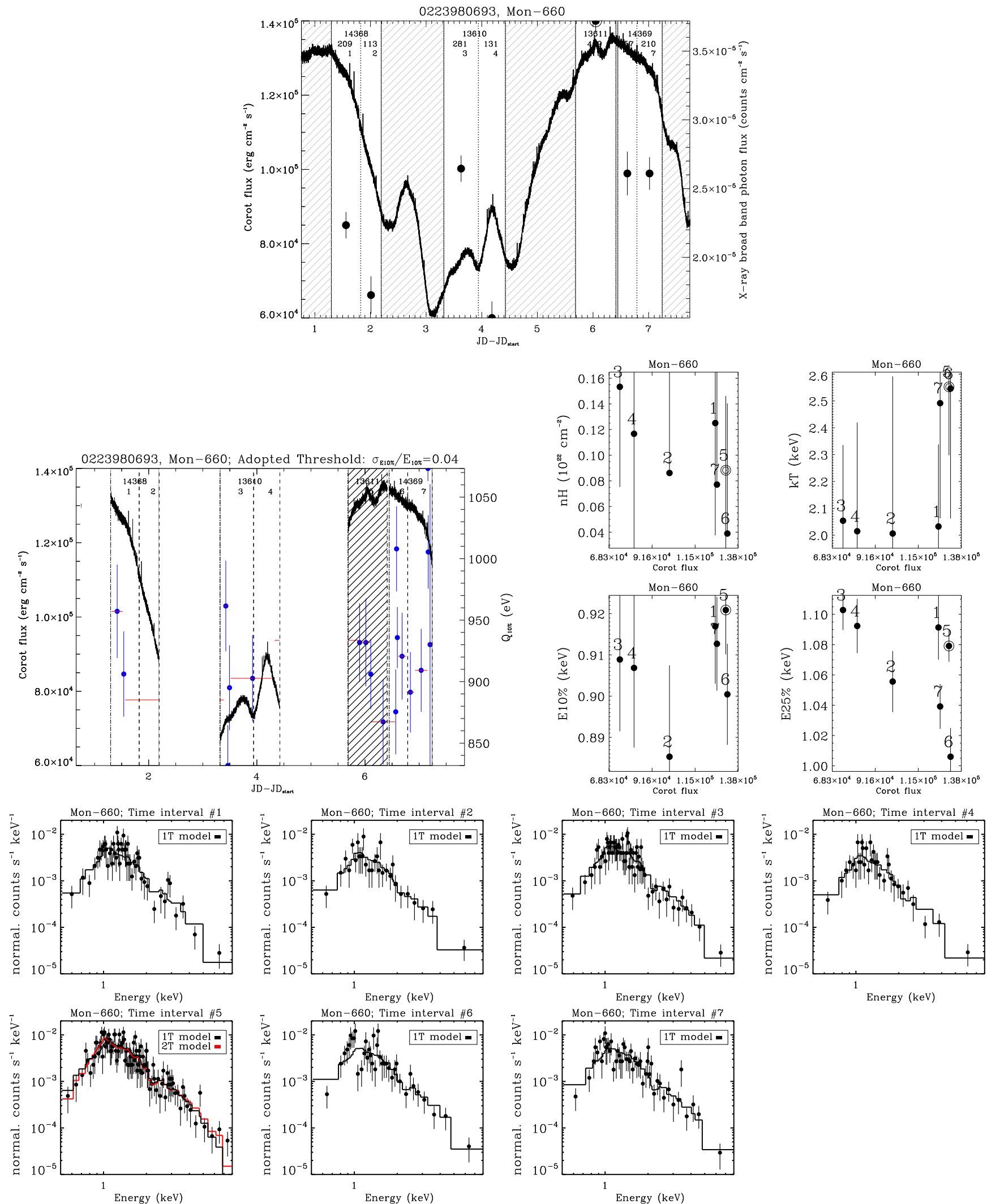

Fig. C.34. Variability and X-ray spectra of Mon-660, analyzed both as a dipper and burster. The features observed in the CoRoT light curve do not correspond to any significant variability of the $\mathrm{X}$-ray properties. 
Appendix D: Entire CoRoT light curves of the stars discussed in this paper

In this appendix, we show the whole CoRoT light curves of the stars with disks discussed in this paper. The Chandra frames are the shaded time windows delimited by vertical lines. 
M. G. Guarcello et al.: CSI 2264: Simultaneous optical and X-ray variability in pre-main sequence stars
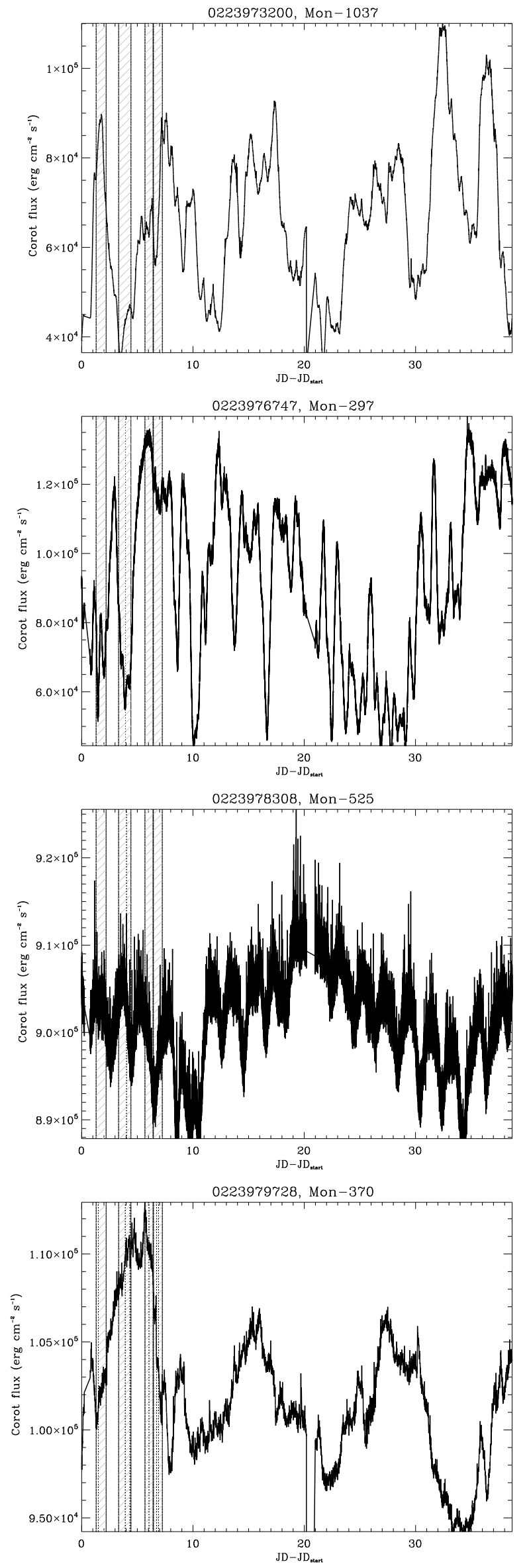
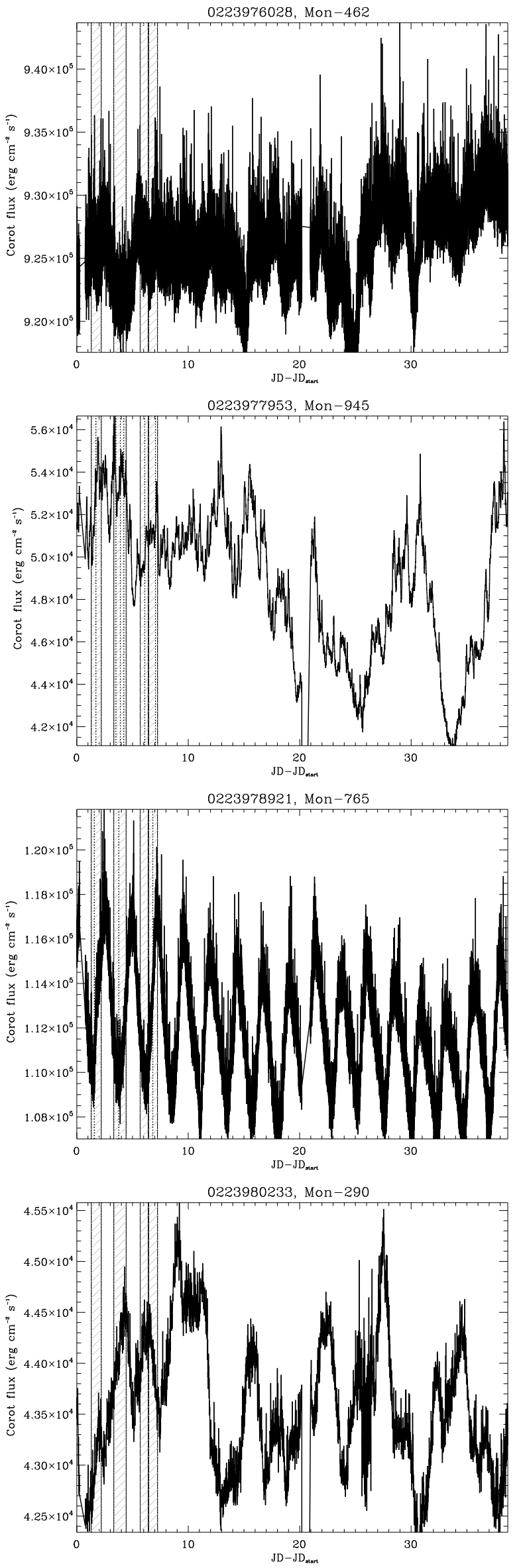

Fig. D.1. Whole CoRoT light curves of the stars discussed in the paper. 
A\&A 602, A10 (2017)
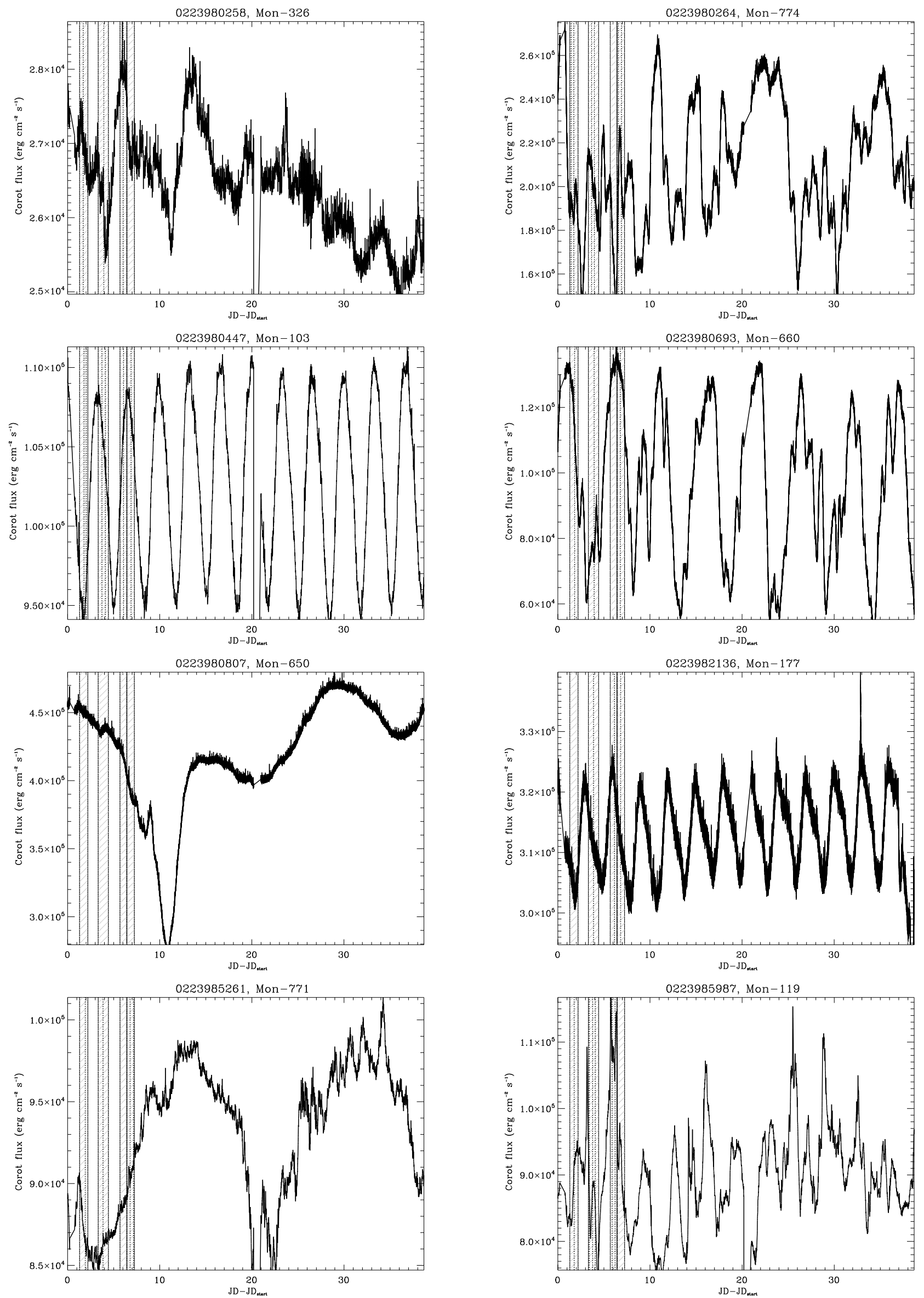

Fig. D.1. continued. 
M. G. Guarcello et al.: CSI 2264: Simultaneous optical and X-ray variability in pre-main sequence stars
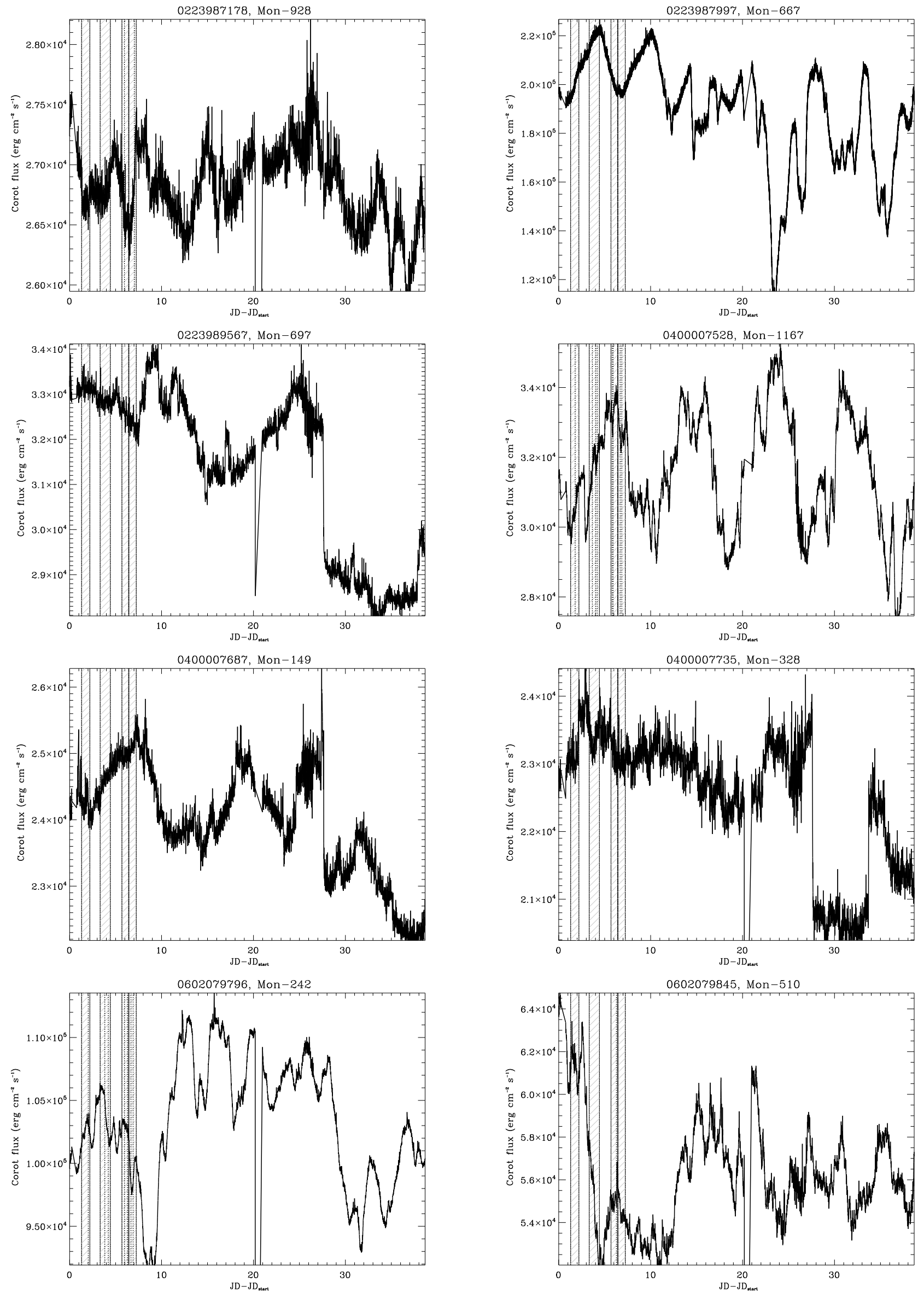

Fig. D.1. continued. 

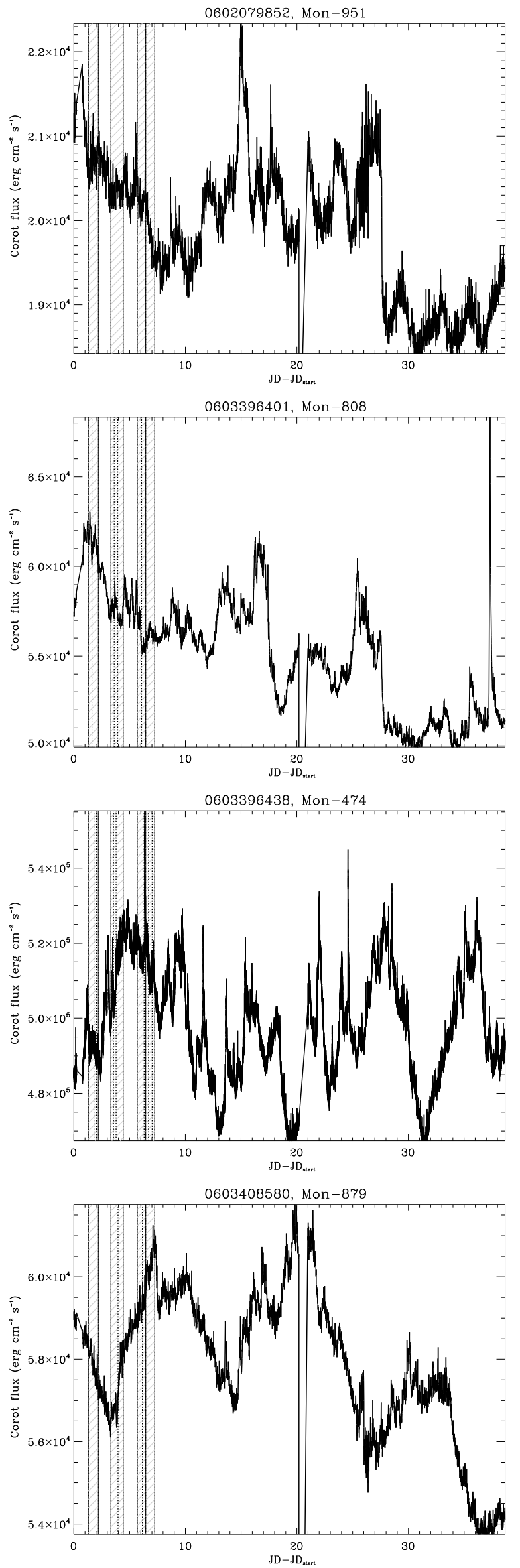
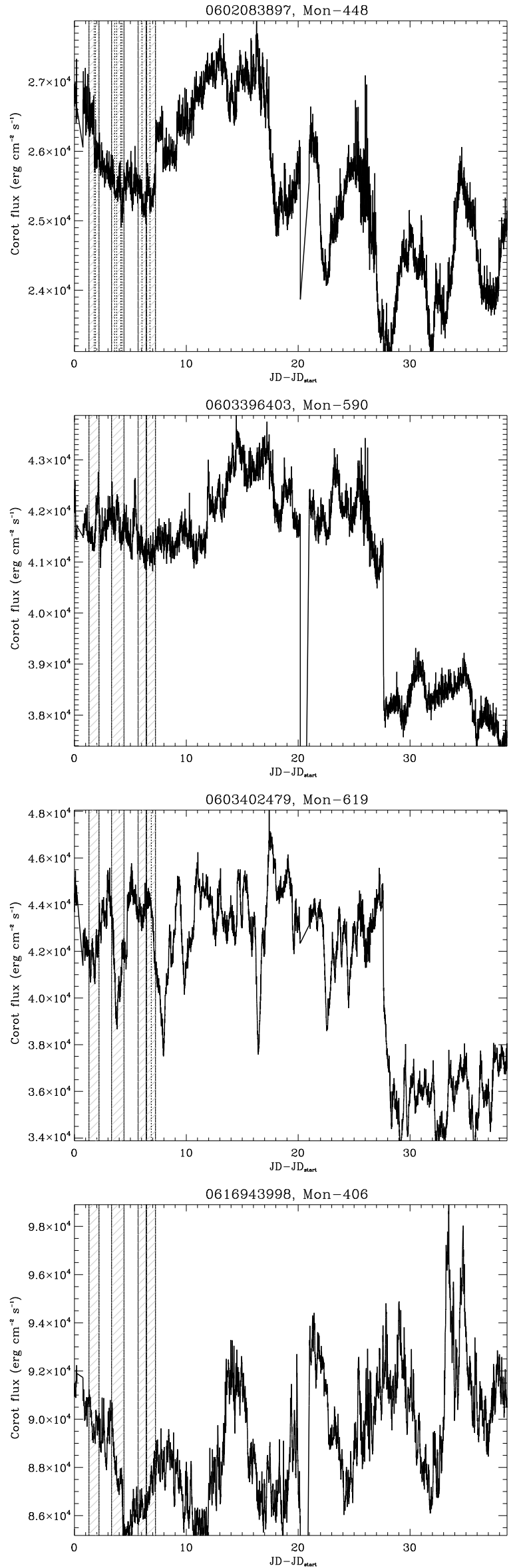

Fig. D.1. continued. 
M. G. Guarcello et al.: CSI 2264: Simultaneous optical and X-ray variability in pre-main sequence stars
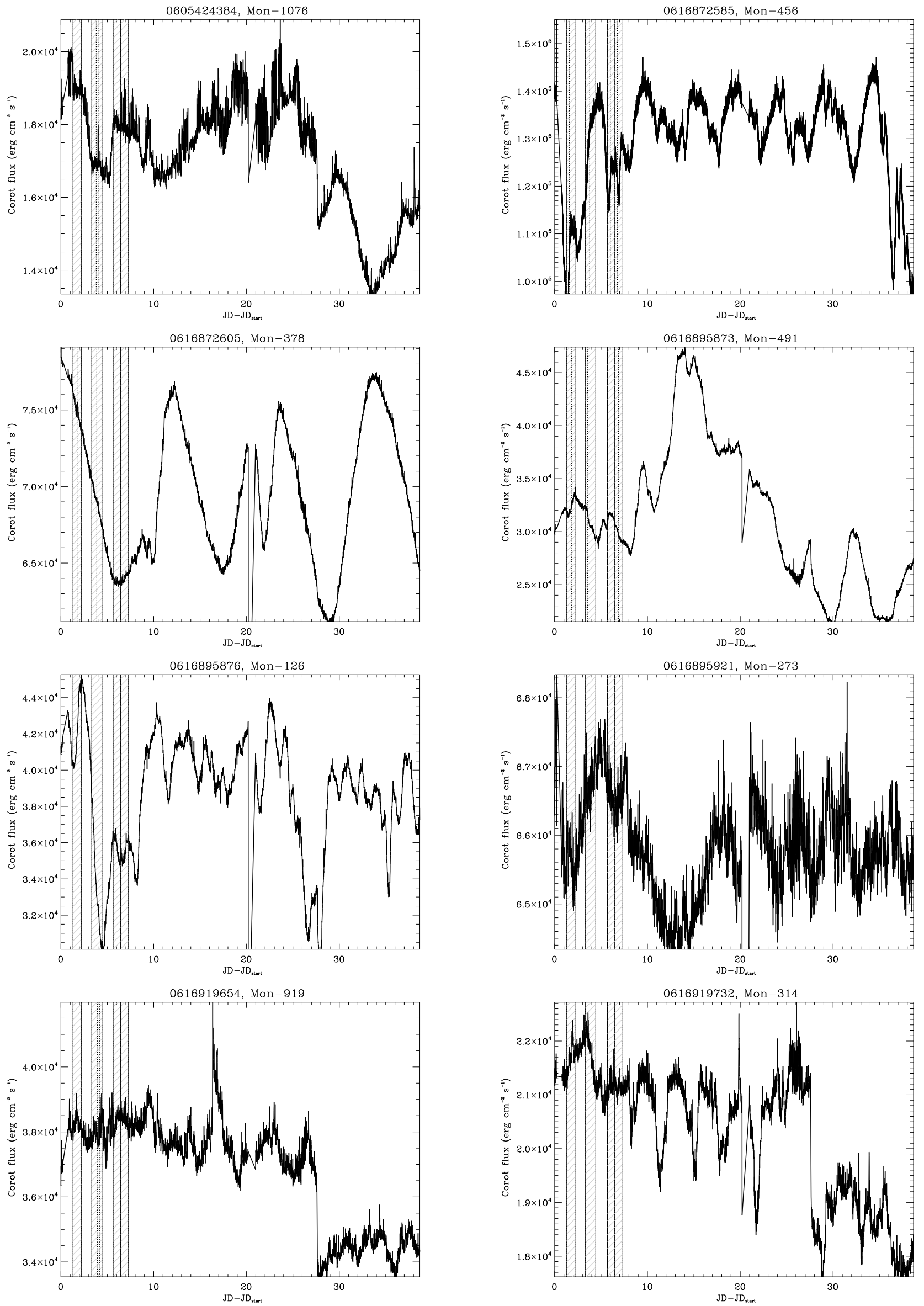

Fig. D.1. continued. 

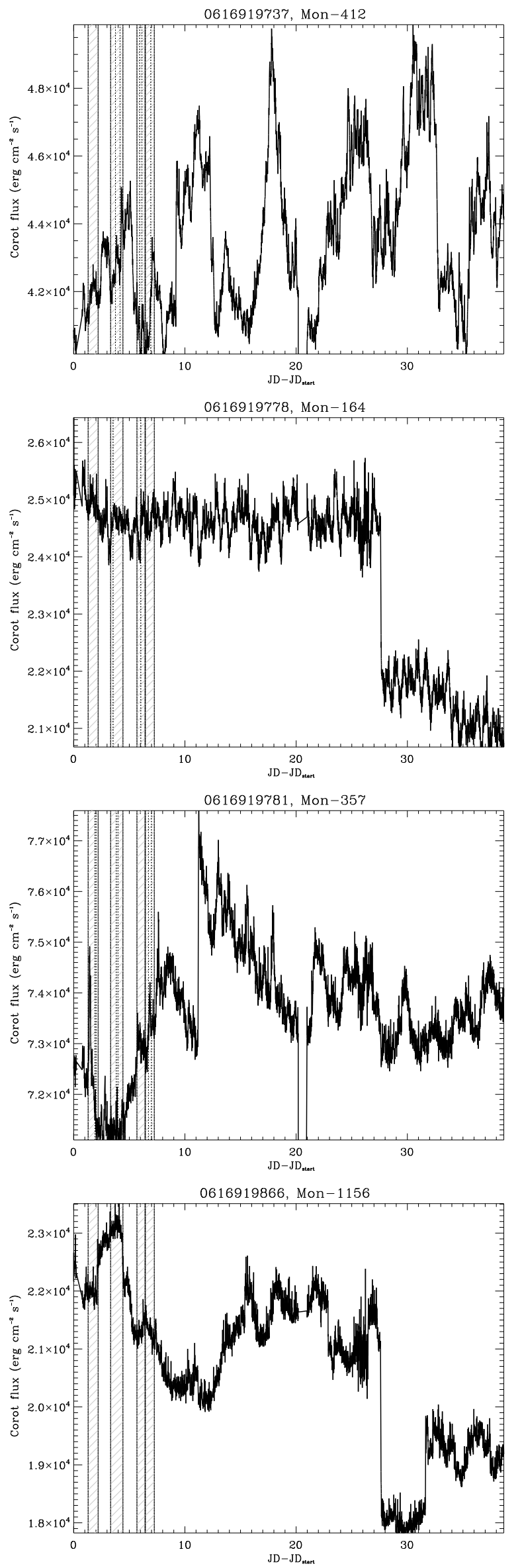
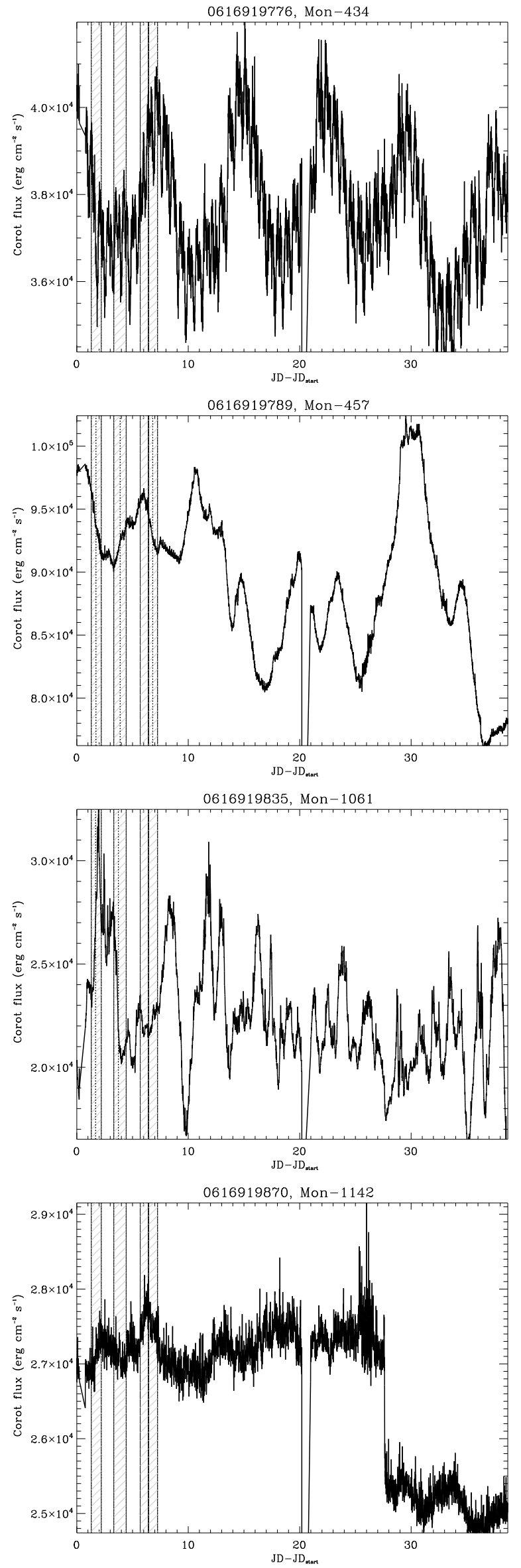

Fig. D.1. continued. 
M. G. Guarcello et al.: CSI 2264: Simultaneous optical and X-ray variability in pre-main sequence stars
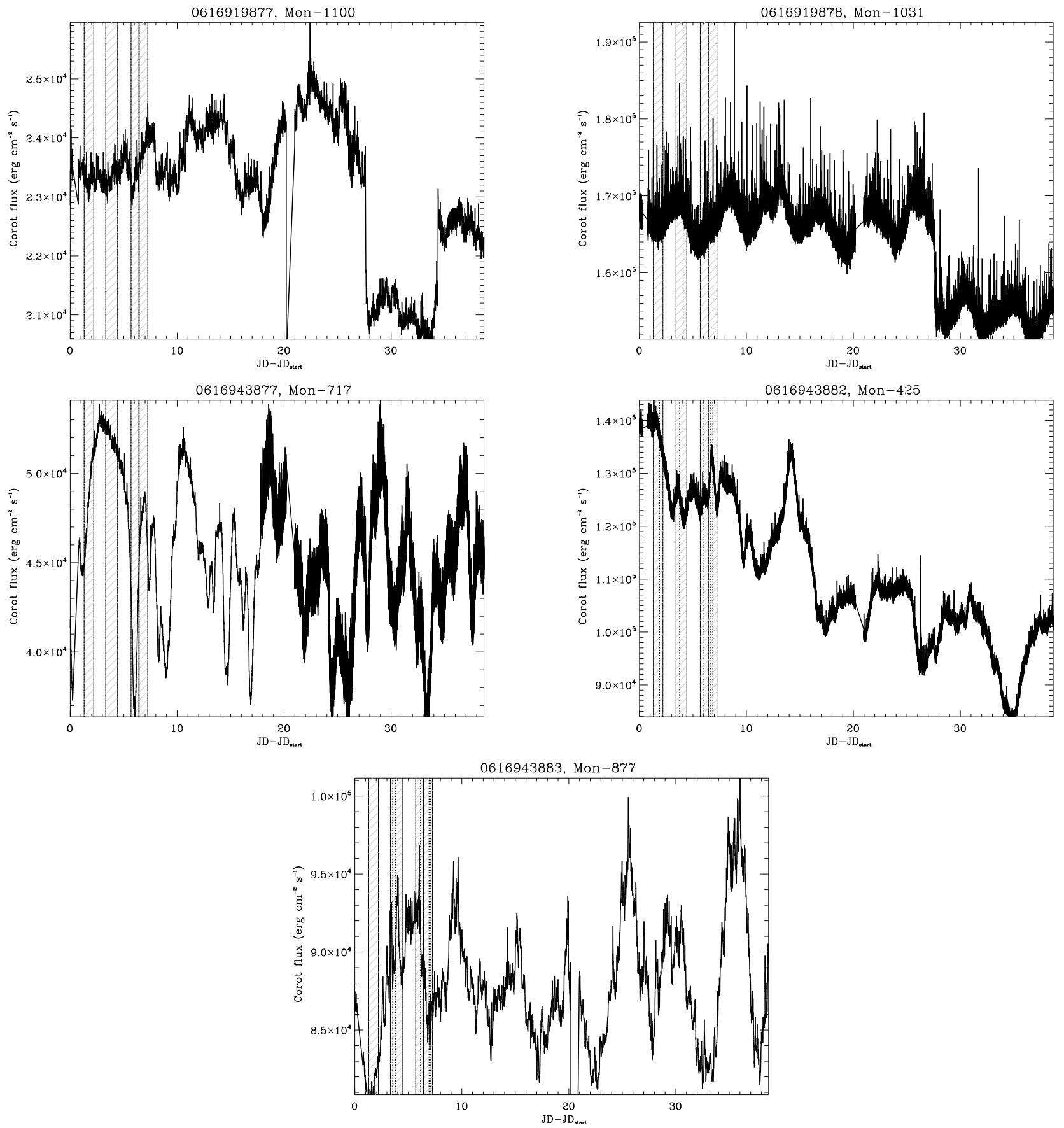

Fig. D.1. continued. 